\title{
Spinor frame formalism for amplitudes and constrained superamplitudes of 10D SYM and 11D supergravity
}

\author{
Igor Bandos \\ Department of Theoretical Physics, University of the Basque Country UPV/EHU, \\ P.O. Box 644, 48080 Bilbao, Spain \\ IKERBASQUE, Basque Foundation for Science, \\ 48011, Bilbao, Spain \\ E-mail: igor.bandos@ehu.eus
}

ABSTRACT: We show that 10D spinor helicity formalism can be understood as spinor moving frame approach to supersymmetric particles extended to the description of amplitudes. This allows us to develop the spinor helicity formalism for 11D supergravity and a new constrained superfield formalism for 10D SYM and 11D SUGRA amplitudes. We show how the constrained on-shell superfields, one-particle counterparts of the superamplitudes, can be obtained by quantization of massless superparticle mechanics.

We make some stages towards the calculation of amplitudes of 10D SYM and 11D SUGRA in this framework. In particular we have found supersymmetric Ward identities for constrained amplitudes and an especially convenient gauge, fixed on the spinor frame variables corresponding to scattered particles, which promises to be an extremely useful tool for further development of our approach.

We also discuss a candidate for generalization of the BCFW recurrent relations for the constrained tree superamplitudes, indicate and discuss a problem of dependence of the expressions obtained with it on a deformation vector, which is not fixed uniquely in higher dimensional $D>4$ cases.

KeYwords: Field Theories in Higher Dimensions, Scattering Amplitudes, Superspaces, Supergravity Models

ArXiv EPrint: 1711.00914v3 


\section{Contents}

1 Introduction $\quad 1$

1.1 Other superfield approaches to maximally supersymmetric QFTs 2

1.2 Outline of the paper 3

$2 \mathrm{D}=4$ spinor helicity formalism, (super)twistors, (super)amplitudes and superparticle mechanics

2.1 Spinor helicity formalism for $\mathrm{D}=4(\mathrm{~S}) \mathrm{YM}$ amplitudes 5

2.2 BCFW recurrent relations 6

$2.3 \mathcal{N}=4$ SYM and $\mathcal{N}=8$ SUGRA superamplitudes and generalized BCFW 8

2.4 Massless superparticle mechanics and on-shell superfields 10

$\begin{array}{lll}2.4 .1 & \text { Twistor form of the massless superparticle action } & 10\end{array}$

2.4.2 Hamiltonian mechanics in twistor formulation 10

2.4.3 Quantization and appearance of the on-shell superfields 11

2.4.4 From $\mathrm{D}=4$ to $\mathrm{D}=10$ and $\mathrm{D}=11 \quad 12$

3 Superparticle in ten and eleven dimensions. Spinor moving frame formulation

3.1 Brink-Schwarz action for massless superparticle in $D=10$ and $D=11 \quad 13$

$\begin{array}{ll}3.2 & \text { Moving frame formulation of massless superparticle mechanics }\end{array}$

$\begin{array}{lll}3.3 & \text { Properties of moving frame variables (vector harmonics) } & 14\end{array}$

$\begin{array}{lll}3.4 & \text { Spinor moving frame variables (spinor harmonics) } & 16\end{array}$

$\begin{array}{lll}\text { 3.5 Spinor moving frame formulation of the massless superparticle action } & 18\end{array}$

4 Spinorial frames, $\mathrm{D}=10$ spinor helicity formalism of Caron-Huot and $\begin{array}{ll}\text { O'Connell, and its } \mathrm{D}=\mathbf{1 1} \text { generalization } & 18\end{array}$

4.1 $\mathrm{D}=10$ and $\mathrm{D}=11$ spinor moving frame as spinor helicity variables $\quad 19$

4.2 D=10 SYM multiplet in spinor helicity formalism 21

4.3 11D SUGRA in spinor helicity formalism 22

4.4 10D amplitudes in spinor helicity formalism, supersymmetry and Ward identities 23

4.5 Supersymmetric Ward identities for 11D SUGRA amplitudes 25

$5 \mathrm{D}=10$ and $\mathrm{D}=\mathbf{1 1}$ on-shell superfields from quantization of massless superparticle in analytical basis of Lorentz harmonic superspace $\quad 25$

5.1 Changing variables in $\mathrm{D}=10,11$ massless superparticle action 25

5.2 Analitical basis of Lorenz harmonic superspace and its invariant $\begin{array}{ll}\text { sub-superspaces } & 26\end{array}$

5.3 Cartan forms and derivatives of the spinor moving frame variables 28

$\begin{array}{ll}\text { 5.3.1 Cartan forms and vector harmonics } & 28\end{array}$

$\begin{array}{ll}\text { 5.3.2 Derivatives of spinorial harmonics } & 29\end{array}$ 
5.4 Massless superparticle action in the analytical basis of Lorentz harmonic superspace 30

5.5 Massless $\mathrm{D}=10$ and $\mathrm{D}=11$ superparticle quantization in the analytical basis 30

5.5.1 On-shell superspaces for 10D SYM and 11D SUGRA 33

5.5.2 On-shell superfields and superfield equations of 10D SYM 34

5.5.3 On-shell SUSY and supersymmetric invariant of the (linearized) 10D SYM

5.5.4 On-shell superfields, superfield equations and supersymmetric invariant of linearized 11D SUGRA

6 Superfield representation for 10D SYM and 11D SUGRA amplitudes 37

6.1 Superfield representation of the 10D SYM amplitudes: 10D superamplitudes 37

6.2 Tree superamplitudes in $\mathrm{D}=11 \quad 40$

6.3 Generalization of the BCFW deformations for 10D and 11D superamplitudes 42

6.3.1 Bosonic part of $\mathrm{D}=10 \mathrm{BCFW}$ deformation and its $11 \mathrm{D}$ generalization 42

6.3.2 Fermionic part of the $\mathrm{D}=10$ and $\mathrm{D}=11 \mathrm{BCFW}$-type deformations 44

6.4 Candidate BCFW recurrent relations for 10D SYM superamplitudes 45

6.5 On validity of higher dimensional BCFW relations 46

6.6 Candidate BCFW recurrent relation for the 11D supergravity amplitudes 47

7 Towards calculation of $11 \mathrm{D}$ and $10 \mathrm{D}$ amplitudes

7.1 Candidate BCFW-type relation for 4-point superamplitudes in $\mathrm{D}=11 \quad 49$

7.2 On 4-particle and 3-particle amplitudes in $\mathrm{D}=11 \quad 50$

7.3 Relation between spinor frames associated to different external particles $\quad 53$

7.4 Reference spinor frame and complete gauge fixing of the auxiliary gauge symmetries $\quad 55$

7.5 Momentum conservation and Mandelstam variables 56

7.6 Studying the BCFW-like deformation with the gauge fixed spinor frames $\quad 57$

7.73 - and 4- particle kinematics. Momentum conservation 59

$\begin{array}{lll}7.8 & t_{3} \text { tensor } & 61\end{array}$

$\begin{array}{lll}7.9 & \text { Supermomentum } & 61\end{array}$

7.9.1 Invariants from the projections of supermomentum 63

7.9.2 Invariants from the complete spinorial supermomentum 63

8 Studying the candidate BCFW-type recurrent relations for 4-particle amplitudes of 10D SYM

8.1 Candidate BCFW for 4-fermionic amplitude in 10D SYM from super-BCFW for 4-point superamplitude

8.2 3-point amplitudes with two fermionic particles in 10D SYM

8.3 Testing the candidate BCFW by calculating 4-gluino amplitude.

Problem of dependence on deformation vector

8.4 From the candidate BCFW relation to an expression for 10D SYM amplitude with two bosonic and two fermionic legs. Deformation vector dependence 


\section{A Clifford superfield version of $D=10$ superamplitudes and fermionic BCFW deformation}

A.1 SUSY generator representation with Clifford algebra element 72

A.2 Clifford superfield version of $\mathrm{D}=10 \mathrm{BCFW}$ deformation 73

\section{Introduction}

The seminal paper [1] initiated a wave of renewed interest to the applications of $\mathrm{D}=4$ twistor approach [2-8] which resulted in development of new methods of computation of on-shell amplitudes and (in combination with other ideas [9]) to a significant progress in loop calculations [9-15]. Of these new methods let us mention the Britto-Cachazo-Feng-Witten (BCFW) approach [16], which allowed to obtain Britto-Cachazo-Feng (BCF) recursion relations for tree amplitudes [17] and their loop generalizations. It was further developed and generalized for superamplitudes of maximally supersymmetric $4 \mathrm{D} \mathcal{N}=4$ super-YangMills (SYM) and $\mathcal{N}=8$ supergravity (SUGRA) theories in [18-22] and refs. therein.

To be precise, the original BCFW work [16] as well as [18-22] used essentially the socalled $(D=4)$ spinor helicity formalism, ${ }^{1}$ which is related but not identical to the above mentioned twistor approach. ${ }^{2} \mathrm{~A} \mathrm{D}=10$ dimensional generalization of the spinor helicity formalism was constructed by Caron-Huot and O'Connell in [25] and used in [26-29] to study the 10D SYM and type IIB supergravity amplitudes (see [27] also for application to type IIB string theory amplitudes). The authors of [25] also constructed a superfield generalization of 10D amplitudes, which we have called 'Clifford superamplitude' in [30]. However this formalism happens to be quite nonminimal and very complicated, so that the further use of 10D spinor helicity formalism was in the field of type IIB supergravity where an essential simplification can be reached with the use of the natural IIB complex structure.

In this paper we first show that the variables of the 10D spinor helicity formalism can be identified with spinor moving frame variables (Lorentz harmonics) used for the description of supersymmetric particle mechanics in [31-33]. ${ }^{3}$ The spinor moving frame formalism was also developed for the case of 11D massless superparticle $[35-38]^{4}$ and we

\footnotetext{
${ }^{1}$ As it was shown in [19], the bosonic BCFW relation can be also proved without the use of spinor helicity variables; this fact does not diminish the significance of these which were used quite extensively in [19].

${ }^{2}$ Also a momentum twistor formalism, alternative (dual) to the standard twistor approach and related to dual superconformal symmetry [10], rather than to the standard conformal symmetry of $\mathcal{N}=4 \mathrm{SYM}$, was developed in [23, 24].

${ }^{3}$ The identification of spinor helicity variables with Lorentz harmonics were noticed in [34] and used there to construct $\mathrm{D}=5$ spinor helicity formalism.

${ }^{4}$ The spinor moving frame approach for $\mathrm{D}=4$ and $\mathrm{D}=10$ superstrings was proposed in [39, 40] and elaborated in [41], for 11D supermembrane in [42, 43] and for the generic super-p-branes from the 'standard brane scan' - in [44]. The synthesis of spinor moving frame approach with the so-called STV (Sorokin-TkachVolkov) approach to superparticles and superstrings [45-47] (see [48] for the review and more references) resulted in the development of the superembedding approach to superstrings and super-p-branes [49]. In particular, in the frame of this approach (also reviewed in [48]) the equations of motion of the M-theory 5-brane had been obtained in [50] some months before the covariant actions was constructed in [51] and [52].
} 
have used it to develop the spinor helicity formalism for 11D supergravity amplitudes. This has been briefly reported in [30] and is elaborated here.

A key observation is that a suitable quantization of the $\mathrm{D}=4 \mathcal{N}=4$ and $\mathcal{N}=8$ superparticle models in its twistor-like formulation [6,7] results in description of their quantum state spectrum by so-called on-shell superfields. These are chiral superfields in superspace with $\mathcal{N}$ complex fermionic and 2 complex $(4$ real) bosonic spinor coordinates, known as on-shell superspace, which describe the fields of the linearized $\mathcal{N}=4 \mathrm{SYM}$ and $\mathcal{N}=8$ SUGRA multiplets (see e.g. $[1,10]$ ). The superamplitudes of $\mathcal{N}=4 \mathrm{SYM}$ and $\mathcal{N}=8$ can be considered as multiparticle generalizations of these on-shell superfields.

Similarly, a suitable quantization of the 10D and 11D massless superparticle models in their spinor moving frame formulations [33, 36-38] results in an on-shell superfield description of the linearized 10D SYM and 11D SUGRA supermultiplets which had been proposed in [35] (as a generalization of the Penrose twistor transform [2-5]). As, in distinction to the $4 \mathrm{D}$ case, these on-shell superfields obey some nontrivial differential equations with fermionic covariant derivatives, we call them constrained on-shell superfields. Multiparticle generalizations of these give us constrained superamplitudes which are much more economic than the Clifford superamplitudes of [25]. The development of such a constrained superamplitude formalism for 10D SYM and 11D SUGRA is one of the main aims of the present paper.

We describe the properties of the constrained tree superamplitudes of 10D SYM and 11D SUGRA and make some stages necessary for practical calculations of amplitudes in the frame of our spinor helicity formalism and constrained superamplitude approach. In particular we obtain the supersymmetric Ward identites and present a convenient gauge fixed on the spinor frame variables, which can be considered as Lorentz covariant counterpart of the light-cone gauge.

We have also presented a candidate for generalization of the BCFW recurrent relations for the constrained tree superamplitudes and studied its properties. In this part the results are quite preliminary. The use of our candidate BCFW relations to calculate 4-point SYM amplitudes leads to expressions suffering an unwanted explicit dependence on a deformation vector, which is not fixed uniquely in the case of higher $D$. We suggest that this can be improved with the use of a complementary analytic superamplitude formalism and complex spinor frame variables of [53].

A part of the 11D results was briefly reported in [30]. An alternative (although related) analytic superamplitude formalism has been proposed in [53]. The use of this latter for further development of the constrained superamplitude formalism of the present paper will be the subject of future publication.

\subsection{Other superfield approaches to maximally supersymmetric QFTs}

We have to notice the existence of other superfield approaches to calculation of the amplitudes which are oriented on the use of the off-shell methods, actions and Feynman diagrams.

First of all there is the light-cone superspace approach used in particular to prove the perturbative finiteness of the $\mathcal{N}=4$ SYM theory in $\mathrm{D}=4$ [54-56]. It was also developed 
for superstring theory [57]. Despite it was proved to be very useful, the lack of an explicit Lorentz covariance was the reason to search for manifestly Lorentz covariant formalism.

The covariant superfield approach of [58], which was based on the off-shell supersymmetry and background superfield methods, allowed to obtain the non-renormalization theorems and analyse the structure of possible counterterms. It brought a number of impressive results, in particular an alternative proof of perturbative finiteness of $\mathcal{N}=4 \mathrm{SYM}[58]$ and of the absence of some loop divergences in $\mathcal{N}=8$ supergravity [59]. The restriction of this method comes from the need in an off-shell superfield description of the supersymmetric theory, which is unknown in its complete form for the cases of most interesting higher $\mathcal{N}$ and higher dimensional supergravities and SYM theories. ${ }^{5}$

The analysis of the on-shell counterterms of $\mathcal{N}$-extended supergravity theories based on their on-shell superfield description was initiated in [60,61] and is still in the center of interest (see e.g. [13] and refs. therein).

The so-called pure spinor approach to superstring [62-65] was also developed for supersymmetric field theories [66-69]. In particular, a pure spinor formulation of the action for 11D supergravity was proposed in [70], elaborated in [71, 72] and applied to analysis of UV divergences in [73].

This is a good place to notice by pass that the study of the relation of pure spinor description of superstring with the standard Green-Schwarz formulation resulted in proposing a number of versions of multidimensional twistor correspondences [74-76] alternative to the Lorentz harmonic version of twistor-like approach used in this paper and e.g. in [33, 36].

As we have already noticed, the main difference of the approach of this paper with the above mentioned superfield approaches to maximally supersymmetric quantum theories is that these use off-shell methods, Lagrangians and Feynman rules, while our study belongs to the line of the on-shell approaches to superamplitudes. The experience gained in $\mathrm{D}=4[14,15]$ suggests that the on-shell methods at the end might happen to be more practical in quantum calculations. Of course, we are at the beginning of the way and presently cannot show such impressive results of our approach as the ones obtained with the above methods.

\subsection{Outline of the paper}

The rest of the paper is organized as follows. In the next section 2 we first review the $\mathrm{D}=4$ spinor helicity formalism (section 2.1) and $\mathrm{BCFW}$ recurrent relations for $\mathrm{D}=4 \mathrm{am}$ plitudes (section 2.2), as well as the on-shell superspace, $\mathrm{D}=4$ superamplitudes and their one-particle counterparts called 'on-shell superfields' (section 2.3). In section 2.4. we show how these on-shell superfields can be obtained by quantization of $\mathcal{N}$-extended massless superparticle model.

This $\mathrm{D}=4$ observation provides us with a guide in search for on-shell superamplitudes of higher dimensional SYM and SUGRA theories. Namely, first we have to find a suitable superparticle model and perform its quantization in a suitable representation thus arriving

\footnotetext{
${ }^{5}$ The result on finiteness of $\mathcal{N}=4 \mathrm{SYM}$ were obtained in [58] using its description in terms of $\mathcal{N}=2$ superfields.
} 
at on-shell superfields describing the superparticle quantum state vectors. The tree superamplitudes will be multiparticle counterparts of such on-shell superfields. In particular they carry the same indices/charges/weights as on-shell superfields, but multiplicated (one for each scattered particles), and obey the set of equations which repeat the equations satisfied by on-shell superfield in variables corresponding to each of the scattered particles.

Spinor moving frame formulation of $\mathrm{D}=10$ and $\mathrm{D}=11$ massless superparticle mechanics (also known under the name 'twistor-like Lorentz harmonic formulation') is reviewed in section 3. Its quantization is described in section 5 where it is shown how the constrained on-shell superfields and more complicated (and quite non-minimal) Clifford superfields appear in this way. But before that, in section 4 we construct the $\mathrm{D}=10$ and $\mathrm{D}=11$ spinor helicity formalism by identifying the spinor helicity variables of scattered particles with the counterparts of spinor moving frame variables (Lorentz harmonics) of the massless superparticle mechanics.

In section 4 we also show how the solutions of linearized equations of motion of $10 \mathrm{D}$ SYM and 11D supergravity can written in terms of these spinor frame and related vector frame variables, and write supersymmetry transformations mixing the solutions of bosonic and fermionic equations. We also introduce there the spinor helicity representation of the amplitudes of 10D SYM and 11D SUGRA and obtain supersymmetric Ward identities for these amplitudes.

In section 5, by quantizing 10D and 11D superparticle models, we arrive at the on-shell superfield description of the linearized 10D SYM and 11D SUGRA which are generalized to the constrained superamplitudes in section 6. The quantization, described in section 5.5, requires some technical details on spinor moving frame variables and coordinate basis of Lorentz-harmonic superspace. These have been presented in sections 5.1-5.4 and are not used in the forthcoming sections. The reader not interested in quantization procedure, nor in details on properties of spinor moving frame in $\mathrm{D}=10$ and $\mathrm{D}=11$, might skip the first subsections and move directly to sub-subsection 5.5.2 using the previous sub-section 5.5.1 just for notation.

In section 6 we develop the constrained superamplitude formalism for 10D SYM and 11D SUGRA and present a candidate for BCFW type recurrent relations for these superamplitudes. The explicit form of these candidate BCFW relations for 4-point 11D superamplitude is discussed in section 7 where we also describe some further development of spinor frame approach to amplitudes and superamplitudes. In particular, we discuss there the relation between spinor frames associated to different particles, consequences of the momentum conservation, $\mathrm{D}=10,11$ supermomentum, and a gauge fixing conditions for the auxiliary gauge symmetry. These conditions describe a Lorentz harmonic counterpart of the light cone gauge, which is fixed on spinor frame variables and promises to be especially convenient for the amplitude calculations.

Finally, in section 8 we discuss the candidate BCFW relation for 10D four-point superamplitudes, reduce them to the relation for 4-point amplitudes, present the explicit expressions for 3-point amplitudes and use these to calculate 10D 4-point amplitudes with 4 and 2 fermionic legs. We find that, unfortunately, the resulting expressions for four point amplitudes suffer an unwanted dependence on a deformation vector and suggest that this 
might be improved with the use of analytic superamplitude formalism and complex spinor frame of [53].

We conclude in section 9 .

In appendix A we describe some aspects of the Clifford superfield version of the 10D superamplitude formalism of [25].

\section{$2 \mathrm{D}=4$ spinor helicity formalism, (super)twistors, (super)amplitudes and superparticle mechanics}

In this section we first review the $\mathrm{D}=4$ spinor helicity formalism for gauge theory amplitudes, BCFW recurrent relations for these amplitudes, superamplitudes of maximally supersymmetric $\mathrm{D}=4 \mathrm{SYM}$ and SUGRA theory and their one-particle counterparts called on-shell superfields. Then we show how such on-shell superfield appears as a representation of quantum state vector in quantization of a suitable formulation of massless superparticle mechanics. This provides us with a guide in search for tree on-shell superamplitudes of 10D SYM and 11D SUGRA which will be the subject of sections 5 and 6 .

\subsection{Spinor helicity formalism for $\mathrm{D}=4$ (S) YM amplitudes}

In spinor helicity approach to $\mathrm{D}=4$ scattered amplitudes of massless particles (see e.g. [10, $14,15]$ and refs. therein) the information on the (light-like) momentum $p_{\mu(i)}$ and on helicity of the $i$-th scattered particle is encoded in the complex 2-component bosonic spinor $\lambda_{A(i)}$. The momentum is given by the product of this and its complex conjugate $\bar{\lambda}_{\dot{A}(i)}=\left(\lambda_{A(i)}\right)^{*}$ :

$$
p_{A \dot{A}(i)}:=p_{\mu(i)} \sigma_{A \dot{A}}^{\mu}=2 \lambda_{A(i)} \bar{\lambda}_{\dot{A}(i)} \Leftrightarrow p_{\mu(i)}=\lambda_{(i)} \sigma_{\mu} \bar{\lambda}_{(i)}, \quad\left\{\begin{array}{l}
A=1,2, \quad \dot{A}=1,2, \\
\mu=0, \ldots, 3,
\end{array}\right.
$$

where $\sigma_{A \dot{A}}^{\mu}$ are relativistic Pauli matrices. The polarization vectors of $i$-th scattering spin 1 particles ('gluons') of negative and positive helicity can be written as

$$
\varepsilon_{A \dot{A}(i)}^{(+)}=\frac{\lambda_{A(i)} \bar{\mu}_{\dot{A}}}{\left[\bar{\lambda}_{(i)} \bar{\mu}\right]}, \quad \varepsilon_{A \dot{A}(i)}^{(-)}=\frac{\mu_{A} \bar{\lambda}_{\dot{A}(i)}}{<\mu \lambda_{(i)}>},
$$

where $\bar{\mu}_{\dot{A}}=\left(\mu_{A}\right)^{*}$ is a (constant) reference spinor, $<\mu \lambda_{(i)}>:=\epsilon_{A B} \mu^{A} \lambda_{(i)}^{B},\left[\bar{\mu} \bar{\lambda}_{(i)}\right]=$ $\epsilon_{\dot{A} \dot{B}} \bar{\mu}^{\dot{A}} \bar{\lambda}^{\dot{B}}(i)$, and

$$
\epsilon_{A B}=\left(\begin{array}{cc}
0 & -1 \\
1 & 0
\end{array}\right)=-\epsilon^{A B}, \quad \epsilon_{\dot{A} \dot{B}}=\left(\begin{array}{cc}
0 & -1 \\
1 & 0
\end{array}\right)=-\epsilon^{\dot{A} \dot{B}}
$$

are unit antisymmetric spin tensors used to lower and to rise the Weyl spinor indices,

$$
\lambda^{A}=\epsilon^{A B} \lambda_{B}, \quad \lambda_{A}=\epsilon_{A B} \lambda^{B}, \quad \bar{\lambda}_{\dot{A}}=\epsilon_{\dot{A} \dot{B}} \bar{\lambda}^{\dot{B}} .
$$

Below we will use even shorter notation for the contraction of the 'momentum spinors' or spinor helicity variables,

$$
\begin{array}{r}
<i j>\equiv<\lambda_{(i)} \lambda_{(j)}>:=\lambda_{(i)}^{A} \lambda_{A(j)}=\epsilon_{A B} \lambda_{(i)}^{A} \lambda_{(j)}^{B}, \\
{[i j] \equiv\left[\bar{\lambda}_{(i)} \bar{\lambda}_{(j)}\right]=\bar{\lambda}_{(i)}^{\dot{A}} \bar{\lambda}_{\dot{A}(j)}=\epsilon_{\dot{A} \dot{B}} \bar{\lambda}_{(i)}^{\dot{A}} \bar{\lambda}_{(j)}^{\dot{B}} .}
\end{array}
$$


One easily observes that $\langle i j\rangle=-\langle j i\rangle,[i j]=-[j i]\left(\right.$ as $\left.\epsilon_{\dot{A} \dot{B}}=-\epsilon_{\dot{B} \dot{A}}(2.3)\right)$ and, hence, $<i i>=0=[i i]$. Then the light-likeness of the momentum (2.1) and its orthogonality to polarization vectors $(2.2), p_{(i)}^{\mu} \varepsilon_{\mu(i)}^{( \pm)}=0$, follow from the identity

$$
\sigma_{A \dot{A}}^{\mu} \sigma_{\mu B \dot{B}}=2 \epsilon_{A B} \epsilon_{\dot{A} \dot{B}}
$$

The scattering amplitudes $\mathcal{A}(1, \ldots, n):=\mathcal{A}\left(p_{(1)}, \varepsilon_{(1)} ; \ldots, p_{(n)}, \varepsilon_{(n)}\right)$ are independent of the choice of $\mu$ in (2.2), so that

$$
\mathcal{A}(1, \ldots, n):=\mathcal{A}\left(p_{(1)}, \varepsilon_{(1)} ; \ldots ; p_{(n)}, \varepsilon_{(n)}\right)=\mathcal{A}\left(\lambda_{(1)}, \bar{\lambda}_{(1)} ; \ldots ; \lambda_{(n)}, \bar{\lambda}_{(n)}\right) .
$$

Furthermore, as a function of bosonic spinors, the amplitude should obey the helicity constraints,

$$
\hat{h}_{(i)} \mathcal{A}(1, \ldots, n)=h_{i} \mathcal{A}(1, \ldots, n),
$$

where $h_{i}$ is the helicity of the state, $h_{i}= \pm 1$ in the case of gluons, and $\hat{h}_{(i)}$ is helicity operator which has the form

$$
\hat{h}_{(i)}:=\frac{1}{2} \lambda_{(i)}^{A} \frac{\partial}{\partial \lambda_{(i)}^{A}}-\frac{1}{2} \bar{\lambda}_{(i)}^{\dot{A}} \frac{\partial}{\partial \bar{\lambda}_{(i)}^{\dot{A}}} .
$$

Thus $n$-particle amplitude is also characterized by $n$ helicities. In the case of $n$ gluons these are \pm 1 and the amplitude carries $n$ sign indices,

$$
\mathcal{A}(1, \ldots, n)=\mathcal{A}^{-\ldots-\ldots+\ldots+}(1, \ldots, n) .
$$

It can be shown that the amplitudes with all and with all but one gluons of the same helicity vanish, i.e. $\mathcal{A}^{+\ldots+}(1, \ldots, n)=0, \mathcal{A}^{-+\ldots+}(1, \ldots, n)=0$, so that the simplest maximal helicity violation ( $M H V$ ) amplitude is

$$
\mathcal{A}^{\mathrm{MHV}}(1, \ldots, n)=\mathcal{A}^{+\ldots+-_{i}+\ldots+{ }_{j}+\ldots+}(1, \ldots, n) .
$$

This can be expressed in a simple way [77] in terms of the contractions (2.4) of the lefthanded bosonic spinors corresponding to different external particles,

$$
\delta^{4}\left(\sum_{i} p_{a(i)}\right) \mathcal{A}^{\mathrm{MHV}}(1, \ldots, n)=\delta^{4}\left(\sum_{i} \lambda_{A(i)} \bar{\lambda}_{\dot{A}(i)}\right) \frac{<i j>^{4}}{<12>\ldots<(n-1) n><n 1>} .
$$

In (2.11) $i$-th and $j$-th particles are assumed to be of negative helicity $(-1)$ (as explicitly written in $(2.10))$.

\subsection{BCFW recurrent relations}

The BCFW recursion relations for $n$-point (S)YM amplitudes are formulated with the use of the on-shell amplitudes depending on deformed momentum spinors of, say, 1-st and n-th of the scattered particles. The BCFW deformation rule reads

$$
\begin{aligned}
\lambda_{(n)}^{A} \mapsto \widehat{\lambda_{(n)}^{A}}=\lambda_{(n)}^{A}+z \lambda_{(1)}^{A}, & \bar{\lambda}_{(n)}^{\dot{A}} \mapsto \widehat{\bar{\lambda}_{(n)}^{\dot{A}}}=\bar{\lambda}_{(n)}^{\dot{A}}, \\
\lambda_{(1)}^{A} \mapsto \widehat{\lambda_{(1)}^{A}}=\lambda_{(1)}^{A}, & \bar{\lambda}_{(1)}^{\dot{A}} \mapsto \widehat{\overline{\bar{\lambda}}_{(1)}^{\dot{A}}}=\bar{\lambda}_{(1)}^{\dot{A}}-z \bar{\lambda}_{(n)}^{\dot{A}},
\end{aligned}
$$


where $z$ is a complex number. Notice that $\widehat{\bar{\lambda}_{(1, n)}^{\dot{A}}}$ differs from complex conjugate of $\widehat{\lambda_{(1, n)}^{A}}$, so that the deformed momenta of 1 -st and n-th particle

$$
p_{(n)}^{a} \mapsto \widehat{p_{(n)}^{a}}(z)=p_{(n)}^{a}+z q^{a}, \quad p_{(1)}^{a} \mapsto \widehat{p_{(1)}^{a}}(z)=p_{(1)}^{a}-z \bar{q}^{a}
$$

are complex. It is important that they remain light-like

$$
\widehat{p_{(n)}^{a}} \widehat{p_{(n) a}}=0, \quad \widehat{p_{(1)}^{a}} \widehat{p_{(1) a}}=0
$$

and hence can be used as arguments of an on-shell amplitude. In (2.14) the vector $q^{a}$ is constructed as bilinear of the 1-st left and n-th right bosonic spinors,

$$
q^{A \dot{A}}=q^{a} \tilde{\sigma}_{a}^{A \dot{A}}=\lambda_{(1)}^{A} \bar{\lambda}_{(n)}^{\dot{A}} .
$$

As a result, it is complex, light-like and orthogonal both to 1-st and n-th momentum,

$$
q^{a} q_{a}=0, \quad p_{(n)}^{a} q_{a}=0, \quad p_{(1)}^{a} q_{a}=0 .
$$

One can easily check the lightlikeness of complex deformed momenta (2.15) using just (2.17) and (2.14).

The BCFW recurrent relations for tree amplitudes of $\mathrm{D}=4$ gluons read $[16,19]$

$$
\begin{aligned}
& \mathcal{A}^{(n)}\left(p_{1}, p_{2}, \ldots ; p_{n}\right)= \\
& =\sum_{h} \sum_{l=2}^{n} \mathcal{A}_{h}^{(l+1)}\left(\widehat{p}_{1}\left(z_{l}\right) ; p_{2} ; \ldots ; p_{l} ; \widehat{P_{\Sigma_{l}}}\left(z_{l}\right)\right) \frac{1}{\left(P_{\Sigma_{l}}\right)^{2}} \mathcal{A}_{-h}^{(n-l+1)}\left(-\widehat{P_{\Sigma_{l}}}\left(z_{l}\right), p_{l+1} ; \ldots ; \widehat{p_{n}}\left(z_{l}\right)\right)
\end{aligned}
$$

where $h$ is the helicity of intermediate state with momentum $\widehat{P_{\Sigma_{l}}}\left(z_{l}\right)$. Notice that, for shortness, we included the momentum conservation delta functions inside the amplitudes (cf. (2.11)). In the denominator of (2.18) we find the (minus) sum of the first $l$ undeformed momenta,

$$
P_{\Sigma_{l}}^{a}=-\sum_{m=1}^{l} p_{m}^{a},
$$

while the arguments contain the sum of corresponding deformed momenta

$$
\widehat{P_{\Sigma_{l}}^{a}}(z)=-\sum_{m=1}^{l} \widehat{p_{m}^{a}}(z)
$$

with the specific $l$-dependent value of the complex parameter $z$,

$$
z_{l} \equiv z_{P_{\Sigma_{l}}}(q)=\frac{P_{\Sigma_{l}}^{a} P_{\Sigma_{l} a}}{2 P_{\Sigma_{l}}^{b} q_{b}} .
$$

This values is designed in such a way that the sum of deformed momenta becomes light-like $\left(\widehat{P_{\Sigma_{l}}^{a}}\left(z_{l}\right)\right)^{2}=0$, and, hence, the r.h.s. of (2.18) contains on-shell amplitudes, although some of the light-like momenta they depend on are complex. 
Schematically one can write (2.18) in the form [20]

$$
\mathcal{A}=\sum_{\hat{P}} \sum_{h} \widehat{\mathcal{A}}_{h}\left(z_{P}\right) \frac{1}{P^{2}} \widehat{\mathcal{A}}_{-h}\left(z_{P}\right),
$$

in which in denominator $P$ is a sum of a subset of momenta, as in (2.19) above, $z_{P}$ is related to $P$ by (2.21), and $\sum_{\hat{P}}$ represents symbolically the sum over the division of the set of particle momenta on two subsets (the sum over such divisions is implicit in the expression (2.18) above).

\section{$2.3 \mathcal{N}=4 \mathrm{SYM}$ and $\mathcal{N}=8$ SUGRA superamplitudes and generalized BCFW}

One can also collect the data of all the n-particle amplitudes of the fields of SYM supermultiplet by considering a superfield amplitude, called superamplitude, depending, besides the set of $n$ bosonic spinors $\lambda_{(i)}^{A}=\left(\bar{\lambda}_{(i)}^{\dot{A}}\right)^{*}$, also on the set of $n$ complex fermionic coordinates $\eta_{(i)}^{q}$ carrying the index $q=1, \ldots, 4$ of the fundamental representation of $\mathrm{SU}(4)$ :

$$
\mathcal{A}(1 ; \ldots ; n)=\mathcal{A}\left(\lambda_{(1)}, \bar{\lambda}_{(1)}, \eta_{(1)} ; \ldots ; \lambda_{(n)}, \bar{\lambda}_{(n)}, \eta_{(n)}\right) .
$$

The fact staying beyond the above superfield representation for SYM amplitudes is that the unconstrained superfield depending on the above bosonic spinor and Grassmann variable $\eta^{q}$, but not on its complex conjugate $\bar{\eta}_{q}=\left(\eta^{q}\right)^{*}$,

$$
\Phi\left(\lambda, \bar{\lambda}, \eta^{q}\right)=f^{(+)}+\eta^{q} \chi_{q}+\frac{1}{2} \eta^{q} \eta^{p} s_{p q}+\frac{1}{3 !} \eta^{q} \eta^{p} \eta^{r} \epsilon_{r p q s} \bar{\chi}^{s}+\frac{1}{4 !} \eta^{q} \eta^{p} \eta^{r} \eta^{s} \epsilon_{r p q s} f^{(-)}
$$

describes the on-shell degrees of freedom of the $\mathcal{N}=4 \mathrm{SYM}$ multiplet provided it has a super-helicity +1 , i.e. if it obeys

$$
\hat{h} \Phi\left(\lambda, \bar{\lambda}, \eta^{q}\right)=\Phi\left(\lambda, \bar{\lambda}, \eta^{q}\right), \quad q=1, \ldots, 4,
$$

with

$$
2 \hat{h}:=\lambda^{A} \frac{\partial}{\partial \lambda^{A}}-\bar{\lambda}^{\dot{A}} \frac{\partial}{\partial \bar{\lambda}^{\dot{A}}}+\eta^{q} \frac{\partial}{\partial \eta^{q}} .
$$

In this sense one calls the superfield (2.24) and the superspace with coordinates $\left(\lambda, \bar{\lambda}, \eta^{q}\right)$ it is defined on the on-shell superfield and on-shell superspace, respectively. The component fields in (2.24) describe photons (or gluons) of helicity $+1\left(f^{(+)}=f^{(+)}(\lambda, \bar{\lambda})\right)$ and $-1\left(f^{(-)}=f^{(-)}(\lambda, \bar{\lambda})\right)$, photinos (or gluinos) of helicity $+1 / 2\left(\chi_{q}=\chi_{q}(\lambda, \bar{\lambda})\right)$ and $-1 / 2$ $\left(\bar{\chi}^{q}=\bar{\chi}^{q}(\lambda, \bar{\lambda})\right)$, and 6 scalars of the maximal $\mathcal{N}=4 \mathrm{SYM}$ multiplet $\left(s_{p q}=s_{[p q]}(\lambda, \bar{\lambda})\right)$.

The superfield amplitudes (superamplitudes) (2.23) of $\mathcal{N}=4$ SYM theory, which can be considered as multiparticle counterparts of the superfields $\Phi\left(\lambda, \bar{\lambda}, \eta^{q}\right)$, obey $n$ superhelicity constraints (with the same eigenvalue +1 )

$$
\begin{aligned}
\hat{h}_{(i)} \mathcal{A}\left(\left\{\lambda_{(i)}, \bar{\lambda}_{(i)}, \eta_{(i)}^{q}\right\}\right) & =\mathcal{A}\left(\left\{\lambda_{(i)}, \bar{\lambda}_{(i)}, \eta_{(i)}^{q}\right\}\right), \quad q=1, \ldots, 4, \quad i=1, \ldots, n, \\
2 \hat{h}_{(i)}: & =\lambda_{(i)}^{A} \frac{\partial}{\partial \lambda_{(i)}^{A}}-\bar{\lambda}_{(i)}^{\dot{A}} \frac{\partial}{\partial \bar{\lambda}_{(i)}^{\dot{A}}}+\eta_{i}^{q} \frac{\partial}{\partial \eta_{i}^{q}} .
\end{aligned}
$$


Similarly, the $\mathcal{N}=8$ supergravity multiplet is described by chiral superfield on an $\mathcal{N}=8$ on-shell superspace with 2 complex bosonic and 8 complex fermionic coordinates $\left(\lambda^{A}, \bar{\lambda}^{\dot{A}}, \eta^{q}\right), q=1, \ldots, 8$, which has superhelicity +2 , i.e. obeys

$$
\hat{h} \Phi\left(\lambda, \bar{\lambda}, \eta^{q}\right)=2 \Phi\left(\lambda, \bar{\lambda}, \eta^{q}\right), \quad q=1, \ldots, 8
$$

with $\hat{h}$ defined by (2.26) with $q=1, \ldots, 8$. The $n$-particle tree superamplitude of $\mathcal{N}=8$ supergravity can be described as a function in a direct product of $n$ copies of such an $\mathcal{N}=8$ on-shell superspace, depending holomorphically on the fermionic coordinates and carrying superhelicity +2 on each of the set of 2 complex bosonic and 8 complex fermionic coordinates $\left(\lambda_{(i)}^{A}, \bar{\lambda}_{(i)}^{\dot{A}}, \eta_{(i)}^{q}\right)$ corresponding to external legs,

$$
\hat{h}_{(i)} \mathcal{A}\left(\left\{\lambda_{(i)}, \bar{\lambda}_{(i)}, \eta_{(i)}^{q}\right\}\right)=2 \mathcal{A}\left(\left\{\lambda_{(i)}, \bar{\lambda}_{(i)}, \eta_{(i)}^{q}\right\}\right), \quad q=1, \ldots, 8
$$

To write the superfield generalization of the BCFW relations (2.18) for tree superamplitudes of $\mathcal{N}=8$ supergravity and $\mathcal{N}=4$ SYM [19, 20], one should supplements the deformations of the bosonic spinors (2.12), (2.13) by the deformation of the fermionic variables $\eta_{i}^{q}=\left(\bar{\eta}_{q i}\right)^{*}$ corresponding to 1 -st and $n$-th particles,

$$
\eta_{(n)}^{q} \mapsto \widehat{\eta_{(n)}^{q}}(z)=\eta_{(n)}^{q}+z \eta_{(1)}^{q}, \quad \eta_{(1)}^{q} \mapsto \widehat{\eta_{(1)}^{q}}(z)=\eta_{(1)}^{q}
$$

Notice that, actually, the fermionic variable of the 1-st particle has not been changed [19,20].

The schematic form of the generalization of the BCFW recurrent relations for $\mathcal{N}=4$ SYM superamplitudes reads

$$
\mathcal{A}=\sum_{\hat{P}} \int d^{4} \eta_{\hat{P}} \mathcal{A}_{L}\left(z_{\hat{P}}\right) \frac{1}{P^{2}} \mathcal{A}_{R}\left(z_{\hat{P}}\right)
$$

A more explicit form can be easily restored by comparison with (2.22) and (2.18), also taking into account the deformation of not only bosonic spinors (2.12), (2.13) but also fermionic variables, (2.31). A new element is the Grassmann integration over the fermionic variable corresponding to the intermediate states, $\eta_{\hat{P}}$.

The BCFW recurrent relations allow to construct all higher points on-shell superamplitudes from the basic 3-point superamplitudes. One of the aim of our work, the first stages of which are the subject of the present paper, was to find the generalization of these relations for the case of superamplitudes of 11D supergravity and 10D SYM. In sections 6-8 we will present and study a candidate for such a generalization (see [30] for brief description of $\mathrm{D}=11$ case). As we show, these however suffers an unwanted dependence on the deformation vector which is not fixed uniquely in higher dimensional cases. Thus the bottom line of the results of present paper will be the properties of the constrained on-shell superamplitudes of 10D SYM and 11D SUGRA, the counterparts of (2.23), and the equations they satisfy, the counterparts of (2.27) and (2.30). 


\subsection{Massless superparticle mechanics and on-shell superfields}

The on-shell superfields describing the states of maximal D=4 SYM and maximal supergravity can be obtained by quantization of the massless $\mathcal{N}=4$ and $\mathcal{N}=8$ superparticle mechanics defined by the following Ferber-Shirafuji action $[6,7]$

$$
S_{4 D}=\int d \tau \lambda_{A} \bar{\lambda}_{\dot{A}}\left(\partial_{\tau} X^{A \dot{A}}-i \partial_{\tau} \Theta_{q}^{A} \bar{\Theta}^{\dot{A} q}+i \Theta_{q}^{A} \partial_{\tau} \bar{\Theta}^{\dot{A} q}\right), \quad q=1, \ldots, \mathcal{N} .
$$

We review this well known procedure here as a guide in our search for the on-shell superfield description of 10D SYM and 11D supergravity multiplets which then will be generalized to $10 \mathrm{D}$ and $11 \mathrm{D}$ tree superamplitudes.

\subsubsection{Twistor form of the massless superparticle action}

Just by moving the derivative with the use of Leibnitz rules, we can write the action (2.33) in the form

$$
\begin{aligned}
S_{4 D} & =\int d \tau\left(\bar{\lambda}_{\dot{A}} \partial_{\tau} \mu^{\dot{A}}-\mu^{A} \partial_{\tau} \lambda_{A}-2 i \partial_{\tau} \eta_{q} \bar{\eta}^{q}\right)= \\
& =\int d \tau\left(\partial_{\tau} \lambda^{A} \mu_{A}-\partial_{\tau} \mu_{\dot{A}} \bar{\lambda}^{\dot{A}}-2 i \partial_{\tau} \eta_{q} \bar{\eta}^{q}\right), \quad q=1, \ldots, \mathcal{N},
\end{aligned}
$$

where

$$
\begin{aligned}
\mu^{\dot{A}} & =\lambda_{A} X_{L}^{A \dot{A}}, \quad X_{L}^{A \dot{A}}=X^{A \dot{A}}+i \Theta_{q}^{A} \bar{\Theta}^{\dot{A} q}=\left(X_{R}^{A \dot{A}}\right)^{*}, \\
\eta_{q} & =\Theta_{q}^{A} \lambda_{A}=\left(\bar{\eta}^{q}\right)^{*} .
\end{aligned}
$$

Eqs. (2.35) and (2.36) are called Penrose incidence relations. They define super-spacetime as a surface in the supertwistor superspace with complex coordinates $\left(\lambda_{A}, \mu^{\dot{A}}, \eta_{q}\right)[2,3,6]^{6}$ and describe the general solution of the 'helicity constraint'

$$
2 h=\bar{\mu}^{A} \lambda_{A}-\bar{\lambda}_{\dot{A}} \mu^{\dot{A}}+2 i \eta_{q} \bar{\eta}^{q} \approx 0 .
$$

\subsubsection{Hamiltonian mechanics in twistor formulation}

In the generic case the canonical momenta are defined as derivative of the Lagrangian with respect to velocities,

$$
\mathcal{P}_{M}=(-)^{M} \frac{\partial L}{\partial\left(\partial_{\tau} Y^{M}\right)},
$$

\footnotetext{
${ }^{6}$ To be more precise, (2.35) and (2.36) define the super-generalization of the space of light-like geodesics in super-spacetime as a surface in the space of supertwistors. This statement is tantamount to the observation that (2.35) and (2.36) are invariant under the transformations

$$
\delta X^{A \dot{A}}=t \lambda^{A} \bar{\lambda}^{\dot{A}}+i \lambda^{A} \kappa_{q} \bar{\Theta}^{\dot{A} q}-i \Theta_{q}^{A} \bar{\kappa}^{q} \bar{\lambda}^{\dot{A}}, \quad \delta \Theta_{q}^{A}=\kappa_{q} \lambda^{A}, \quad \delta \bar{\Theta}^{\dot{A} q}=\bar{\kappa}^{q} \bar{\lambda}^{\dot{A}}
$$

with an arbitrary real bosonic parameter $t$ and $\mathcal{N}$ arbitrary fermionic parameters $\kappa_{q}$. The fermionic transformations can be associated with the $\kappa$-symmetry of the massless superparticle action (2.33) which was observed a bit latter in [80] (see earlier [81] for the $\kappa$-symmetry of massive $\mathcal{N}=2$ superparticle).
} 
where

$$
(-)^{M}:=(-)^{\varepsilon\left(Y^{M}\right)}=\left\{\begin{array}{l}
+1 \text { for bosonic } Y^{M} \\
-1 \text { for fermionic } Y^{M} .
\end{array}\right.
$$

The canonical Poisson brackets are

$$
[\quad, \quad\}_{P . B .}=\frac{\partial}{\partial Y^{M}} \quad \frac{\partial}{\partial \mathcal{P}_{M}} \quad-(-)^{M} \frac{\partial}{\partial \mathcal{P}_{M}} \quad \frac{\partial}{\partial Y^{M}} \quad,
$$

so that $\left[\mathcal{P}_{M}, \mathcal{P}_{N}\right\}_{P . B .}=0=\left[Y^{M}, Y^{N}\right\}_{P . B .}$ and

$$
\left[Y^{N}, \mathcal{P}_{M}\right\}_{P . B .}=\delta_{M}^{N}=-(-)^{M N}\left[\mathcal{P}_{M}, Y^{N}\right\}_{P . B .} .
$$

Canonical Hamiltonian $\mathcal{H}$ is defined by Legandre transform of the Lagrangian $L$

$$
\mathcal{H}=(-)^{M} \partial_{\tau} Y^{M} \mathcal{P}_{M}-L,
$$

and the Hamiltonian equations of motion have the form $\partial_{\tau} Y^{M}=\left[Y^{M}, \mathcal{H}\right]_{P . B}$.

In our dynamical system described by $S_{4 D}=\int d \tau L_{4 D}$ of (2.34), the definition (2.38) results in the (second class) constraints which identify the bosonic spinor $\mu$ with the momentum of the bosonic spinor $\lambda$, and the momentum of the fermionic coordinate $\eta_{q}$ with its complex conjugate $\bar{\eta}^{q}$, so that the basic nonvanishing Poisson brackets (actually, these are Dirac brackets) read

$$
\begin{aligned}
{\left[\mu_{A}, \lambda^{B}\right]_{P . B .} } & =-\delta_{A}^{B}, \quad\left[\mu_{\dot{A}}, \bar{\lambda}^{\dot{B}}\right]_{P . B .}=-\delta_{\dot{A}}^{\dot{B}} \quad\left(=\left[\bar{\lambda}_{\dot{B}}, \mu^{\dot{A}}\right]_{P . B .}\right) \\
\left\{\eta_{q}, \bar{\eta}^{p}\right\}_{P . B .} & =-\frac{i}{2} \delta_{q}^{p} .
\end{aligned}
$$

The only constraint (after getting read of the second class constraints by passing to Dirac brackets $(2.42),(2.43)$ ) is $(2.37)$. It generates (on the Dirac brackets) U(1) gauge symmetry characteristic for the $D=4$ (super)twistor approach.

\subsubsection{Quantization and appearance of the on-shell superfields}

Quantization implies the replacement of canonical variables by operators and Poisson brackets by graded commutators (i.e. commutators or anti-commutators)

$$
[\ldots, \ldots\}_{P . B .} \mapsto \frac{1}{i \hbar}[\ldots, \ldots\} .
$$

In the coordinate representation

$$
\mu_{A} \mapsto-i \hbar \frac{\partial}{\partial \lambda^{A}}, \quad \mu_{\dot{A}} \mapsto-i \hbar \frac{\partial}{\partial \bar{\lambda}^{\dot{B}}}, \quad \bar{\eta}^{p} \mapsto \frac{\hbar}{2} \frac{\partial}{\partial \eta_{p}}
$$

(below we will set $\hbar=1$ ) and the 'wavefunction' (or 'classical field') depends holomorphically on the fermionic variable, i.e. it is given by an on-shell superfield (2.24). The constraints (2.37) should be imposed on the wave function in its operator form (2.26), 
$h \mapsto-i \hbar \hat{h}$. Actually, the operator ordering can produce an arbitrary constant contribution so that the quantum constraints for the wavefunction of massless superparticle in $\mathcal{N}$-extended $D=4$ on-shell superspace reads

$$
(\hat{h}-s) \Phi=0, \quad 2 s \in \mathbb{Z} .
$$

The fact that $s$ should be quantized in half-integer unit of $\hbar$ follows from the requirement that $\Phi$ is a single-valued function of complex variables $\lambda_{A}$, while the choice $s=1$ for $\mathcal{N}=4$ and $s=2$ for $\mathcal{N}=8$ is made from 'physical reasons' (of absence in the multiplet under consideration of gravity in the former and of higher spin fields in the latter case).

The equation (2.42) for the wavefunction of superparticle suggests that the scattering amplitudes obey eqs. (2.27) and (2.30) in the case of $\mathcal{N}=4 \operatorname{SYM}(s=1)$ and $\mathcal{N}=8$ supergravity $(s=2)$, respectively.

\subsubsection{From $\mathrm{D}=4$ to $\mathrm{D}=10$ and $\mathrm{D}=11$}

In this paper our main interest is in the properties of tree amplitudes and superamplitudes of the $\mathrm{D}=10 \mathrm{SYM}$ and $\mathrm{D}=11$ SUGRA theories. Our way to arrive at the appropriate spinor helicity and on-shell superfield formalism will pass through quantization of the $\mathrm{D}=10$ and $\mathrm{D}=11$ counterparts of the Ferber-Shirafuji action $(2.33)[33,37]$ which we will describe in the next section. Instead of unconstrained $4 \mathrm{D}$ Weyl spinors $\lambda_{A}, \bar{\lambda}_{\dot{A}}$ these $\mathrm{D}=10$ and $\mathrm{D}=11$ twistor-like actions use the spinor moving frame variables, also called Lorentz harmonics, parametrizing the celestial sphere $S^{(D-2)}$ as a coset of D-dimensional Lorentz group $\mathrm{SO}(1, D-1)$ over its Borel subgroup $[\mathrm{SO}(1,1) \otimes \mathrm{SO}(D-2)] \otimes K_{D-2}[31,32,35]$.

The counterparts of these $\frac{\mathrm{SO}(1, D-1)}{[\mathrm{SO}(1,1) \otimes \mathrm{SO}(D-2)] \otimes K_{D-2}}$ Lorentz harmonics, spinor frame variables associated to each of the scattered particles, will be used below to construct spinor helicity formalism for amplitudes of $D=10 \mathrm{SYM}$ and $D=11$ SUGRA.

In the case of $D=10$ and $D=11$ superparticle, the spinor moving frame variables can be considered as additional coordinate of target superspace which then can be called Lorentz harmonic superspace. ${ }^{7}$ Their presence allows to perform a change of variables similar to passing to the twistor variables in (2.34), which can be seen now as change of coordinates of this from 'central' basis, given by the standard bosonic vector and fermionic spinor coordinates of $\mathrm{D}=10$ and $\mathrm{D}=11$ superspaces plus the above mentioned spinor harmonics, to a different, so-called analytical coordinate basis. ${ }^{8}$ The quantization of superparticle in this analytical basis will provide us with the on-shell superfields describing the degrees of freedom of 10D SYM and 11D SUGRA. The tree superamplitudes will be constructed as multiparticle generalizations of these on-shell superfields.

\footnotetext{
${ }^{7}$ See [82-84] for the concept of harmonic superspace.

${ }^{8}$ The quantization of superparticle in the analytical basis of Lorentz harmonic superspace was discussed for the first time in $[78,79]$ using the vector moving frame variables (vector harmonics) only. The formulation of $\mathrm{D}=4$ Ferber-Shirafuji superparticle in terms of corresponding spinor moving frame variables (spinor harmonics) can be found in [85]. There the massless superparticle was considered both in the central and in the analytic coordinate basis of $\mathrm{D}=4$ Lorentz harmonic superspace.
} 


\section{Superparticle in ten and eleven dimensions. Spinor moving frame formulation}

\subsection{Brink-Schwarz action for massless superparticle in $\mathrm{D}=10$ and $\mathrm{D}=11$}

The Brink-Schwarz superparticle action can be defined in any dimensions

$$
S_{B S}[X, p, \theta]=\int d \tau\left(p_{a}\left(\partial_{\tau} X^{a}-i \partial_{\tau} \theta \Gamma^{a} \theta\right)-\frac{e}{2} p^{a} p_{a}\right) .
$$

Here $X^{a}=X^{a}(\tau)$ and $\theta^{\alpha}=\theta^{\alpha}(\tau)$ are bosonic and fermionic coordinate functions, $p_{a}=$ $p_{a}(\tau)$ is auxiliary momentum variable, $e(\tau)$ is an einbein field playing the role of Lagrange multiplier, and $\partial_{\tau} \theta \Gamma^{a} \theta=\partial_{\tau} \theta^{\alpha} \Gamma_{\alpha \beta}^{a} \theta^{\beta}$.

In $\mathrm{D}=11$ case $a=0,1, \ldots, 9,10, \alpha=1, \ldots, 32$, the real fermionic $\theta^{\alpha}$ are transformed as Majorana spinor of $\mathrm{SO}(1,10)$ and $\Gamma_{\alpha \beta}^{a}=\Gamma_{\beta \alpha}^{a}=\Gamma_{\alpha}^{a \gamma} C_{\gamma \beta}$ are the products of 11D Dirac matrices $\Gamma_{\alpha}^{a \gamma}=-\left(\Gamma_{\alpha}^{a \gamma}\right)^{*}$ obeying the Clifford algebra, $\Gamma^{a} \Gamma^{b}+\Gamma^{b} \Gamma^{a}=$ $2 \eta^{a b} \mathbb{I}_{32 \times 32}$, and of $11 \mathrm{D}$ charge conjugation matrix $C_{\gamma \beta}=-C_{\beta \gamma}=-\left(C_{\gamma \beta}\right)^{*}$. We will also use $\tilde{\Gamma}^{a \alpha \beta}=\tilde{\Gamma}^{a \alpha \beta}=C^{\alpha \gamma} \Gamma_{\gamma}^{a \beta}$.

In $\mathrm{D}=10$ case the fermionic $\theta^{\alpha}$ is real Majorana-Weyl (MW) spinor of $\mathrm{SO}(1,9)$, $\alpha=1, \ldots, 16$, and $\Gamma_{\alpha \beta}^{a}=\sigma_{\alpha \beta}^{a}=\sigma_{\beta \alpha}^{a}$ obey $\sigma^{a} \tilde{\sigma}^{b}+\sigma^{b} \tilde{\sigma}^{a}=2 \eta^{a b} \mathbb{I}_{16 \times 16}$ in product with their upper-indices counterpart $\tilde{\Gamma}^{a \alpha \beta}=\tilde{\sigma}^{a \alpha \beta}=\tilde{\sigma}^{a \beta \alpha}$. There is no covariant way of rising or lowering Mayorana-Weyl spinor indices in $\mathrm{D}=10$ as these correspond to different chiralities (in other words, the charge conjugation matrix does not exist in $\mathrm{D}=10 \mathrm{MW}$ spinor representation).

In any dimension D the action (3.1) is invariant under rigid supersymmetry

$$
\delta_{\varepsilon} X^{a}=i \theta \Gamma^{a} \varepsilon, \quad \delta_{\varepsilon} \theta^{\alpha}=\varepsilon^{\alpha}, \quad \delta_{\varepsilon} p_{a}=0, \quad \delta_{\varepsilon} e=0,
$$

and under local fermionic kappa-symmetry [80] (see also earlier [81])

$$
\delta_{\kappa} X^{a}=-i \theta \Gamma^{a} \delta_{\kappa} \theta, \quad \delta_{\kappa} \theta^{\alpha}=p_{a} \tilde{\Gamma}^{a \alpha \beta} \kappa_{\beta}, \quad \delta_{\kappa} p_{a}=0, \quad \delta_{\kappa} e=-4 i \partial_{\tau} \theta \kappa .
$$

\subsection{Moving frame formulation of massless superparticle mechanics}

In this section we follow closely the discussion in [37]. The variation of (3.1) with respect to the Lagrange multiplier $e(\tau)$ results in the mass-shell condition

$$
p_{a} p^{a}=0
$$

This is algebraic equation and thus, knowing its solution we can substitute it back to the action. Hence, formally we can write the action (3.1) in the equivalent form

$$
S_{B S}^{\prime}=\left.\int d \tau\left(\partial_{\tau} X^{a}-i \partial_{\tau} \theta \Gamma^{a} \theta\right) p_{a}\right|_{p^{2}=0} .
$$

A particular solution of the constraint (3.4) is given by

$$
p_{a}=\rho(1,0, \ldots, 0,-1)
$$


with arbitrary $\rho(\tau)$ describing the energy of the massless particle. Any other solution can be obtained from this by performing a $\tau$-dependent $\mathrm{SO}(1, D-1)$ Lorentz transformation

$$
u_{a}^{(b)}(\tau) \in \operatorname{SO}(1, D-1) \quad \Leftrightarrow \quad u_{a}^{(b)} u^{a(c)}=\eta^{(b)(c)}=\operatorname{diag}(+1,-1, \ldots,-1) .
$$

Hence, the general solution of the mass-shell constraint reads

$$
p_{a}(\tau)=u_{a}^{(b)} p_{(b)}=\rho(\tau)\left(u_{a}^{0}-u_{a}^{(D-1)}\right)=: \rho^{\#}(\tau) u_{a}^{=}(\tau),
$$

where we have supplied the energy variable $\rho$ of (3.6) by the indices ${ }^{\#}={ }^{++}$which indicate the invariance of the solution (3.8) under arbitrary scaling of $u_{a}^{=}$compensated by an opposite scaling of $\rho^{\#}$. As we will see below this scaling symmetry may be associated with $\mathrm{SO}(1,1)$ subgroup of Lorentz group $\mathrm{SO}(1, D-1)$.

By construction, the vector $u_{a}^{=}(\tau)=\left(u_{a}^{0}-u_{a}^{(D-1)}\right)$ is light-like. Substituting this general solution into the Brink-Schwarz action we arrive at

$$
S=\int d \tau \rho^{\#} u_{a}^{=}\left(\partial_{\tau} X^{a}-i \partial_{\tau} \theta \Gamma^{a} \theta\right), \quad u_{a}^{=} u^{a=}=0 .
$$

This action describes moving frame formulation of massless superparticle, as it includes a light-like vector (3.8) which we have introduced as a difference of two columns of the Lorentz group valued matrix (3.7). It is convenient to consider this matrix to be an element of proper Lorentz group simply connected to unity $S O^{\uparrow}(1, D-1)$ (see $[31,32]$ ), and to write this matrix explicitly in terms of light-like vector $u_{a}^{=}$and its complementary $u_{a}^{\#}(\tau)=\left(u_{a}^{0}+u_{a}^{(D-1)}\right)$,

$$
u_{a}^{(b)}(\tau)=\left(\frac{1}{2}\left(u_{a}^{=}+u_{a}^{\#}\right), u_{a}^{i}, \frac{1}{2}\left(u_{a}^{\#}-u_{a}^{=}\right)\right) \in S O^{\uparrow}(1, D-1) .
$$

At this stage it might seem that we just have substituted a simple action (3.5) by an almost identical but a bit more complicated (3.9) without any benefit. The benefit however is present (although still hidden) as the clear geometrical and group theoretical meaning of moving frame will allow us to extract a square root of (3.10), which gives us the spinor moving frame providing the $10 \mathrm{D}$ and $11 \mathrm{D}$ counterparts of spinor helicity variables. We will see that, when the moving frame variables are understood as constructed from the spinor moving fame variables, the action (3.9) becomes a D-dimensional counterpart of the $\mathrm{D}=4$ Ferber-Shirafuji action (2.33).

But before introducing the spinor moving frame, let us discuss some technical details about moving frame variables.

\subsection{Properties of moving frame variables (vector harmonics)}

The statement (3.10) is equivalent to preservation of the flat Lorentzian metric under similarity transformations with the moving frame matrices $U=\left\|u_{a}^{(b)}\right\|$, i.e. to $U^{T} \eta U=$ $\eta$ and $U \eta U^{T}=\eta$. These equations can be equivalently written as $u_{a}^{(b)} u^{a(c)}=\eta^{(b)(c)}$ (see (3.7)), which splits into

$$
\begin{array}{rlrl}
u_{a}^{\bar{a}} u^{a=} & =0, & \\
u_{a}^{\#} u^{a \#} & =0, & & u_{a}^{=} u^{a \#}=2, \\
u_{a}^{I} u^{a=}=0 & =u_{a}^{I} u^{a \#}, & u_{a}^{I} u^{a J}=-\delta^{I J},
\end{array}
$$


and as

$$
\delta_{a}{ }^{b}=\frac{1}{2} u_{a}^{=} u^{b \#}+\frac{1}{2} u_{a}^{\#} u^{b=}-u_{a}^{I} u^{b I}
$$

respectively.

The set of constrained variables (3.10) considered as worldline fields, $u_{a}^{\overline{=}}=u_{a}^{\overline{\bar{a}}}(\tau)$, etc., describe a moving frame attached to every point of the worldsheet in such a way that canonical momentum of $X^{a}(\tau)$ field is proportional to $u_{a}^{=}(\tau)$ (as it can be seen from (3.8)). Hence these fields can be called moving frame fields or moving frame variables.

On the other side, one can treat the model (3.9) as a model in superspace enlarged by additional bosonic directions parametrizing $\mathrm{SO}(1, D-1)$ group or its coset. Such an enlarged superspace, first introduced in [78] is called Lorentz harmonic superspace. ${ }^{9} u_{a}^{=}, u_{a}^{\#}$ and $u_{a}^{I}$ constrained by (3.11)-(3.14) (or equivalently by the condition (3.10) on $u_{a}^{(b)}$ matrix collecting them) can be considered as coordinates of the 'internal' sector of this superspace and can be called Lorentz harmonic variables. Of course these name can be applied as well to the worldline fields, $u_{a}^{(b)}(\tau)$, etc., so that the names Lorentz harmonics and moving frame variables can be used as synonyms.

Notice also that the splitting (3.10) of the $\mathrm{SO}(1, \mathrm{D}-1)$ valued matrix $u_{a}^{(b)}(\tau)$ is invariant under the subgroup $\mathrm{SO}(1,1) \times \mathrm{SO}(D-2)$ of the Lorentz group $\mathrm{SO}(1, D-1)$ so that in a model which is gauge invariant under local $\mathrm{SO}(1,1) \times \mathrm{SO}(D-2)$ transformations, the fields $u_{a}^{(b)}=\left(u_{a}^{=}, u_{a}^{\#}, u_{a}^{I}\right)$ can be identified as homogeneous coordinates of the coset $\frac{\mathrm{SO}(1, D-1)}{\mathrm{SO}(1,1) \times \mathrm{SO}(D-2)}$,

$$
\left\{u_{a}^{=}, u_{a}^{\#}, u_{a}^{I}\right\}=\frac{\mathrm{SO}(1, D-1)}{\mathrm{SO}(1,1) \times \mathrm{SO}(D-2)} .
$$

The (spinor) moving frame variables used to describe (super)string model in its "twistorlike Lorentz harmonic formulation" of [39-41] parametrize this non-compact coset.

The superparticle model is more economic: of all $u_{a}^{(b)}(\tau) \in \mathrm{SO}(1, D-1)$ it contains only one light-like vector $u_{a}^{=}(\tau)$. Hence, if we treat this light-like vector as a part of moving frame matrix $u_{a}^{(b)}(\tau)$, this model is gauge invariant, besides $\mathrm{SO}(1,1) \times \mathrm{SO}(D-2)$, under such $\mathrm{SO}(1, D-1)$ transformations which mixes the complementary light-like vector $u_{a}^{\#}(\tau)$ with spacelike vectors $u_{a}^{I}(\tau)$. These transformation form an Abelian subgroup $K_{D-2}$ of the Lorentz group $\mathrm{SO}(1, D-1)$. The complete set of the gauge symmetries in the (spinor) moving frame sector of our superparticle model is thus a semidirect product $[\mathrm{SO}(1,1) \times$ $\mathrm{SO}(D-2)] \otimes K_{D-2}$ which is the so-called Borel subgroup of $\mathrm{SO}(1, D-1)$. The latter fact implies that the coset $\frac{\mathrm{SO}(1, D-1)}{[\mathrm{SO}(1,1) \times \mathrm{SO}(D-2)] \otimes K_{D-2}}$ is compact; it is isomorphic to the sphere $\mathbb{S}^{D-2}$ which (when the massless (super)particle model is considered) can be identified as celestial sphere of a D-dimensional observer [31, 32]. Resuming,

$$
\left\{u_{a}^{=}, u_{a}^{\#}, u_{a}^{I}\right\}=\frac{\mathrm{SO}(1, D-1)}{[\mathrm{SO}(1,1) \times \mathrm{SO}(D-2)] \otimes K_{D-2}}=\mathbb{S}^{D-2},
$$

\footnotetext{
${ }^{9}$ In $[78,79]$ the name 'light-cone superspace' was used. The term 'harmonic superspace' was introduced in the seminal papers [82, 83] where the off-shell description of $\mathcal{N}=2,3 \mathrm{SYM}$ theory and $\mathcal{N}=2$ supergravity in terms of unconstrained superfields have been constructed; see also [84].
} 
or, more schematically,

$$
\left\{u_{a}^{=}\right\}=\frac{\mathrm{SO}(1, D-1)}{[\mathrm{SO}(1,1) \times \mathrm{SO}(D-2)] \otimes K_{D-2}}=\mathbb{S}^{D-2} .
$$

Again, the above observations result in nontrivial consequences only after 'extracting the square roots' of the moving frame vectors, i.e. after reformulating this in terms of spinor moving frame variables, which are of interest for us as basic elements of D-dimensional spinor helicity formalism. Let us describe these in a notation especially suitable for $\mathrm{D}=10$ and $\mathrm{D}=11$ dimensional cases.

\subsection{Spinor moving frame variables (spinor harmonics)}

The basic fact allowing for extracting a square root of the moving frame matrix is the Lorentz invariance of D-dimensional Dirac (or Pauli) matrices $\Gamma^{a}$ and also of D-dimensional charge conjugation matrix $C_{\alpha \beta}$ if such exists in minimal D-dimensional spinor representation. The conditions of these Lorentz invariance can be written, in particular, for the moving fame matrix (3.10), (3.7),

$$
\begin{aligned}
& V \Gamma_{b} V^{T}=u_{b}^{(a)} \Gamma_{(a)}, \quad V^{T} \tilde{\Gamma}^{(a)} V=\tilde{\Gamma}^{b} u_{b}^{(a)}, \\
& V C V^{T}=C, \quad \text { if } \quad C \text { exists for given } D .
\end{aligned}
$$

These equations define the spinor moving frame matrix $V$ which takes values in the fundamental representation of the doubly covering group $\operatorname{Spin}(1, D-1)$ of the Lorentz group $\mathrm{SO}(1, D-1), V \in \operatorname{Spin}(1, D-1)$. It is $n \times n$ matrix, where $n$ is dimension of a minimal spinor representation of $D$-dimensional Lorentz group: $n=16$ for $\mathrm{D}=10$ and $n=32$ for $\mathrm{D}=11$.

The $\mathrm{SO}(1,1) \times \mathrm{SO}(D-2)$ invariant splitting $(3.7), u_{b}^{(a)}=\left(u_{b}^{\bar{y}}, u_{b}^{\#}, u_{b}^{I}\right)$, is reflected by splitting the spinor moving frame matrix on two rectangular blocks,

$$
V_{\alpha}^{(\beta)}=\left(v_{\alpha \dot{q}}^{+}, v_{\alpha q}^{-}\right) \in \operatorname{Spin}(1, D-1),
$$

The columns of two blocks of these matrices are enumerated by indices of (the same or different) representations of $\mathrm{SO}(D-2)$ subgroup of $\mathrm{SO}(1, D-1)$. In particular, in the case of $\mathrm{D}=10$, where the minimal MW spinor representation is 16 -dimensional, these are c- and s-spinor indices of $\mathrm{SO}(8)$,

$$
D=10: \quad \alpha=1, \ldots, 16, \quad \dot{q}=1, \ldots, 8, \quad q=1, \ldots, 8,
$$

while in $D=11$ these are two copies of the same real (Majorana) spinor representation of the $\mathrm{SO}(9)$ group,

$$
D=11: \quad \alpha=1, \ldots, 32, \quad q=\dot{q}=1, \ldots, 16, \quad v_{\alpha \dot{q}}^{+} \equiv v_{\alpha q}^{+} .
$$

The sign indices ${ }^{ \pm}$of two blocks, $v_{\alpha \dot{q}}^{+}$and $v_{\alpha q}^{-}$, of the spinor moving frame matrix (3.20) indicate their scaling properties with respect to the $\mathrm{SO}(1,1)$ transformations. 
Generically, working with spinor moving frame variables one cannot avoid the use of the inverse of the spinor moving frame matrix

$$
V_{(\beta)}^{\alpha}=\left(\begin{array}{c}
v_{q}^{+\alpha} \\
v_{\dot{q}}^{-\alpha}
\end{array}\right) \in \operatorname{Spin}(1, D-1)
$$

the blocks of which obey $V_{\alpha}^{(\beta)} V_{(\beta)}^{\gamma}:=v_{\alpha}^{-\dot{q}} v_{\dot{q}}^{+\gamma}+v_{q}^{-\alpha} v_{q}^{-\gamma}=\delta_{\alpha}^{\gamma}$ and

$$
\begin{array}{ll}
v_{q}^{+\alpha} v_{\alpha p}^{-}=\delta_{q p}, & v_{q}^{+\alpha} v_{\alpha \dot{p}}^{+}=0, \\
v_{\dot{q}}^{-\alpha} v_{\alpha q}^{-}=0, & v_{\dot{q}}^{-\alpha} v_{\alpha p}^{+}=\delta_{\dot{q} \dot{p} .} .
\end{array}
$$

In $\mathrm{D}=11$ the elements of the inverse spinor moving frame matrix can be constructed from the elements of (3.20) with the use of charge conjugation matrix

$$
D=11: \quad v_{q}^{ \pm \alpha}= \pm i C^{\alpha \beta} v_{\beta q}^{ \pm} .
$$

In $\mathrm{D}=10$ the charge conjugation matrix does not exist and we define the elements of (3.23) by the constraints (3.24).

For our dynamical system an especially important relations between the vector and spinor moving frame variables (vector and spinor Lorentz harmonics) (3.18) are

$$
\begin{aligned}
u_{a}^{=} \Gamma_{\alpha \beta}^{a} & =2 v_{\alpha q}{ }^{-} v_{\beta q}{ }^{-}, & & v_{q}^{-} \tilde{\Gamma}_{a} v_{p}^{-}=u_{a}^{=} \delta_{q p}, \\
u_{a}^{=} \tilde{\Gamma}^{a \alpha \beta} & =2 v_{\dot{q}}^{-\alpha} v_{\dot{q}}^{-\beta}, & & v_{\dot{q}}^{-} \Gamma_{a} v_{\dot{p}}^{-}=u_{a}^{=} \delta_{\dot{q} \dot{p} .} .
\end{aligned}
$$

The equations in the first line of (3.26) allow to state that $v_{\alpha q}^{-}$is the square root of the light-like moving frame vector $u_{a}^{=}$in the same sense as in $\mathrm{D}=4$ the bosonic spinor $\lambda_{A}$ can be called square root of the light-like 4-momentum due to eq. (2.1). The equations in the second line state the same for the block $v_{\dot{q}}^{-\alpha}$ of the inverse spinor moving frame matrix (3.23). This fact is nontrivial for $\mathrm{D}=10$, while for $\mathrm{D}=11 v_{\dot{q}}^{-\alpha} \equiv v_{q}^{-\alpha}$ is expressed through $v_{\alpha q}^{-}$by (3.25) and the equations in the second line of (3.26) follow from the first line.

Similar relations with other moving frame vectors involve the complementary harmonic variables $v_{\alpha q}^{+}$and $v_{\dot{q}}^{+\alpha}$ :

$$
\begin{aligned}
v_{\dot{q}}^{+} \tilde{\Gamma}_{a} v_{\dot{p}}^{+} & =u_{a}^{\#} \delta_{\dot{q} \dot{p}}, & 2 v_{\alpha \dot{q}}{ }^{+} v_{\beta \dot{q}}{ }^{+} & =\Gamma_{\alpha \beta}^{a} u_{a}^{\#}, \\
v_{q}^{+} \Gamma_{a} v_{p}^{+} & =u_{a}^{\#} \delta_{q p}, & 2 v_{q}^{+\alpha} v_{q}^{+\beta} & =\tilde{\Gamma}^{a \alpha \beta} u_{a}^{\#}, \\
v_{q}^{-} \tilde{\Gamma}_{a} v_{\dot{p}}^{+} & =u_{a}^{I} \gamma_{q \dot{p}}^{I}, & 2 v_{(\alpha \mid q}{ }^{-} \gamma_{q \dot{q}}^{I} v_{\mid \beta) \dot{q}}{ }^{+} & =\Gamma_{\alpha \beta}^{a} u_{a}^{I}, \\
v_{\dot{q}}^{-} \Gamma_{a} v_{p}^{+} & =-u_{a}^{I} \gamma_{p \dot{p}}^{I}, & 2 v_{\dot{q}}^{-(\alpha} \gamma_{q \dot{q}}^{I} v_{q}^{+\beta)} & =-\tilde{\Gamma}^{a \alpha \beta} u_{a}^{I} .
\end{aligned}
$$

Here, for $\mathrm{D}=10 \gamma_{p \dot{q}}^{I}=: \tilde{\gamma}_{\dot{q} p}^{I}$ are Klebsh-Gordan coefficients of $\mathrm{SO}(8)$ group, $q, p=1, \ldots, 8$ are s-spinor (8s) indices, $\dot{q}, \dot{p}=1, \ldots, 8$ are c-spinor ( $8 \mathrm{c}$ ) indices and $\mathrm{I}=1, \ldots, 8$ is $\mathrm{SO}(8)$ vector index (8v-index); all three representations are 8 dimensional in this case. For $\mathrm{D}=11 q, p \equiv$ $\dot{q}, \dot{p}=1, \ldots, 16$ are spinor indices of $\mathrm{SO}(9)$ and $\gamma_{q p}^{I}=\gamma_{p q}^{I}$ are $\mathrm{SO}(9)$ gamma matrices. For completeness, let us also repeat here that in $\mathrm{D}=10 \Gamma_{\alpha \beta}^{a}=\sigma_{\alpha \beta}^{a}, \tilde{\Gamma}_{a}^{\alpha \beta}=\tilde{\sigma}_{a}^{\alpha \beta}$ are generalized 10D Pauli matrices while for $\mathrm{D}=11 \tilde{\Gamma}_{a}^{\alpha \beta}=C^{\alpha \gamma} \Gamma_{a \gamma \delta} C^{\delta \beta}$. 


\subsection{Spinor moving frame formulation of the massless superparticle action}

Using (3.26), we can write (3.9) in the form

$$
S=\int d \tau \rho^{\#}\left(u_{a}^{=} \partial_{\tau} X^{a}-2 i \partial_{\tau} \theta^{\alpha} v_{\alpha q}^{-} \theta^{\beta} v_{\beta q}^{-}\right), \quad\left\{\begin{array}{l}
u_{a}^{=} u^{a=}=0, \\
u_{a}^{=} \Gamma_{\alpha \beta}^{a}=2 v_{\alpha q}^{-} v_{\beta q}^{-}, \\
v_{q}^{-} \tilde{\Gamma}_{a} v_{p}^{-}=u_{a}^{=} \delta_{q p} .
\end{array}\right.
$$

This counterpart of the $\mathrm{D}=4$ Ferber-Schirafuji action is the basis of the spinor moving frame formulation of massless D-dimensional superparticle (also called twistor-like Lorentz harmonic formulation) proposed and investigated in [33] for $\mathrm{D}=10$ and in [36-38] for 11D cases.

In distinction to the Brink-Schwarz formulation (3.1), the $\kappa$-symmetry of the spinor moving frame action (3.31) is irreducible,

$$
\delta_{\kappa} X^{a}=-i \theta \sigma^{a} v_{q}^{-} \kappa^{+q}, \quad \delta_{\kappa} \theta^{\alpha}=\kappa^{+q} v_{q}^{-\alpha}, \quad \delta_{\kappa} v_{q}^{-\alpha}=0=\delta_{\kappa} v_{\alpha \dot{q}}{ }^{-} .
$$

But moreover, as we will see below, in section 5.2, we can perform such a change of variables after which this local fermionic symmetry is gauge fixed automatically: the action in this basis contains only variables which are inert under $\kappa$-symmetry.

However, before passing to this issue, for which we will need to consider differential calculus on the space of spinor moving frame (Lorentz harmonic) variables, we would like to show that the $\mathrm{D}=10$ spinor helicity variables of Caron-Huot and O'Connell are actually spinor frame variables, and present the basic equations of this $\mathrm{D}=10$ spinor helicity formalism and of its $\mathrm{D}=11$ generalization.

\section{Spinorial frames, $\mathrm{D}=10$ spinor helicity formalism of Caron-Huot and O'Connell, and its $\mathrm{D}=11$ generalization}

In this section we show that spinor helicity formalism for $\mathrm{D}=10$ amplitudes proposed by Caron-Huot and O'Connell in [25] can be treated as spinor frame approach to the amplitudes, and use this observation to construct $\mathrm{D}=11$ generalization of this formalism.

It is worth stressing that in this paper, following the line of $[16,19,25]$ we will consider the on-shell amplitudes only and do not use neither (full field theory) Lagrangian nor Feynman diagrams in their derivation. Such pure on-shell approaches to the amplitudes (the set of which also incudes the unitary cut technique [9]) revived key ideas of the S-matrix program [86] aiming to restore the amplitudes mainly from the kinematics, symmetries and locality principle. For maximally supersymmetric $D=4$ theories such approaches have been quite successful resulting in a progress which cannot be reached with Feynman diagrams, see e.g. $[14,15]$ and refs therein. For higher dimensional cases, especially for $\mathrm{D}=10$ and $\mathrm{D}=11$, the on-shell (super)amplitude methods still need to be further elaborated and new ideas and new guides for the construction of the amplitudes are welcome. The aim of this paper is to contribute in such a development.

Particularly, in sections 5, 6 we use the statement that tree superamplitudes are multiparticle counterparts of the wavefunctions obtained in a suitable quantization of superparticle mechanics to find the small group index structure and transformation properties 
of the superamplitudes as well as the equations which obey these superamplitudes. In this section we discuss the tree amplitudes which appear as leading components of these superamplitudes and obtain the supersymmetric Ward identities relating several amplitudes. To introduce the amplitudes independently of superamplitudes, we consider them as multiparticle counterparts of the solution of the linearized equations of motion for the fields of 10D SYM and 11D SUGRA supermultiplets obtained with the use of spinor moving frame. This way also makes manifest how the supersymmetry transformations act on the amplitudes which, together with the evident fact that the amplitude for the process involving an odd number of fermions (counting all the incoming and outcoming particles) should vanish, gives us the above mentioned Ward identities.

Although we are not ready to reproduce the amplitudes just from the solution of the Ward identities (as suggests the S-matrix program), below (in section 8.2.) we will check that 3-point amplitudes with two fermionic legs suggested by the form of SYM (and superstring) vertices found in $[101,103]$ do obey these Word identities, and also use these to restore the form of 3-point amplitude with all three bosonic legs [101, 103].

\section{1 $\mathrm{D}=10$ and $\mathrm{D}=11$ spinor moving frame as spinor helicity variables}

To arrive at $\mathrm{D}=10$ spinor helicity formalism [25] used in $[26,28,29]$ to study the $10 \mathrm{D}$ SYM and type IIB supergravity amplitudes, and at its $\mathrm{D}=11$ generalization briefly presented in [30], let us consider a vector frame $\left(u_{a i}^{=}, u_{a i}^{\#}, u_{a i}^{I}\right)$ attached to a light-like 10D (11D) momentum $k_{a i}, k_{a i} k_{i}^{a}=0$, of i-th of scattered particles in such a way that (cf. (3.8))

$$
k_{i}^{a}=\rho_{i}^{\#} u_{i}^{a=} .
$$

We allow $\rho_{i}^{\#}$ to be negative, associating such with in-states of the scattering, while positive $\rho_{i}^{\#}$ are associated to out-states. This corresponds to the usual convention on that all the momenta in the amplitude are considered as, say, outgoing but the incoming particles have negative energy $k_{i}^{0}$.

The other vectors of the $i$-th frame

$$
u_{a i}^{(b)}=\left(u_{a i}^{=}, u_{a i}^{\#}, u_{a i}^{I}\right) \in \mathrm{SO}(1, D-1)
$$

are not fixed by any additional conditions except for that they form an orthogonal and normalized frame with $u_{a i}^{\bar{z}}$ (i.e. obey (3.12), (3.13)). Thus the transformations mixing $u_{a i}^{I}$ 's among themselves $(\mathrm{SO}(D-2))$ and with $u_{a i}^{\#}\left(K_{D-2}\right)$ can be considered as a kind of gauge symmetry transformations acting on the frame. In the same manner one can treat the scaling transformations of $u_{a i}^{=}$: supplemented by opposite scaling of $\rho_{(i)}^{\#}$. These leave invariant the momentum (4.1) and, when supplemented by the opposite scaling of $u_{a i}^{\#}$, can be identified with $\mathrm{SO}(1,1) \subset \mathrm{SO}(1, D-1)$ transformations leaving invariant (3.12) and, hence, the splitting (4.2).

Thus the complete set of transformations which can be used as identification relations on the class of frames defined by the only condition (4.1) (set of gauge symmetries relating equivalent vector frames) form the $[\mathrm{SO}(1,1) \otimes \mathrm{SO}(D-2)] \otimes K_{D-2}$ subgroup of $\mathrm{SO}(1, D-1)$. The frames obeying (4.1) with some light-like momentum span the coset 
$\frac{\mathrm{SO}(1, D-1)}{[\mathrm{SO}(1,1) \otimes \mathrm{SO}(D-2)] \otimes K_{D-2}}$ isomorphic to the celestial sphere of a D-dimensional observer. We would like to express this fact by writing (cf. (3.17))

$$
\left\{u_{a i}^{=}\right\} \in \frac{\mathrm{SO}(1, D-1)}{[\mathrm{SO}(1,1) \otimes \mathrm{SO}(D-2)] \otimes K_{D-2}} .
$$

Notice that, in distinction to (3.17), here we have used $\in$ symbol (rather then $=$ ), as this is more appropriate when we are speaking about a definite scattering process.

In $\mathrm{D}=10$ case we introduce the corresponding spinor frame matrix

$$
V_{\alpha i}^{(\beta)}=\left(v_{\alpha \dot{q} i}^{+}, v_{\alpha q i}^{-}\right) \in \operatorname{Spin}(1, D-1)
$$

and its inverse

$$
V_{(\beta) i}^{\alpha}=\left(\begin{array}{c}
v_{q i}^{+\alpha} \\
v_{\dot{q} i}^{-\alpha}
\end{array}\right) \in \operatorname{Spin}(1, D-1)
$$

related among themselves by (3.24) and to the above frame vectors by eqs. (3.26)-(3.29). Eqs. (3.26) imply

$$
\begin{aligned}
D=10: \quad k_{a i} \Gamma_{\alpha \beta}^{a} & =2 \rho_{i}^{\#} v_{\alpha q i}^{-} v_{\beta q i}^{-}, & \rho^{\#} v_{\dot{q} i}^{-} \tilde{\Gamma}_{a} v_{\dot{p} i}^{-}=k_{a i} \delta_{q p}, \\
k_{a i} \tilde{\Gamma}^{a \alpha \beta} & =2 \rho_{i}^{\#} v_{\dot{q} i}^{-\alpha} v_{\dot{q} i}^{-\beta}, & \rho^{\#} v_{\dot{q} i}^{-} \Gamma_{a} v_{\dot{p} i}^{-}=k_{a i} \delta_{\dot{q} \dot{p}} .
\end{aligned}
$$

Contracting suitable equations (4.6) with $v_{q i}^{-\beta}$ and $v_{\alpha \dot{q} i}{ }^{-}$and using (3.24) we easily find that these obey the massless Dirac equations (Weyl equations)

$$
k_{a i} \Gamma_{\alpha \beta}^{a} v_{\dot{q} i}^{-\beta}=0, \quad k_{a i} \tilde{\Gamma}^{a \alpha \beta} v_{\beta q i}^{-}=0 .
$$

Thus they can be identified with $\mathrm{D}=10$ spinor helicity variables of [25]

$$
\lambda_{\alpha q}=\sqrt{\rho^{\#}} v_{\alpha q}^{-}
$$

and, as we will see in a moment, with its $\mathrm{D}=11$ generalization.

The counterparts of the basic relations of the corresponding spinor helicity formalism for $D=11$ supergravity can be extracted from [38]. In this case we can also adapt 11D vector frame to a light-like momentum according to (4.1); then the spinor moving frame variables/sinorial harmonics obey the counterpart of the constraints (4.6)

$$
D=11: \quad k_{a i} \Gamma_{\alpha \beta}^{a}=2 \rho_{i}^{\#} v_{\alpha q i}^{-} v_{\beta q i}^{-}, \quad \rho^{\#} v_{q i}^{-} \tilde{\Gamma}_{a} v_{p i}^{-}=k_{a i} \delta_{q p},
$$

with $\alpha, \beta=1, \ldots, 32, q, p=1, \ldots, 16$ and $a=0,1, \ldots, 10$. Notice that in this case the counterparts of the equations in the second line of (4.6),

$$
D=11: \quad k_{a i} \tilde{\Gamma}^{a \alpha \beta}=2 \rho_{i}^{\#} v_{q i}^{-\alpha} v_{q i}^{-\beta}, \quad \rho^{\#} v_{q i}^{-} \Gamma_{a} v_{p i}^{-}=k_{a i} \delta_{q p},
$$

are equivalent to (4.9). Again, one can identify spinor harmonics with the solutions of massless Dirac equation

$$
k_{a i} \tilde{\Gamma}^{a \alpha \beta} v_{\beta q i}^{-}=0 \quad \Leftrightarrow \quad k_{a i} \Gamma_{\alpha \beta}^{a} v_{q i}^{-\beta}=0 .
$$

Hence, the 11D generalization of the 10D spinor helicity variables is given by (4.8) with $\alpha=1, \ldots, 32$ and $q=1, \ldots, 16$. 
As many equations of $10 \mathrm{D}$ and $11 \mathrm{D}$ spinor frame/spinor helicity formalism differs formally by just replacing dotted indices $\dot{q}, \dot{p}$ by undotted $q, p$, and by changing the range of the values of the indices, below, when this cannot lead to confusion, we will not duplicate the equations writing them separately for $\mathrm{D}=10$ and $\mathrm{D}=11$ cases, but rather write single equation assuming that for $\mathrm{D}=11$ case $\dot{q}=q$, etc.

Polarization spinor of the $\mathrm{D}=10$ and $\mathrm{D}=11$ fermionic fields can be associated with the element of the inverse $\mathrm{D}=10$ and $\mathrm{D}=11$ spinor frame matrix,

$$
\lambda_{\dot{q} i}^{\alpha}=\sqrt{\rho^{\#}} v_{\dot{q} i}^{-\alpha} .
$$

For $D=11, \dot{q}=q$ and eq. (4.12), $\lambda_{q i}^{\alpha}=\sqrt{\rho^{\#}} v_{q i}^{-\alpha}$, is equivalent to (4.8) due to (3.25).

Notice that $v_{\alpha \dot{q} i}^{+}$and $v_{q i}^{+\alpha}$ are not included explicitly in the definition of momentum and polarization variables and in this sense can be treated as constrained reference spinors restricted by the requirement to form, together with $v_{\alpha q i}^{-}$and $v_{\dot{q} i}^{-\alpha}$, the $\operatorname{Spin}(1, D-1)$ valued matrix (4.4) and its inverse (4.5). The freedom in the definition of $v_{\alpha \dot{q} i}^{+}$includes the $K_{D-2}$ transformations

$$
v_{\alpha \dot{q} i}^{+} \mapsto v_{\alpha \dot{q} i}^{+}+\frac{1}{2} K_{i}^{\# I} v_{\alpha p i}^{-} \gamma_{p \dot{q}}^{I}, \quad v_{\alpha q i}^{-} \mapsto v_{\alpha q i}^{-}
$$

as well as the natural action of $\operatorname{Spin}(D-2)$ on $q, p$ and $\dot{q}, \dot{p}$ indices and scaling, supplemented by an opposite scaling of $v_{\alpha q i}^{-}$. With the same line of argument as presented for the vector frame when arriving at (4.3), we arrive at

$$
\left\{v_{\alpha q i}^{-}\right\} \in \frac{\operatorname{Spin}(1, D-1)}{[\mathrm{SO}(1,1) \otimes \operatorname{Spin}(D-2)] \otimes K_{D-2}}=\mathbb{S}^{D-2} .
$$

The simplest application of our spinor frame form of the spinor helicity formalism is to write the solution of the momentum representation of the linearized equations of motion of 10D SYM and 11D SUGRA multiplets.

\section{2 $\mathrm{D}=10 \mathrm{SYM}$ multiplet in spinor helicity formalism}

As, according to (4.12), polarization of a 10D spinor field can be described by 8 constrained spinors from the inverse spinor frame matrix (4.5), $v_{\dot{q}}^{-\alpha}$ which obey (4.7), the general solution of linearized massless Dirac equation reads

$$
D=10: \quad \chi^{\alpha}=v_{\dot{q}}^{-\alpha} \psi_{\dot{q}}, \quad \alpha=1, \ldots, 16, \quad \dot{q}=1, \ldots, 8,
$$

and the superpartner of the gauge field is characterized by a fermionic $\mathrm{SO}(8)$ c-spinor field $\psi_{\dot{q}}$.

Polarization vector of a gauge field can be identified with the spacelike vector $u_{a}^{I}$ of the frame (4.2) adapted to the light-like momentum $k_{a}$ through (4.1) (cf. [25]). Then the basic solutions of the linearized YM equations can be written as $F_{a b}{ }^{I}=k_{[a} u_{b]}{ }^{I}$ and the general solution

$$
D=10: \quad F_{a b}=k_{[a} u_{b]}^{I} w^{I}, \quad k_{a}=\rho^{\#} u_{a}^{=}, \quad a=0,1, \ldots, 9, \quad I=1, \ldots, 8,
$$

is characterized by an $\mathrm{SO}(8)$ vector $w^{I}$. 
When the formalism is applied to external particles of scattering amplitudes, coefficients in the above expressions for bosonic and fermionic fields, the bosonic $w^{I}$ and fermionic $\psi_{q}$, can be taken to be dependent on the on-shell momentum $k_{a}$; in our formalism this implies the dependence on the constrained spinors $v_{\alpha q}^{-}(4.14)$ and density $\rho^{\#}$ (see (4.6)). Alternatively, we can replace $\rho^{\#}$ by its conjugate coordinate and consider the field $w^{I}=w^{I}\left(x^{=}, v_{q}^{-}\right)$and $\psi_{q}=\psi_{q}\left(x^{=}, v_{q}^{-}\right)$on the nine-dimensional space $\mathbb{R} \otimes \mathbb{S}^{8}$. We will see below that supersymmetry acts on these $9 \mathrm{~d}$ fields as

$$
\delta_{\epsilon} w^{I}\left(x^{=}, v_{q}^{-}\right)=2 i \epsilon^{-q} \gamma_{q \dot{q}}^{I} \psi_{\dot{q}}\left(x^{=}, v_{q}^{-}\right), \quad \delta_{\epsilon} \psi_{\dot{q}}\left(x^{=}, v_{q}^{-}\right)=\epsilon^{-q} \gamma_{q \dot{q}}^{I} \partial_{=} w^{I}\left(x^{=}, v_{q}^{-}\right),
$$

where the fermionic $\mathrm{SO}(8)$ s-spinors $\epsilon^{-q}$ are expressed through the constant fermionic spinor parameter of rigid $10 \mathrm{D} \mathcal{N}=1$ supersymmetry, $\epsilon^{\alpha}$, by

$$
\epsilon^{-q}=\epsilon^{\alpha} v_{\alpha q}^{-}
$$

In $\mathrm{D}=4$ the field describing physical degrees of freedom of the maximal SYM multiplet, appear in the decomposition of an on-shell superfield on fermionic coordinate, (2.24). The natural question is whether such an on-shell superfield may exist in the case of D=10 SYM. As we will see below, the answer on this question is affirmative.

\subsection{D SUGRA in spinor helicity formalism}

In $\mathrm{D}=11$ it is convenient to begin with the solution of the linearized equations for the on-shell field strength $F_{a b c d}$ of the 3-form gauge field. By analogy with the above described $10 \mathrm{D}$ gauge field strength, the solution can be expressed in terms of $11 \mathrm{D} u_{a}^{I}, F_{a b c d}^{I J K}=$ $k_{[a} u_{b}^{I} u_{c}^{J} u_{d]}^{K}$, so that the generic linearized field strength

$$
D=11: \quad F_{a b c d}=k_{[a} u_{b}^{I} u_{c}^{J} u_{d]}^{K} A_{I J K}, \quad a=0,1, \ldots, 10, \quad I=1, \ldots, 9,
$$

is expressed in terms of an antisymmetric $\mathrm{SO}(9)$ tensor $A_{I J K}$. Its superpartners, $\gamma$-traceless $\Psi_{I q}$ and symmetric traceless $h_{I J}$, are used to make a decomposition of linearized 11D graviton and gravitino fields,

$$
\begin{aligned}
& D=11: \quad \psi_{a b}^{\alpha}=k_{[a} u_{b]}^{I} v_{q}^{-\alpha} \Psi_{I q}, \quad \gamma_{q p}^{I} \Psi_{I p}=0, \\
& h_{a b}=u_{(a}^{I} u_{b)}^{J} h_{I J}, \quad h_{I I}=0 .
\end{aligned}
$$

The linearised on-shell Riemann tensor reads

$$
R_{a b}^{c d}=k_{[a} u_{b]}^{I} k^{[c} u^{d] J} h_{I J} .
$$

It is easy to check that this obeys the characteristic identities $R_{[a b c]}{ }^{d}=0, R_{a b c d}=R_{c d a b}$ as well as the linearized Einstein equations $R_{a b}{ }^{c b}=0$.

Again, it is natural to expect that the above fields appear as independent components of a constrained $d=1, \mathcal{N}=16$ on-shell superfield. As we will show below, this is indeed the case. 


\subsection{D amplitudes in spinor helicity formalism, supersymmetry and Ward identities}

The tree amplitudes of 10D SYM should depend on the light-like momentum and polarization vectors or spinors of scattered particles and can carry some nontrivial representations of a small group, in particular of its $\mathrm{SO}(8)$ subgroup. In the light of the above discussion, this implies that the $n$-point tree amplitudes, a $D=10$ counterpart of (2.6), should depend on $n$ sets of spinor moving frame variables $v_{\alpha q(i)}^{-}$and $n$ 'energies' $\rho_{(i)}^{\#}$, corresponding to each of the scattered particles; it can also carry the $\mathrm{SO}(8)$ polarization indices for each of the scattered particles. The superamplitudes, counterparts of $4 \mathrm{D}$ (2.23), shall also depend on fermionic variables, but in this section we would like to discuss briefly a 'component' (rather than superfield) approach to SYM amplitudes.

To understand the structure and properties of the supersymmetry transformations of the amplitudes, it is convenient to consider 16-parametric rigid supersymmetry as a superposition of $n$ 8-parametric SUSY transformations defined such that $i$-th 'item supersymmetries' with parameters $\epsilon_{i}^{-q}$ acts on the variables and/or indices corresponding to $i$-th scattered particle. The true supersymmetry transformations will involve all the item supersymmetries with parameters expressed in terms of the constant fermionic spinor of rigid supersymmetry and $i$-th spinor frame variables by

$$
\epsilon_{i}^{-q}=\epsilon^{\alpha} v_{\alpha q i}^{-}
$$

The structure of the supersymmetry transformation relating on-shell fields of the SYM supermultiplet, (4.17), suggests that the supermultiplet with respect to $i-t h$ 'item supersymmetry' is formed by two amplitudes which differs by only one of their $n$ polarization indices, namely by the index corresponding to $i$-th scattered particle, and that this index can be either of $\mathbf{8 v}$ or of $\mathbf{8} \mathbf{c}$ representation, ${ }^{10}$

$$
\begin{gathered}
\mathcal{A}_{\ldots I_{i} \ldots}^{(n)}\left(k_{1}, \ldots, k_{i}, \ldots, k_{n}\right)=\mathcal{A}_{\ldots I_{i} \ldots}^{(n)}\left(\left\{\rho_{j}^{\#}, v_{q j}^{-}\right\}\right) \\
\text {and } \\
\mathcal{A}_{\ldots \dot{q}_{l} \ldots}^{(n)}\left(k_{1}, \ldots, k_{i}, \ldots, k_{n}\right)=\mathcal{A}_{\ldots \dot{q}_{i} \ldots}^{(n)}\left(\left\{\rho_{j}^{\#}\right\} ;\left\{v_{q j}^{-}\right\}\right) .
\end{gathered}
$$

Of course, for any particular combinations of the indices denoted by multidots, either $\mathcal{A}_{\ldots I_{i} \ldots}^{(n)}$ or $\mathcal{A}_{\ldots \dot{q}_{i} \ldots}^{(n)}$ vanishes by the fermionic number preservation (see below for more details). Nevertheless it is instructive first to write the generic $i$ - th SUSY transformations with Grassmann parameter $\epsilon_{i}^{-q}$ as they are suggested by transformations (4.17) of the on-shell SYM fields $\left(w^{I}, \psi_{\dot{q}}\right)$ :

$$
\begin{aligned}
& \delta_{\epsilon_{i}} \mathcal{A}_{\ldots I_{i} \ldots}^{(n)}\left(\ldots, k_{i}, \ldots\right)=2 i(-)^{\Sigma_{i}} \epsilon_{i}^{-q} \gamma_{q \dot{q}_{i}}^{I_{i}} \mathcal{A}_{\ldots \dot{q}_{i} \ldots}^{(n)}\left(\ldots, k_{l}, \ldots\right) \\
& \delta_{\epsilon_{i}} \mathcal{A}_{\ldots \dot{q}_{i} \ldots}^{(n)}\left(\ldots, k_{i}, \ldots\right)=i(-)^{\Sigma_{i}} \rho_{i}^{\#} \epsilon_{i}^{-q} \gamma_{q \dot{q}_{i}}^{I_{i}} \mathcal{A}_{\ldots I_{i} \ldots}^{(n)}\left(\ldots, k_{i}, \ldots\right)
\end{aligned}
$$

\footnotetext{
${ }^{10}$ The third, 8s representation is singled out by that, in our notation, its indeex is carfried by the spinor frame variables $v_{\alpha q i}^{-}$related to the light-like momentum $k_{a i}$ of $i$-th particle by (4.6).
} 
The sign factor $(-)^{\Sigma_{i}}$ in (4.25) will be specified below (see also eq. (6.11)). ${ }^{11}$ The complete expressions (4.25) are also valid for the case of coefficients in the decomposition of superamplitudes on fermionic coordinate corresponding to one of $n$ scattered particles.

To write the complete form of the rigid supersymmetry transformations of the amplitudes, it is convenient to introduce a cumulative index

$$
Q_{i}=\left(I_{i}, \dot{q}_{i}\right), \quad I_{i}=1, \ldots, 8, \quad \dot{q}_{i}=1, \ldots, 8
$$

allowing to describe all the amplitudes (4.24) by the universal expression

$$
\mathcal{A}_{Q_{1} \ldots Q_{n}}^{(n)}\left(\rho_{1}^{\#}, v_{\alpha q 1}^{-} ; \ldots ; \rho_{n}^{\#}, v_{\alpha q n}^{-}\right)=: \mathcal{A}_{\ldots Q_{i} \ldots}^{(n)}\left(\ldots ; \rho_{i}^{\#}, v_{\alpha q i}^{-} ; \ldots\right) .
$$

Formally, the rigid 10D supersymmetry acts on the amplitudes by

$$
\begin{aligned}
& \delta_{\epsilon} \mathcal{A}_{Q_{1} \ldots Q_{n}}^{(n)}\left(\rho_{1}^{\#}, v_{\alpha q 1}^{-} ; \ldots ; \rho_{n}^{\#}, v_{\alpha q n}^{-}\right)= \\
& \quad=\epsilon^{\alpha} \sum_{i=1}^{n}(-)^{\Sigma_{i}} v_{\alpha q_{i}}^{-} \Delta_{q_{i} Q_{i} Q_{i}^{\prime}} \mathcal{A}_{Q_{1} \ldots Q_{i}^{\prime} \ldots Q_{n}}^{(n)}\left(\rho_{1}^{\#}, v_{\alpha q 1}^{-} ; \ldots ; \rho_{n}^{\#}, v_{\alpha q n}^{-}\right),
\end{aligned}
$$

where $\Delta_{q_{i} Q_{i} Q_{i}^{\prime}}=\Delta_{q_{i} Q_{i} Q_{i}^{\prime}}\left(\rho_{i}^{\#}\right)$ are expressed through the $\mathrm{SO}(8)$ Klebsh-Gordan coefficients $\gamma_{q \dot{q}}^{I}$ by

$$
\Delta_{q_{i} Q_{i} Q_{i}^{\prime}}=i \gamma_{q_{i} \dot{q}_{i}}^{I_{i}}\left(2 \delta_{Q_{i}}{ }^{I_{i}} \delta_{Q_{i}^{\prime} \dot{q}_{i}}+\rho_{i}^{\#} \delta_{Q_{i} \dot{q}_{i}} \delta_{Q_{i}^{\prime}}{ }^{I_{i}}\right)
$$

and $\Sigma_{i}$ counts the number of fermionic indices $\dot{q}_{j}$ among $Q_{j}$ 's with $1 \leq j<i$.

Now let us recall that the preservation of the fermionic number requires vanishing of all the amplitudes describing scattering of an odd number of fermions (when counting both incoming and outgoing particle). Then their supersymmetry transformations should also vanish. This implies, firstly, that all the (potentially) nonvanishing amplitudes, describing the scattering of an even number of fermionic and some number of bosonic particles, are supersymmetric invariant

$$
\delta_{\epsilon} \mathcal{A}_{Q_{1} \ldots Q_{n}}^{(n)}\left(\rho_{1}^{\#}, v_{\alpha q 1}^{-} ; \ldots ; \rho_{n}^{\#}, v_{\alpha q n}^{-}\right)=0
$$

and, secondly, that they obey the following supersymmetric Ward identities ${ }^{12}$

$$
\sum_{i=1}^{n}(-)^{\Sigma_{i}} v_{\alpha q_{i}}^{-} \Delta_{q_{i} Q_{i} Q_{i}^{\prime}} \mathcal{A}_{Q_{1} \ldots Q_{i}^{\prime} \ldots Q_{n}}^{(n)}\left(\rho_{1}^{\#}, v_{\alpha q 1}^{-} ; \ldots ; \rho_{i}^{\#}, v_{\alpha q i}^{-} ; \ldots ; \rho_{n}^{\#}, v_{\alpha q n}^{-}\right)=0 .
$$

As an example, let us write the explicit form of the Ward identities for 3-point amplitudes:

$$
\begin{gathered}
\rho_{1}^{\#} v_{\alpha q 1}^{-} \gamma_{q \dot{q}_{1}}^{I_{1}} \mathcal{A}_{I_{1} \dot{q}_{2} \dot{q}_{3}}^{(3)}-\rho_{2}^{\#} v_{\alpha q 2}^{-} \gamma_{q \dot{q}_{2}}^{I_{2}} \mathcal{A}_{\dot{q}_{1} I_{2} \dot{q}_{3}}^{(3)}+\rho_{3}^{\#} v_{\alpha q 3}^{-} \gamma_{q \dot{q}_{3}}^{I_{3}} \mathcal{A}_{\dot{q}_{1} \dot{q}_{2} I_{3}}^{(3)}=0, \\
2 v_{\alpha q 1}^{-} \gamma_{q_{\dot{q}}}^{I_{1}} \mathcal{A}_{\dot{q}_{1} I_{2} \dot{q}_{3}}^{(3)}+2 v_{\alpha q 2}^{-} \gamma_{q \dot{q}_{2}}^{I_{2}} \mathcal{A}_{I_{1} \dot{q}_{2} \dot{q}_{3}}^{(3)}+\rho_{3}^{\#} v_{\alpha q 3}^{-} \gamma_{q \dot{q}_{3}}^{I_{3}} \mathcal{A}_{I_{1} I_{2} I_{3}}^{(3)}=0 .
\end{gathered}
$$

\footnotetext{
${ }^{11}$ Here for shortness we have written the argument $k_{i}$ instead of $\rho_{i}^{\#}, v_{\alpha q i}^{-}$. Notice also that $\partial_{=}$in the second equation of (4.17) is replaced by $i \rho_{i}^{\#}$ in (4.25) (momentum versus coordinate representation).

${ }^{12}$ Of course, eqs. (4.31) are nontrivial only when the amplitudes carry the even number of fermionic indices, but we do not feel necessary to stress this in the formulae as otherwise they are trivially satisfied.
} 
These could be used to find the structure of the 3-point amplitudes of 10D SYM (see [103]). Below we will present a specific parametrization of spinor frames (special gauge fixed on spinor frame variables) which is especially useful for addressing such type of problems.

But before we would like to develop a constrained superamplitude formalism, based on superfield generalization of the above amplitudes. These superamplitudes can be considered as multi-particle generalizations of the constrained on-shell superfield description of the linearized SYM multiplet, which, as we will show, can be obtained from superparticle quantization.

We conclude this section by a brief description of the 11D amplitudes and the supersymmetric Ward identities for them. Their similarity with 10D counterparts allows to reduce this description to specification of the cumulative indices and $\Delta$-symbols in (4.27), (4.29), (4.30) and (4.31).

\subsection{Supersymmetric Ward identities for 11D SUGRA amplitudes}

The superamplitudes of 11D supergravity can be described by formula (4.27) with 11D spinor frame variables $v_{\alpha q i}^{-}$and cumulative indices

$$
Q=([I J K],((I J)), I q), \quad I, J, K=1, \ldots, 8,9, \quad q=1, \ldots, 16,
$$

including antisymmetric combination of $\mathrm{SO}(9)$ vector indices $[I J K]$, symmetric traceless combination of two $\mathrm{SO}(9)$ vector indices $((I J))$ [to lighten equation we will sometimes write this with one set of brackets, as $(I J)]$ and $\gamma$-traceless combination of the vector and spinor indices of $\mathrm{SO}(9), I q$.

Then the supersymmetric Ward identities for these amplitudes will have the form of eq. (4.31) with the following components of $\Delta_{q_{i} Q_{i} Q_{i}^{\prime}}=\Delta_{q_{i} Q_{i} Q_{i}^{\prime}}\left(\rho_{i}^{\#}\right)$ :

$$
\begin{aligned}
\Delta_{q[I J K] L p} & =i \delta^{L[I} \gamma_{q p}^{J K]}, & \Delta_{q((I J)) K p} & =i \delta^{K((I} \gamma_{q p)}^{J)}, \\
\Delta_{q J p((K L))} & =2 i \rho_{i}^{\#} \delta^{K((I} \gamma_{q p}^{J))}, & \Delta_{q J p[K L M]} & =\frac{i}{18} \rho_{(i)}^{\#}\left(\gamma_{q p}^{J K L M}+6 \delta^{J[K} \gamma_{q p}^{L M]}\right) .
\end{aligned}
$$

\section{$5 \mathrm{D}=10$ and $\mathrm{D}=11$ on-shell superfields from quantization of massless superparticle in analytical basis of Lorentz harmonic superspace}

In this section we show how the above descriptions of linearized 10D SYM and 11D SUGRA supermultiplet appear, in their superfield form, in quantization of $\mathrm{D}=10$ and $\mathrm{D}=11$ massless superparticle models. The reader not interested in quantization procedure may omit the first four subsections and pass directly to subsection 5.5.2, using subsection 5.5.1 just for notation.

\subsection{Changing variables in $\mathrm{D}=\mathbf{1 0 , 1 1}$ massless superparticle action}

As we discussed in section 2.4, 4D on-shell superfields can be obtained from quantization of $\mathrm{D}=4$ massless superparticle mechanics reformulated in terms of bosonic spinor and their conjugate variables. The original Ferber-Shirafuji action (2.33) can be written in terms of these variables, $(2.34)$, by using the Leibnitz rule to move $\partial_{\tau}$ derivatives. 
Let us try to do the same in the analogous spinor moving frame action for $\mathrm{D}=10$ and $\mathrm{D}=11$ massless superparticle, which is given in (3.31) with the range of indices described in (3.21) and (3.22), respectively. One can easily arrive at

$$
S=\int d \tau \rho^{\#}\left(\partial_{\tau} X^{=}-2 i \partial_{\tau} \theta_{q}{ }^{-} \theta_{q}{ }^{-}-\partial_{\tau} v_{\alpha q}^{-}(\ldots)^{\alpha q}\right)
$$

where

$$
X^{=}=X^{a} u_{a}^{=}, \quad \theta_{q}^{-}=\theta^{\alpha} v_{\alpha q}^{-}
$$

which can be associated with coordinates of the so-called analytical coordinate basis of Lorentz harmonic superspace, which we describe in the next section 5.2, and multidots denote $\frac{1}{4} X^{a} \tilde{\Gamma}_{a}^{\alpha \beta} v_{\beta q}{ }^{-}-i \theta^{\alpha} \theta^{\beta} v_{\beta q}{ }^{-}$. Actually this expression can also be rewritten in terms of coordinates of the analytical basis of the Lorentz harmonic superspace. However, to perform this in a brief and clear manner, we need first to clarify the structure of the derivative of spinor moving frame variable, $\partial_{\tau} v_{\alpha q}^{-}$entering the third term in the integrand of (5.1), or more generally of the differential of this variable $d v_{\alpha q}^{-}$. In section 5.3 we will show how to express these in term of Cartan forms of the Lorenz group.

\subsection{Analitical basis of Lorenz harmonic superspace and its invariant sub-superspaces}

As we have already mentioned, the spinor moving frame formulation of massless superparticle can be considered as a dynamical system in an enlarged superspace called Lorentz harmonic superspace with the bosonic body given by the direct product of D-dimensional Minkowski space and 2(D-2)-dimensional coset of the D-dimensional Lorentz group, $\mathbb{R}^{1,(D-1)} \times \frac{\mathrm{SO}(1, D-1)}{\mathrm{SO}(1,1) \otimes \mathrm{SO}(D-2)}$.

The set of coordinates of Lorentz harmonic superspace includes, in addition to the usual bosonic D-vector and fermionic spinor coordinates, also Lorentz harmonics (spinor moving frame variables) $v_{\alpha q}^{-}, v_{\alpha \dot{q}}^{+}$, 'parametrizing' (as a kind of homogeneous coordinates) a non-compact coset $\frac{\mathrm{SO}(1, D-1)}{\mathrm{SO}(1,1) \otimes \mathrm{SO}(D-2)}$,

$$
\mathcal{Z}^{\mathcal{M}}=\left(X^{a}, \theta^{\alpha}, v_{\alpha q}^{-}, v_{\alpha \dot{q}}^{+}\right)
$$

(the ranges of values of indices for $\mathrm{D}=10$ and $\mathrm{D}=11$ cases are given in (3.21) and (3.22), respectively). Notice that these Lorentz harmonic variables are also appropriate for the description of (super)string [39-41]. ${ }^{13}$

\footnotetext{
${ }^{13}$ The spinor moving frame formulations of super-p-branes [39, 40, 44] use the Lorentz harmonics parametrizing the noncompact cosets $\frac{\mathrm{SO}(1, D-1)}{\mathrm{SO}(1, p) \otimes \mathrm{SO}(D-p-1)}$ with an appropriate values of $\mathrm{D}$; e.g. for superstring we can consider $D=3,4,6,10$. The spinor moving frame formulation of superparticle [33, 36-38] uses $v_{\alpha q}^{-}$variables parametrizing, modulo gauge symmetries, a compact coset [31,32, 35]. However, when such a formulation is treated as superparticle in Lorentz harmonic superspace, it is convenient to have also $v_{\alpha \dot{q}}^{+}$, the counterpart of reference spinor, as a superspace coordinate (see [85] for $\mathrm{D}=4$ model).
} 
The coordinate basis (5.3) is called 'central basis'. Besides this we can define an analytical basis with coordinates (cf. $[78,79]$ and, in a more general perspective, [82-84])

$$
\begin{aligned}
\mathcal{Z} \frac{\mathcal{M}}{A} & =\left(X^{\#}, X^{=}, X^{I}, \theta_{\dot{q}}^{+}, \theta_{q}^{-}, v_{\alpha q}^{-}, v_{\alpha \dot{q}}^{+}\right), \\
X^{=}: & =X^{a} u_{a}^{=}, \quad X^{\#}:=X^{a} u_{a}^{\#}, \quad X^{I}:=X^{a} u_{a}^{I}+i \theta_{q}^{-} \gamma_{q \dot{q}}^{I} \theta_{\dot{q}}^{+}, \\
\theta_{\dot{q}}^{+} & =\theta^{\alpha} v_{\alpha \dot{q}}^{+}, \quad \theta_{q}^{-}=\theta^{\alpha} v_{\alpha q}{ }^{-} .
\end{aligned}
$$

Notice the nontrivial fermionic bilinear contributions in the definition of $X^{I}$ in (5.5). They are designed in such a way, that the superspace supersymmetry transformations

$$
\delta_{\varepsilon} X^{a}=i \theta \Gamma^{a} \varepsilon, \quad \delta_{\varepsilon} \theta^{\alpha}=\varepsilon^{\alpha}
$$

can be closed on smaller set of coordinates

$$
\tilde{\zeta}^{\tilde{\mathcal{M}}}=\left(X^{=}, X^{I}, \theta_{q}^{-}, v_{\alpha q}^{-}, v_{\alpha \dot{q}}^{+}\right) .
$$

These parametrize an invariant sub-superspace of the Lorentz-harmonic superspace which is called analytic (sub)superspace. The supersymmetry transformations of the analytic superspace coordinates (5.8) read

$$
\delta_{\varepsilon} X^{=}=2 i \theta_{q}^{-} \varepsilon^{-q}, \quad \delta_{\varepsilon} X^{I}=2 i \theta_{q}^{-} \gamma_{q \dot{p}}^{I} \varepsilon^{+\dot{p}}, \quad \delta_{\varepsilon} \theta_{q}^{-}=\varepsilon^{-q}, \quad \delta_{\varepsilon} v_{\alpha \dot{q}}{ }^{-}=0=\delta_{\varepsilon} v_{\alpha q}{ }^{+},
$$

where (cf. (4.18))

$$
\varepsilon^{-q}=\varepsilon^{\alpha} v_{\alpha q}^{-}, \quad \varepsilon^{+\dot{q}}=\varepsilon^{\alpha} v_{\alpha \dot{q}}^{+}
$$

Actually the smaller superspace with coordinates

$$
\zeta^{\mathcal{M}}=\left(X^{=}, \theta_{q}^{-}, v_{\alpha q}^{-}, v_{\alpha \dot{q}}^{+}\right)
$$

is also invariant under (5.9),

$$
\delta_{\varepsilon} X^{=}=i \theta_{q}^{-} \varepsilon^{-q}, \quad \delta_{\varepsilon} \theta_{q}^{-}=\varepsilon^{-q}, \quad \delta_{\varepsilon} v_{\alpha q}{ }^{-}=0=\delta_{\varepsilon} v_{\alpha \dot{q}}{ }^{+} .
$$

One can observe however that only a half of the supersymmetries acts efficiently on this minimal analytic (sub)superspace.

Below we will see that the minimal superspaces (5.11) are sufficient to provide the arena for on-shell superfields describing 10D SYM and linearized 11D supergravity multiplets. Then, $n$-point superamplitudes will be defined on the direct product of $n$ copies of minimal analytic superspaces (5.11). In contrast, to write the massless superparticle actions the coordinates $X^{I}$ are also needed, so that the superparticle can be considered as a particle in the 'non-minimal' analytic superspace (5.8).

Another important remark is related to the fact that the massless superparticle action (3.31) contains the coordinate functions $v_{\alpha q}^{-}$, but not $v_{\alpha \dot{q}}^{+}$. As it is also invariant under $\mathrm{SO}(1,1) \otimes \mathrm{SO}(D-2)$ symmetry, using this as identification relation on the set of $v_{\alpha q}^{-}$, we concluded, following [31,32], that these constrained variables parametrize the compact coset isomorphic to the celestial sphere (see (4.14) and the descussion above it). 
This statement can be reformulated by considering the superparticle action as apparently depending on the complete set of Lorentz harmonics $v_{\alpha q}^{-}, v_{\alpha \dot{q}}^{+}$parametrizing the non-compact coset $\frac{\mathrm{SO}(1, D-1)}{\mathrm{SO}(1,1) \otimes \mathrm{SO}(D-2)}$, i.e. as an action of superparticle in Lorentz harmonic superspace (5.3), which possesses the gauge symmetry under $K_{D-2} \cdot{ }^{14}$ The role of $K_{D-2}$ symmetry, which completes $\mathrm{SO}(1,1) \otimes \mathrm{SO}(D-2)$ till Borel subgroup of $\mathrm{SO}(1, D-1)$, is to make 'unphysical' (pure gauge) the complementary element of $\operatorname{Spin}(1, \mathrm{D}-1)$ valued matrix, $v_{\alpha \dot{q}}^{+}\left(v_{\alpha q}^{+}\right.$in $11 \mathrm{D}$ case $)$. Such a point of view is reflected by stating that homogeneous coordinates of the coset $\frac{\mathrm{SO}(1, D-1)}{[\mathrm{SO}(1,1) \otimes \mathrm{SO}(D-2)] \otimes K_{D-2}}=\mathbb{S}^{D-2}$ (i.e. of celestial sphere) are given by $\left(v_{\alpha q}^{-}, v_{\alpha \dot{q}}^{+}\right)$, i.e. by writing

$$
\left\{\left(v_{\alpha q}^{-}, v_{\alpha \dot{q}}^{+}\right)\right\}=\frac{\operatorname{Spin}(1, D-1)}{[\mathrm{SO}(1,1) \otimes \operatorname{Spin}(D-2)] \otimes K_{D-2}}=\mathbb{S}^{D-2},
$$

instead of (4.14) and

$$
\left\{\left(u_{a}^{=}, u_{a}^{\#}, u_{a}^{I}\right)\right\}=\frac{\mathrm{SO}(1, D-1)}{[\mathrm{SO}(1,1) \otimes \mathrm{SO}(D-2)] \otimes K_{D-2}}=\mathbb{S}^{D-2},
$$

instead of (3.16).

This point of view is actually preferable for our discussion in this section.

\subsection{Cartan forms and derivatives of the spinor moving frame variables}

To understand the structure of the last term in the analytical basis form of the massless superparticle action (5.1), we need to describe the structure of derivative of the spinor moving frame variables. This is not apparent as these variables are strongly constrained by eqs. (3.26)-(3.30) the set of which is equivalent to (3.20). However, actually the problem can be easily solved by using clear group theoretical meaning of the moving frame and spinor moving frame variables. Indeed, as these can be understood as elements of Lorentz $\mathrm{SO}(1, D-1)$ (Spin(1,D - 1)) group valued matrix, their differential (and variation) should belong to the space (co-)tangent to the Lorentz group which is isomorphic (actually dual) to the Lie algebra $s o(1, D-1)$. A basis of this space is provided by the $\mathrm{SO}(1, D-1)$ Cartan forms. We briefly describe these in the next subsections referring to $[37,38]$ for more details.

\subsubsection{Cartan forms and vector harmonics}

The derivatives of the moving frame variables (vector harmonics) are expressed in terms of $\mathrm{SO}(1, D-1)$ Cartan forms. Thier set can be split on subsets of $(D-2)$ 1-forms $\Omega^{=I}:=u_{a}^{=} d u^{a I}$ which provide a covariant basis of the space cotangent to the coset $\frac{\mathrm{SO}(1, D-1)}{[\mathrm{SO}(1,1) \otimes \mathrm{SO}(D-2)] \otimes K_{D-2}}=\mathbb{S}^{D-2}$, of $(D-2)$ forms $\Omega^{\# I}:=u_{a}^{\#} d u^{a I}$ dual to $K_{D-2}$ generators, and of the forms $\Omega^{(0)}:=\frac{1}{4} u_{a}^{=} d u^{a \#}$ and $\Omega^{I J}:=u_{a}^{I} d u^{a J}$ which have the properties of

\footnotetext{
${ }^{14}$ See $[85]$ for $\mathrm{D}=4$ case and also earlier $[78,79]$ where the 'light-cone superspace' with additional vector frame coordinates $u_{a}^{=}, u_{a}^{\#}, u_{a}^{I}$ was introduced. One more equivalent form of the same statement is that the action depends on $v_{\alpha q}^{-}, v_{\alpha \dot{q}}^{+}$which are constrained by $(3.20)$, and has the gauge symmetry under $[\operatorname{SO}(1,1) \otimes$ $\mathrm{SO}(D-2)] \otimes K_{D-2}$.
} 
connection under $\mathrm{SO}(1,1)$ and $\mathrm{SO}(D-2)$ gauge transformations. This definition of Cartan forms can be encoded in expressions for the $\mathrm{SO}(1,1) \otimes \mathrm{SO}(D-2)$ covariant derivatives of the moving frame variables

$$
\begin{aligned}
D u_{a}^{=} & :=d u_{a}^{=}+2 u_{a}^{=} \Omega^{(0)}=u_{a}^{I} \Omega^{=I}, \\
D u_{a}^{\#} & :=d u_{a}^{\#}-2 u_{a}^{\#} \Omega^{(0)}=u_{a}^{I} \Omega^{\# I}, \\
D u_{a}^{I} & :=d u_{a}^{I}+u_{a}^{J} \Omega^{J I}=\frac{1}{2} u_{a}^{\#} \Omega^{=I}+\frac{1}{2} u_{a}^{=} \Omega^{\# I} .
\end{aligned}
$$

These expressions automatically take into account the constrained nature of the vector harmonics, i.e. they guaranty the preservation of the set of constraints (3.11), (3.12) and (3.13) (equivalent to (3.10)) under the action of differential $d$.

The selfconsistency conditions for (5.15), (5.16) and (5.17) are given by Ricci identities

$$
D D u_{a}^{=}=2 u_{a}^{=} d \Omega^{(0)}, \quad D D u_{a}^{\#}=-2 u_{a}^{\#} d \Omega^{(0)}, \quad D D u_{a}^{I}=u_{a}^{J} G^{J I}
$$

which are equivalent to the Maurer-Cartan equations of the $\mathrm{SO}(1, D-1)$ group

$$
\begin{aligned}
D \Omega^{=I} & :=d \Omega^{=I}+2 \Omega^{=I} \wedge \Omega^{(0)}+\Omega^{I J} \wedge \Omega^{=J}=0, \\
D \Omega^{\# I} & :=d \Omega^{\# I}-2 \Omega^{\# I} \wedge \Omega^{(0)}+\Omega^{I J} \wedge \Omega^{\# J}=0, \\
d \Omega^{(0)} & =\frac{1}{4} \Omega^{=I} \wedge \Omega^{\# I}, \\
G^{I J} & :=d \Omega^{I J}+\Omega^{I K} \wedge \Omega^{K J}=-\Omega^{=[I} \wedge \Omega^{\# J]} .
\end{aligned}
$$

\subsubsection{Derivatives of spinorial harmonics}

$\operatorname{Spin}(1, D-1)$, the double covering of the Lorentz group $\mathrm{SO}(1, D-1)$, is locally isomorphic to it. Hence the tangent space to $\operatorname{Spin}(1, D-1)$ is isomorphic to tangent space to $\mathrm{SO}(1, D-$ 1). Hence the covariant derivatives of $\mathrm{D}=10$ and $\mathrm{D}=11$ spinor harmonics are also expressed in terms of the above Cartan forms. One finds

$$
\begin{aligned}
& D v_{\alpha q}^{-}:=d v_{\alpha q}^{-}+\Omega^{(0)} v_{\alpha q}^{-}+\frac{1}{4} \Omega^{I J} v_{\alpha p}^{-} \gamma_{p q}^{I J}=\frac{1}{2} \Omega^{=I} \gamma_{q \dot{q}}^{I} v_{\alpha \dot{q}}^{+}, \\
& D v_{\alpha \dot{q}}^{+}:=d v_{\alpha \dot{q}}^{+}-\Omega^{(0)} v_{\alpha \dot{q}}^{+}+\frac{1}{4} \Omega^{I J} v_{\alpha \dot{p}}^{+} \tilde{\gamma}_{\dot{p} \dot{q}}^{I J}=\frac{1}{2} v_{\alpha q}^{-} \Omega^{\# I} \gamma_{q \dot{q}}^{I} .
\end{aligned}
$$

For the components of the inverse spinor moving frame matrix we find

$$
\begin{aligned}
& D v_{\dot{q}}^{-\alpha}:=d v_{\dot{q}}^{-\alpha}+\Omega^{(0)} v_{\dot{q}}^{-\alpha}+\frac{1}{4} \Omega^{I J} \tilde{\gamma}_{\dot{q} \dot{p}}^{I J} v_{\dot{p}}^{-\alpha}=-\frac{1}{2} \Omega^{=I} v_{q}^{+\alpha} \gamma_{q \dot{q}}^{I} \\
& D v_{\dot{q}}^{+\alpha}:=d v_{\dot{q}}^{+\alpha}-\Omega^{(0)} v_{\dot{q}}^{+\alpha}+\frac{1}{4} \Omega^{I J} v_{\dot{p}}^{+\alpha} \gamma_{\dot{p} \dot{q}}^{I J}=-\frac{1}{2} \Omega^{\# I} v_{p}^{-\alpha} \gamma_{p \dot{q}}^{I}
\end{aligned}
$$

Notice that eqs. (5.22) can be used to find the derivatives or variations of the spinor helicity variable (4.8), while (5.24) gives the derivative/admissible variation of the polarization spinors (4.12).

The above equations have been written for $\mathrm{D}=10$ Lorentz harmonics, while the corresponding $D=11$ relation can be reproduced by identifying dotted and undotted indices in (5.22)-(5.25) and assuming that $I, J=1, \ldots, 9, \quad p, q=1, \ldots, 16$. In particular the $\mathrm{SO}(8)$ Klebsh-Gordan coefficients $\gamma_{p \dot{q}}^{I}$ in $11 \mathrm{D}$ case are replaced by $16 \times 16$ nine dimensional gamma matrices $\gamma_{p q}^{I}=\gamma_{q p}^{I}$. 


\subsection{Massless superparticle action in the analytical basis of Lorentz harmonic superspace}

Eqs. (5.22) allows us to specify the last term in $\mathrm{D}=10$ and $\mathrm{D}=11$ superstring action (5.1): after simple algebra using (3.26)-(3.30), we arrive at

$$
S=\int d \tau \mathcal{L}=\int d \tau \rho^{\#}(\tau)\left(D_{\tau} X^{=}-2 i D_{\tau} \theta_{q}^{-} \theta_{q}^{-}-X^{I} \Omega_{\tau}^{=I}\right)
$$

Here $\Omega_{\tau}^{=I}$ is the pull-back of the Cartan form $\Omega^{=I}=u^{=a} d u_{a}^{I}=-u_{a}^{I} d u^{=a}$ (see (5.15) and $(5.17))$ to the worldline divided by $d \tau$, generically

$$
\Omega^{=I}=d \tau \Omega_{\tau}^{=I}, \quad \Omega^{(0)}=d \tau \Omega_{\tau}^{(0)}, \quad \Omega^{I J}=d \tau \Omega_{\tau}^{I J},
$$

and

$$
D_{\tau} X^{=}=\partial_{\tau} X^{=}+2 \Omega_{\tau}^{(0)} X^{=}, \quad D_{\tau} \theta_{q}^{-}=\partial_{\tau} \theta_{q}^{-}+\Omega_{\tau}^{(0)} \theta_{q}^{-}+\frac{1}{4} \Omega_{\tau}^{I J} \theta_{p}^{-} \gamma_{p q}^{I J}
$$

(cf. (5.15) and (5.22)).

By construction, the action (5.26) is invariant under the rigid supersymmetry (5.9), $(5.10)$

$$
\delta_{\varepsilon} X^{=}=2 i \theta_{q}^{-} \varepsilon^{-q}, \quad \delta_{\varepsilon} X^{I}=2 i \theta_{q}^{-} \gamma_{q \dot{q}}^{I} \varepsilon^{+\dot{q}}, \quad \delta_{\varepsilon} \theta_{q}^{-}=\varepsilon^{-q}, \quad \delta_{\varepsilon} \Omega_{\tau}^{=I}=0, \quad \delta_{\varepsilon} \rho^{\#}=0 .
$$

As far as the $\kappa$-symmetry (3.32) is concerned, it is easy to check that all the fields in the action (5.26) are just invariant under it,

$$
\delta_{\kappa} X^{=}=0, \quad \delta_{\kappa} X^{I}=0, \quad \delta_{\kappa} \theta_{q}^{-}=0, \quad \delta_{\kappa} \Omega_{\bar{z}}^{=I}=0, \quad \delta_{\kappa} \rho^{\#}=0 .
$$

In this sense the $\kappa$-invariance of the analytic basis form of the massless superparticle action is trivial. Let us recall that this property is also characteristic for the pure twistor form of the Ferber-Schirafuji action, (2.34), the quantization of which results in the on-shell superfields of maximal 4D SYM and SUGRA theories.

\subsection{Massless $\mathrm{D}=10$ and $\mathrm{D}=11$ superparticle quantization in the analytical basis}

The quantization of massless 11D superparticle in analytical basis of Lorentz harmonic superspace has been considered in [38] and the 10D case can be carried out in a similar manner (see also $[78,79]$ where only vector harmonics were used). So we refer to [38] for the discussion on the structure of bosonic constraints and their resolution in classical and quantum theory, and concentrate here on the quantization of the fermionic variables (omitting the details when these can be found in [38]). Notice only that the worldline field $\rho^{\#}(\tau)$ becomes identified with the momentum $P=$ of the $X^{=}$coordinate function, $\rho^{\#}-P=\approx 0$, so that after quantization it can be replaced by $-i \hbar \partial_{=}=-i \hbar \frac{\partial}{\partial x^{=}}$(below, for shortness, we set $\hbar=1$ ). The fermionic constraints which follow from the action (5.26),

$$
d_{q}^{+}=-\pi_{q}^{+}+2 i \rho^{\#} \theta_{q}^{-} \approx 0, \quad \pi_{q}^{+}=-\frac{\partial L}{\partial \dot{\theta}_{q}^{-}},
$$


obey the Poisson bracket relations $\left(\left\{\theta_{q}^{-}, \pi_{p}^{+}\right\}_{P . B .}=\delta_{q p}\right)$

$$
\left\{d_{q}^{+}, d_{p}^{+}\right\}_{P . B .}=-4 i \rho^{\#} \delta_{q p}
$$

and hence are second class constraints in Dirac's classification [87].

After the quantization, as a consequences of the bosonic first class constraints and the (explicitly resolved) bosonic second class constraints, the 'wavefunction' of the superparticle depends on one bosonic coordinate $x^{=}$(or on its 'momentum' $\rho^{\#}$ ), on a set of homogeneous coordinates of $\mathbb{S}^{D-2}$ given by Lorentz harmonics $v_{\alpha q}^{-}$(see (4.14)), and on a set of fermionic variables. The type of these latter depends on the way of quantization chosen for the fermionic coordinate function $\theta_{q}^{-}$obeying the second class constraints (5.31) with characteristic Poisson brackets (5.32).

There is no $\mathrm{SO}(8)(\mathrm{SO}(9))$ covariant way to solve the second class constraints (5.31) explicitly. If one solves them implicitly by passing to Dirac brackets [87], one finds that $\theta_{q}^{-}$obey

$$
\left\{\theta_{q}^{-}, \theta_{p}^{-}\right\}_{D . B .}=-\frac{i}{4 \rho^{\#}} \delta_{q p}
$$

This implies that after quantization

$$
\hat{\theta}_{q}^{-}=\frac{1}{\sqrt{2 \rho^{\#}}} \mathfrak{C}_{q}
$$

where $\mathfrak{C}_{q}$ obey the Clifford algebra

$$
\left\{\mathfrak{C}_{q}, \mathfrak{C}_{p}\right\}=2 \delta_{q p} I, \quad\left\{\begin{array}{l}
q=1, \ldots, 8 \text { for } D=10 \\
q=1, \ldots, 16 \text { for } D=11 .
\end{array}\right.
$$

One might want to try a possibility to consider the wavefunction of the superparticle to be a Clifford superfield $\Phi\left(x^{=}, v_{\alpha q}^{-}, \mathfrak{C}_{q}\right)$. Similar approach to $\mathrm{D}=10$ superamplitudes, which implies their dependence on $n$ sets of Clifford variables $\mathfrak{C}_{q(i)}$, was developed in [25] (we briefly describe it in appendix A.2). However, as unconstrained $\Phi\left(x^{=}, v_{\alpha q}^{-}, \mathfrak{C}_{q}\right)$ contains the terms up to 8-th degree in $\mathfrak{C}_{q}$ in $\mathrm{D}=10$ and up to 16 -th degree in the case of $\mathrm{D}=11$, the Clifford superfield approach does not look economic and the corresponding description of amplitudes seems to be reducible (see also discussion in the Conclusion of recent [53]). A more economic constrained superfield description originates in an alternative quantization of the fermionic degrees of freedom of the $D=10$ and $D=11$ massless superparticle which we are going to describe now.

The idea of this alternative quantization can be followed back to the first studies of spinning particle mechanics $[88,89]$ in which the fermionic superpartners $\psi^{\mu}(\tau)$ of the bosonic coordinate function $x^{\mu}(\tau)$ obeys the Dirac brackets $\left\{\psi^{\mu}, \psi^{\nu}\right\}_{D . B .}=-2 i \eta^{\mu \nu}$. After quantization these produce the Clifford algebra commutation relation $\left\{\hat{\psi}^{\mu}, \hat{\psi}^{\nu}\right\}=2 \eta^{\mu \nu}$ for the fermionic operator. Then the standard way to arrive at Dirac (or Weyl) equation for the wavefunction of the spinning particle passes through using the representation of the fermionic operators by Dirac matrices, $\hat{\psi}^{\mu} \mapsto \Gamma^{\mu}{ }_{\alpha}^{\beta}$, and allowing the wavefunction to carry the corresponding spinorial index. 
Similar method was used in [90] in light-cone quantization of $\mathrm{D}=11$ superparticle and in [38] for covariant quantization and the study of hidden symmetries of this. There we identified the quantum version of the fermionic second class constraints with Clifford algebra valued element $\mathfrak{C}_{q}$,

$$
\hat{d}_{q}^{+}=\sqrt{2 \rho^{\#}} \mathfrak{C}_{q}
$$

and represented $\mathfrak{C}_{q}$ with $q=1, \ldots, 16$ by sixteen $256 \times 256$ gamma matrices acting on the 'wavefunction' of the 11D superparticle which belongs to 256 component Majorana spinor of $\mathrm{SO}(16)$ group. This is split on two 128 component Mayorana-Weyl spinors, one describing the on-shell fermionic gravitino degrees of freedom $\Psi_{I q}$ and other $(128=84+44)$ describing the bosonic on-shell degrees of freedom. Under $\mathrm{SO}(9)$ subgroup of $\mathrm{SO}(16)$ this latter can be decomposed on antisymmetric tensor $A_{I J K}(\mathbf{8 4})$ and symmetric traceless $h_{I J}$ (44). Choosing the block-anti-diagonal representation of the $\mathrm{d}=16$ gamma matrices, one finds that the action of the Gamma matrix on Majorana spinor mixes the two Majorana Weyl spinors,

$$
\begin{aligned}
\mathfrak{C}_{q} A_{I J K} & =3 \gamma_{q p[I J} \Psi_{K] p}, \quad \mathfrak{C}_{q} h_{I J}=2 \gamma_{q p(I} \Psi_{J) p}, \\
\mathfrak{C}_{q} \Psi_{I p} & =\gamma_{q p}^{J} h_{J I}+\frac{1}{3 !}\left(\gamma_{q p}^{I J K L}+6 \delta^{I[J} \gamma_{q p}^{K L]}\right) A_{J K L} .
\end{aligned}
$$

One can perform a similar quantization of the $\mathrm{D}=10$ superparticle. In it $\mathfrak{C}_{q}$ with $q=1, \ldots, 8$ are represented by eight $16 \times 16$ gamma matrices of $\mathrm{SO}(8)$ and the wavefunction is a 16 component Majorana spinor of $\mathrm{SO}(8)$ which can be split on two 8-dimensional representations. The triality of the Spin(8) group implies the equivalence of its two spinor and one vector representations, $8 \mathrm{~s}, 8 \mathrm{c}$ and $8 \mathrm{v}$, so that we can decompose the Majorana spinor on, say, $8 \mathrm{c}$ and $8 \mathrm{v}$ and identify these as a fermionic c-spinor $\psi_{\dot{q}}$ and a bosonic vector $w^{I}$. The action of the Majorana representation of gamma matrices on the Majorana spinor wavefunction is then described by (4.17) (in which the $\rho^{\#}$ multiplier can be removed by a proper redefinition of the fields),

$$
\mathfrak{C}_{q} \psi_{\dot{q}}=i \gamma_{q \dot{q}}^{I} w^{I}, \quad \mathfrak{C}_{q} w^{I}=\gamma_{q \dot{q}}^{I} \psi_{\dot{q}}
$$

Superfields collecting the above supermultiplets of fields will be the on-shell superfields of $D=11$ SUGRA and $D=10$ SYM supermultiplets, the counterpart of $D=4$ superfield (2.24). To obtain such superfields in superparticle quantization, we use a new (to the best of our knowledge) manner of quantization of the system with second class constraints, in which the state vector is represented by multi-component field(s) and the quantum second class constraints act on it as differential operators mixing the components of the state vector rather than annihilating them.

Namely, when quantizing the dynamical system described by the action (5.26), instead of passing to Dirac brackets (5.33), we allow the quantum counterparts $\hat{d}_{q}^{+}$of the second class constraints $d_{q}^{+}$to obey the Clifford-like algebra (as follows from straightforward quantization of (5.32)), realize them as differential operators in an appropriate on-shell superspace, $\hat{d}_{q}^{+}=D_{q}^{+}$, introduce an appropriate set of superfields in this on-shell superspace (these can be guessed from (5.38) and (5.37) for $\mathrm{D}=10$ and $\mathrm{D}=11$ ) and assume that 
the differential operator $D_{q}^{+}$acts on this set of superfields in the way which is essentially determined by (5.38) and (5.37).

The result of such a quantization can be called constrained on-shell superfield formalism. Interestingly enough, such on-shell superfield formalism had been proposed in [35] without any discussion of a superparticle model.

\subsubsection{On-shell superspaces for 10D SYM and 11D SUGRA}

The $\mathrm{D}=10$ and $\mathrm{D}=11$ on-shell superfields are defined on the superspace with bosonic coordinates $x^{=}$and $v_{\alpha q}^{-}$, and fermionic $\theta_{q}^{-}$coordinates,

$$
\begin{array}{rlrl}
\Sigma^{\left(D-1 \mid n_{D}\right)}: & \left\{\left(x^{=}, \theta_{q}^{-}, v_{\alpha q}^{-}\right)\right\}, & \left\{v_{\alpha q}^{-}\right\}=\mathbb{S}^{D-2}, \\
& q=1, \ldots, n_{D}, \quad \alpha=1, \ldots, 2 n_{D}, \quad n_{D}=\left\{\begin{array}{cc}
8 & \text { for } D=10 \\
16 & \text { for } D=11 .
\end{array}\right.
\end{array}
$$

which, hence, are called $\mathrm{D}=10$ and $\mathrm{D}=11$ on-shell superspaces. The generic superfields on these superspaces contain too many component fields so that $\mathrm{D}=10$ and $\mathrm{D}=11$ on-shell superfields on $\Sigma^{\left(D-1 \mid n_{D}\right)}$ cannot be unconstrained, i.e. they should obey some superfield equations.

To arrive at these equations when quantizing 10D and 11D massless superparticle, we begin by realizing the quantum version of the constraint $\hat{d}_{q}^{+}$as differential operator on $(5.39)$

$$
D_{q}^{+}=\partial_{q}^{+}+2 i \theta_{q}^{-} \partial_{=}, \quad \partial_{=}:=\frac{\partial}{\partial x^{=}}, \quad \partial_{q}^{+}:=\frac{\partial}{\partial \theta_{q}^{-}} .
$$

Then, as the fermionic constraint is of the second class, i.e. the anticommutator of two such constraints is nonzero,

$$
\left\{D_{q}^{+}, D_{p}^{+}\right\}=4 i \delta_{q p} \partial_{=},
$$

we cannot assume that the action of $D_{q}^{+}$annihilates the state vector, i.e. we should expect the presence of a nonvanishing r.h.s. in the equation reflecting the existence of the second class constraint by defining the action of $D_{q}^{+}$on the state vector (wavefunction). ${ }^{15}$ This can be done consistently if the wavefunction carries certain indices of $\mathrm{SO}(D-2)$ group.

To find the index structure of the wavefunctions and the r.h.s.-s of the above mentioned equations, it is convenient to notice that (5.41) differs from the Clifford algebra by the presence of $\partial_{=}$in the r.h.s. Then the superfield equations which should be imposed on the on-shell superfield to reduce their field content to the fields describing 10D SYM and 11D supergravity multiplets are suggested by the action of the formal Clifford algebra elements on the fields of these multiplets: (5.38) and (5.37).

\footnotetext{
${ }^{15} \mathrm{An}$ analytic superfield formalism of [53] is based on homogeneous equations, but requires breaking of $\mathrm{SO}(D-2)$ symmetry down to $\mathrm{SO}(D-4)$ by splitting $D_{q}^{+}$on two sets of complex operators $D^{A}$ and $\bar{D}_{A}$ with $A=1,2,3,4$.
} 


\subsubsection{On-shell superfields and superfield equations of 10D SYM}

The basic $\mathrm{D}=10$ equations [35] are imposed on the fermionic superfield $\Psi_{\dot{q}}=\Psi_{\dot{q}}\left(x^{=}, \theta_{\dot{q}}^{-}, v_{\alpha \dot{q}}{ }^{-}\right)$ carrying $c$-spinor index of $\mathrm{SO}(8)$. They read

$$
D=10: \quad D_{q}^{+} \Psi_{\dot{q}}=\gamma_{q \dot{q}}^{I} \partial_{=} W^{I}, \quad q=1, \ldots, 8, \quad \dot{q}=1, \ldots, 8, \quad I=1, \ldots, 8 .
$$

The superfield $W^{I}$ is defined by eq. (5.42) itself which also implies that it obeys ${ }^{16}$

$$
D_{q}^{+} W^{I}=2 i \gamma_{q \dot{q}}^{I} \Psi_{\dot{q}}
$$

This equation shows that there are no other independent components in the constrained on-shell superfield $\Psi_{\dot{q}}$. Indeed, one can find that its decomposition in the powers of $\theta_{\dot{q}}^{-}$reads

$$
\begin{aligned}
& \Psi_{\dot{q}}\left(x^{=}, v_{q}^{-} ; \theta_{q}^{-}\right)=\psi_{\dot{q}}\left(x^{=}, v_{q}^{-}\right)+\theta_{q}^{-} \gamma_{\dot{q}}^{I} \partial_{=} w^{I}\left(x^{=}, v_{q}^{-}\right)+ \\
& +\sum_{k=1}^{4}(-i)^{k} \frac{(2 k-1) ! !}{(2 k) ! !(2 k) !}\left(\theta^{-} \gamma^{I_{k-1} I_{k}} \theta^{-}\right) \ldots\left(\theta^{-} \gamma^{I_{1} I_{2}} \theta^{-}\right)\left(\gamma^{I_{1} I_{2}} \ldots \gamma^{I_{k-1} I_{k}}\right)_{\dot{q} \dot{p}}\left(\partial_{=}\right)^{k} \psi_{\dot{p}}+ \\
& +\sum_{k=1}^{3}(-i)^{k} \frac{(2 k) ! !}{(2 k+1) ! !(2 k+1) !}\left(\theta^{-} \gamma^{I_{1} I_{2}} \theta^{-}\right) \ldots\left(\theta^{-} \gamma^{I_{k-1} I_{k}} \theta^{-}\right)\left(\tilde{\gamma}^{I_{1} I_{2}} \ldots \tilde{\gamma}^{I_{k-1} I_{k}} \tilde{\gamma}^{I} \theta^{-}\right)_{\dot{q}}\left(\partial_{=}\right)^{k+1} w^{I} .
\end{aligned}
$$

Hence, as it was stated already in [35], this constrained superfield describes on-shell degrees of freedom of SYM multiplet.

\subsubsection{On-shell SUSY and supersymmetric invariant of the (linearized) 10D SYM}

The supersymmetry transformations of the leading components of bosonic and fermionic on-shell superfields

$$
\left.\Psi_{\dot{q}}\right|_{0}=\psi_{\dot{q}},\left.\quad W^{I}\right|_{0}=w^{I} .
$$

can be extracted from the superfield equations (5.42) and (5.43). They have the form already announced in (4.17),

$$
\delta_{\epsilon} \psi_{\dot{q}}=\epsilon^{-q} \gamma_{q \dot{q}}^{I} \partial_{=} w^{I}, \quad \delta_{\epsilon} w^{I}=2 i \epsilon^{-q} \gamma_{q \dot{q}}^{I} \psi_{\dot{q}} .
$$

It is also not difficult to find the following supersymmetric invariant

$$
I_{10 D}=\int d x^{=}\left(\partial_{=} w^{I} \partial_{=} w^{I}+2 i \partial_{=} \psi_{\dot{q}} \psi_{\dot{q}}\right),
$$

and to observe that it can be written in terms of superfields as follows

$$
I_{10 D}=\left.\frac{1}{16} \int d x^{=} D_{q}^{+}\left(\Psi_{\dot{q}} \overleftrightarrow{D_{q}^{+}} \Psi_{\dot{q}}\right)\right|_{0}
$$

\footnotetext{
${ }^{16}$ To be more precise, eq. (5.42) determines the form of $V^{I}=\partial_{=} W^{I}$. Just this superfield was used in [35].
} 
Eq. (5.48) can be considered as an integral over a $(1,8)$ dimensional subsuperspace $\left\{\left(x^{=}, \theta_{q}^{-}\right)\right\}=\mathfrak{L}^{(1 \mid 8)}$ of $10 \mathrm{D}$ on-shell superspace $\Sigma^{(9 \mid 8)}$ but taken with an exotic superspace measure similar to one used in $[92,93]$ to construct a worldsheet superfield formulation of the heterotic string (a formal discussion on integral forms in superspace and applications to $3 \mathrm{~d}$ superfield theories can be found in [94]). Notice also that our supersymmetric invariants depend on the spinor frame variables $v_{\alpha q}^{-}$which is possible as these are inert under supersymmetry.

For our discussion below it will be important that one can also write similar supersymmetric invariant containing two on-shell superfields, $\Psi_{\dot{q}}\left(x^{=}, v_{\alpha q}^{-}, \theta_{q}^{-}\right)$and $\tilde{\Psi}_{\dot{q}}\left(x^{=}, v_{\alpha q}^{-}, \theta_{q}^{-}\right)$. Indeed, one can check that

$$
\begin{aligned}
\mathcal{I}_{10 D}=\left.\frac{1}{16} \int d x^{=} D_{q}^{+}\left(\widetilde{\Psi}_{\dot{q}} \overleftrightarrow{D_{q}^{+}} \Psi_{\dot{q}}\right)\right|_{0} & \left.\equiv \frac{1}{16} \int d x^{=} D_{q}^{+}\left(\widetilde{\Psi}_{\dot{q}} D_{q}^{+} \Psi_{\dot{q}}+D_{q}^{+} \widetilde{\Psi}_{\dot{q}} \Psi_{\dot{q}}\right)\right|_{0}= \\
& =\int d x=\left(\partial_{=} \widetilde{w}^{I} \partial_{=} w^{I}+i \partial_{=} \widetilde{\psi}_{\dot{q}} \psi_{\dot{q}}-i \widetilde{\psi}_{\dot{q}} \partial_{=} \psi_{\dot{q}}\right)
\end{aligned}
$$

is invariant under supersymmetry defined by (5.46) and

$$
\delta_{\epsilon} \widetilde{\psi}_{\dot{q}}=\epsilon^{-q} \gamma_{q \dot{q}}^{I} \partial_{=} \widetilde{w}^{I}, \quad \delta_{\epsilon} \widetilde{w}^{I}=2 i \epsilon^{-q} \gamma_{q \dot{q}}^{I} \widetilde{\psi}_{\dot{q}}
$$

Even more useful for studying superamplitudes will be the expression of the above invariant in terms of Fourier images of fields and superfields

$$
\begin{aligned}
\widetilde{\Psi}_{\dot{q}}\left(\rho^{\#}, v^{-}, \theta^{-}\right): & =\frac{1}{\sqrt{2 \pi}} \int d x^{=} e^{-i x^{=} \rho^{\#}} \widetilde{\Psi}_{\dot{q}}\left(x^{=}, v^{-}, \theta^{-}\right)= \\
& =\widetilde{\psi}_{\dot{q}}\left(\rho^{\#}, v^{-}\right)+i \rho^{\#} \theta_{q}^{-} \gamma_{q \dot{q}}^{I} \widetilde{w}^{I}\left(\rho^{\#}, v^{-}\right)+\mathcal{O}\left(\theta_{q}^{-} \theta_{p}^{-}\right)
\end{aligned}
$$

The integrand of this expression reads

$$
\begin{aligned}
\Im_{10 D}\left(\rho^{\#}\right) & =\left.\left.\frac{1}{16} D_{q}^{+}\left(\widetilde{\Psi}_{\dot{q}}\left(\rho^{\#}\right) \overleftrightarrow{D_{q}^{+}} \Psi_{\dot{q}}\left(-\rho^{\#}\right)\right)\right|_{0} \equiv \frac{1}{16} D_{q}^{+}\left(\widetilde{\Psi}_{\dot{q}} D_{q}^{+} \Psi_{\dot{q}}+D_{q}^{+} \widetilde{\Psi}_{\dot{q}} \Psi_{\dot{q}}\right)\right|_{0}= \\
& =\widetilde{w}^{I}\left(\rho^{\#}\right)\left(\rho^{\#}\right)^{2} w^{I}\left(-\rho^{\#}\right)-2 \rho^{\#} \widetilde{\psi}_{\dot{q}}\left(\rho^{\#}\right) \psi_{\dot{q}}\left(-\rho^{\#}\right)
\end{aligned}
$$

where we have written only the argument essential for the discussion below, so that $\widetilde{\Psi}_{\dot{q}}\left(\rho^{\#}\right) \equiv \widetilde{\Psi}_{\dot{q}}\left(\rho^{\#}, v_{q}^{-}, \theta_{q}^{-}\right)$etc., and the covariant derivative has the form

$$
D_{q}^{+}=\partial_{q l}^{+}-2 \rho^{\#} \theta_{q}^{-}, \quad \partial_{q}^{+}:=\frac{\partial}{\partial \theta_{q}^{-}}
$$

To avoid confusion when reproducing (5.52), we notice that in the expression $D_{q}^{+} \widetilde{\Psi}_{\dot{q}}\left(\rho^{\#}\right)$ the derivative $D_{q}^{+}$does have the form (5.54), which can be denoted by $D_{q}^{+}\left(\rho^{\#}\right)$, while in $D_{q}^{+} \Psi_{\dot{q}}\left(-\rho^{\#}\right)$ we should use $D_{q}^{+}\left(-\rho^{\#}\right)=\partial_{q_{l}}^{+}+2 \rho^{\#} \theta_{q}^{-}$. This reflects the fact that the action of the fermionic covariant derivative is actually defined in $x^{=}$-coordinate representation, and, its momentum, $\rho^{\#}$-representation (5.54) is restored from that. 


\subsubsection{On-shell superfields, superfield equations and supersymmetric invariant of linearized 11D SUGRA}

Similarly, observing (5.37) one can guess that the linearized 11D supergravity is described by a bosonic antisymmetric tensor superfield $\Phi^{I J K}=\Phi^{[I J K]}\left(x^{=}, \theta_{q}^{-}, v_{\alpha q}^{-}\right)$which obeys the superfield equation [35]

$$
D=11: \quad D_{q}^{+} \Phi^{I J K}=3 i \gamma_{q p}^{[I J} \Psi_{p}^{K]}, \quad \gamma_{q p}^{I} \Psi_{p}^{I}=0, \quad q, p=1, \ldots, 16, \quad I=1, \ldots, 8,9 .
$$

The consistency of eq. (5.55) requires

$$
\begin{aligned}
D=11: \quad D_{q}^{+} \Psi_{p}^{I} & =\frac{1}{18}\left(\gamma_{q p}^{I J K L}+6 \delta^{I[J} \gamma_{q p}^{K L]}\right) \partial_{=} \Phi^{J K L}+2 \partial_{=} H_{I J} \gamma_{q p}^{J}, \\
D_{q}^{+} H_{I J} & =i \gamma_{q p}^{(I} \Psi_{p}^{J)}, \quad H_{I J}=H_{J I}, \quad H_{I I}=0 .
\end{aligned}
$$

These equations can be used to extract the supersymmetry transformations of the leading components of superfields

$$
D=11: \quad \phi_{I J K}:=\phi_{I J K}\left(x^{=}, v_{q}^{-}\right)=\left.\Phi_{I J K}\right|_{\theta_{q}^{-}=0}, \quad \psi_{p}^{I}=\left.\Psi_{p}^{I}\right|_{\theta_{q}^{-}=0}, \quad h_{I J}=\left.H_{I J}\right|_{\theta_{q}^{-}=0} .
$$

These are

$$
\begin{aligned}
\delta_{\epsilon} \phi_{I J K} & =3 i \epsilon^{-} \gamma_{[I J} \psi_{K]}, \quad \delta_{\epsilon} h_{I J}=i \epsilon^{-} \gamma_{(I} \psi_{J)}, \\
\delta_{\epsilon} \psi_{q}^{I} & =\frac{1}{18}\left(\epsilon^{-} \gamma^{I J K L}+6 \delta^{I J} \epsilon^{-} \gamma^{K L]}\right)_{q} \partial_{=} \phi^{J K L}+2 \partial_{=} h_{I J}\left(\epsilon^{-} \gamma^{J}\right)_{q} .
\end{aligned}
$$

One can easily find that the following linearized action is invariant (modulo integral of total derivative) under the rigid supersymmetry (5.59):

$$
I_{11 D}=\int d x=\left(2 \partial_{=} h_{I J} \partial_{=} h_{I J}+\frac{1}{6} \partial_{=} \phi_{I J K} \partial_{=} \phi_{I J K}+i \partial_{=} \psi_{I q} \psi_{I q}\right) .
$$

One can also check that the integrand of (5.60) can be identified with the leading component of the composed on-shell superfield $D_{q}^{+}\left(\Psi_{I p} D_{q}^{+} \Psi_{I p}\right)$,

$$
\begin{aligned}
\left.\frac{1}{32} D_{q}^{+}\left(\Psi_{I p} D_{q}^{+} \Psi_{I p}\right)\right|_{0} & =2 \partial_{=} h_{I J} \partial_{=} h_{I J}+\frac{1}{6} \partial_{=} \phi_{I J K} \partial_{=} \phi_{I J K}+i \partial_{=} \psi_{I q} \psi_{I q}, \\
q, p & =1, \ldots, 16, \quad I, J, K=1, \ldots, 9,
\end{aligned}
$$

so that our invariant can be written in the form

$$
I_{11 D}=\left.\frac{1}{32} \int d x^{=} D_{q}^{+}\left(\Psi_{I p} D_{q}^{+} \Psi_{I p}\right)\right|_{0} .
$$

This can be considered as an integral over a superline $\left.\mathfrak{L}^{(1 \mid 16)}=\left(x^{=}, \theta_{q}^{-}\right)\right\}$in the 11D onshell superspace $\Sigma^{(10 \mid 16)}$ but taken with an exotic superspace measure (see comments on the 10D case, eq. (5.48) and below it). Clearly, the value of the integral over the superline depend on the spinor frame variables $v_{\alpha q}^{-}$, which are inert under supersymmetry (5.12). 
Like in the 10D SYM case, we can also write the invariant including two different supergravity superfields

$$
\begin{aligned}
\mathcal{I}_{11 D} & =\left.\frac{1}{32} \int d x^{=} D_{q}^{+}\left(\widetilde{\Psi}_{I p} \overleftrightarrow{D}_{q}^{+} \Psi_{I p}\right)\right|_{0}= \\
& =\int d x=\left(4 \partial_{=} \widetilde{h}_{I J} \partial_{=} h_{I J}+\frac{1}{3} \partial_{=} \widetilde{\phi}_{I J K} \partial_{=} \phi_{I J K}+i \partial_{=} \widetilde{\psi}_{I q} \psi_{I q}-i \widetilde{\psi}_{I q} \partial_{=} \psi_{I q}\right) \\
q, p & =1, \ldots, 16, \quad I, J, K=1, \ldots, 9
\end{aligned}
$$

Below we will generalize the above on-shell superfield representation for the case of three amplitudes of 10D SYM and linearized 11D supergravity and present a candidate for generalization of the BCFW recurrent relations [16] for such superamplitudes. The equations for supersymmetric invariants, (5.49) and (5.63), will be useful when writing these candidate BCFW-type relations for 10D and 11D superamplitudes [30].

\section{Superfield representation for 10D SYM and 11D SUGRA amplitudes}

In this section we first introduce the tree superamplitudes of 10D SYM and 11D SUGRA as multiparticle counterparts of the corresponding on-shell superfields (sections 6.1 and 6.2). These latter suggest the index structure of the amplitudes, the variables they depend on, as well as the set of differential equations these obey.

As we have already stressed at the beginning of section 4, we are working with the on-shell superamplitudes so that we do not use neither Lagrangian nor Feynman rules in their derivation. The experience gained in $\mathrm{D}=4$ suggests to search for a BCFW-type recurrent relations to calculate the on-shell superamplitudes starting from the basic 3-point superamplitudes. Those include on-shell superamplitudes dependent on deformed helicity spinors and fermionic variables. In section 6.3 we generalize the BCFW deformations for the case of 10D SYM and 11D SUGRA superamplitudes. The candidate BCFW recurrent relations are discussed in sections 6.4-6.6. In this latter part the results are preliminary.

\subsection{Superfield representation of the 10D SYM amplitudes: 10D superamplitudes}

The structure of the constrained on-shell superfields of 10D SYM and of the equations imposed on them, (5.42) and (5.43), suggests to define a set of bosonic superfield amplitudes or bosonic superamplitudes

$$
\mathcal{A}_{I_{1} \ldots I_{l} \ldots I_{n}}^{(n)}\left(\left\{\rho_{(i)}^{\#}\right\} ;\left\{v_{q(i)}^{-}\right\} ;\left\{\theta_{q(i)}^{-}\right\}\right)=: \mathcal{A}_{\ldots I_{l} \ldots}^{(n)}\left(\ldots ; k_{l}, \theta_{l}^{-} ; \ldots\right)
$$

depending, in addition to spinor helicity variables $v_{\alpha q(i)}^{-}$and 'energies' $\rho_{(i)}^{\#}$, on $n$-sets of fermionic $8 \mathrm{~s}-$ spinor variables $\theta_{q(i)}^{-}$, and carrying a $8 \mathrm{v}$ index for each of the scattered particles. The r.h.s. of (6.1) shows the schematic notation which we will use below when it cannot result in a confusion. The leading component $\left(\theta_{q(i)}^{-}=0\right.$ 'value') of this superfield amplitude is a particular case of the 'component' amplitude in (4.24), the purely bosonic amplitude $\mathcal{A}_{\ldots I_{l} \ldots}^{(n)}\left(\left\{\rho_{(i)}^{\#}\right\} ;\left\{v_{q(i)}^{-}\right\}\right)$, where multidotes also denote the $8 \mathrm{v}$ indices. 
The superamplitudes (6.1) obey the following equations

$$
D_{q_{l}}^{+(l)} \mathcal{A}_{\ldots I_{l} \ldots}^{(n)}\left(\ldots ; k_{l}, \theta_{l}^{-} ; \ldots\right)=2 i \gamma_{q_{l} \dot{q}_{l}}^{I_{l}} \mathcal{A}_{\ldots \dot{q}_{l} \ldots}^{(n)}\left(\ldots ; k_{l}, \theta_{l}^{-} ; \ldots\right)
$$

which are suggested by the equations (5.43) for the on-shell superfields. In (6.2) the fermionic covariant derivatives have the form (cf. (5.54))

$$
D_{q_{l}}^{+(l)}=\partial_{q_{l}}^{+(l)}-2 \rho_{(l)}^{\#} \theta_{q(l)}^{-} \quad, \quad \partial_{q_{l}}^{+(l)}:=\frac{\partial}{\partial \theta_{q(l)}^{-}}
$$

and obey

$$
\left\{D_{q}^{+(l)}, D_{p}^{+(j)}\right\}=-4 \delta_{l l^{\prime}} \delta_{q p} \rho_{(l)}^{\#} .
$$

The fermionic superamplitudes in the r.h.s. of (6.2),

$$
\mathcal{A}_{\ldots \dot{q}_{l} \ldots}\left(\ldots ; k_{l}, \theta_{l}^{-} ; \ldots\right)=\mathcal{A}_{I_{1} \ldots I_{l-1} \dot{q}_{l} I_{l+1} \ldots I_{n}}^{(n)}\left(\left\{\rho_{(i)}^{\#}, v_{q(i)}^{-} ; \theta_{\dot{q}(i)}^{-}\right\}\right)
$$

are defined by contractions of eq. (6.2) with $\gamma_{q_{l} \dot{p}_{l}}^{I_{l}}$ and, by consistency of (6.2), obey

$$
D_{q_{l}}^{+(l)} \mathcal{A}_{\ldots \dot{q}_{l} \ldots}^{(n)}\left(\ldots ; k_{l}, \theta_{l}^{-} ; \ldots\right)=i(-)^{\Sigma_{l}} \gamma_{q_{l} \dot{q}_{l}}^{I_{l}} \rho_{l}^{\#} \mathcal{A}_{\ldots I_{l} \ldots}^{(n)}\left(\ldots ; k_{l}, \theta_{l}^{-} ; \ldots\right) .
$$

The sign factors $(-)^{\Sigma_{l}}$ are introduced here to make the equation applicable for a more general case, where some of the indices denoted by multi-dots are fermionic ( $c$-spinor ones). In the case under consideration $(-)^{\Sigma_{l}}=1$; see eq. (6.11) below for generic case. Eqs. (6.2) and (6.5) imply

$$
\mathcal{A}_{\ldots \dot{q}_{l} \ldots}^{(n)}\left(\ldots ; k_{l}, \theta_{l}^{-} ; \ldots\right)=\mathcal{A}_{\ldots \dot{q}_{l} \ldots}^{(n)}\left(\ldots ; k_{l} ; \ldots\right)+(-)^{\Sigma_{l}} \theta_{q l}^{-} \gamma_{q_{l} \dot{q}_{l}}^{I_{l}} \mathcal{A}_{\ldots I_{l} \ldots}^{(n)}\left(\ldots ; k_{l} ; \ldots\right)+\mathcal{O}\left(\theta_{l}^{-} \gamma^{J K} \theta_{l}^{-}\right)
$$

where $\mathcal{A}_{\ldots \dot{q}_{l} \ldots}^{(n)}\left(\ldots ; k_{l} ; \ldots\right)=\mathcal{A}_{\ldots \dot{q}_{l} \ldots}^{(n)}\left(\ldots ; k_{l}, 0 ; \ldots\right)$ and $\mathcal{A}_{\ldots I_{l} \ldots}^{(n)}\left(\ldots ; k_{l} ; \ldots\right)=\mathcal{A}_{\ldots I_{l} \ldots}^{(n)}\left(\ldots ; k_{l}, 0 ; \ldots\right)$ may depend on other fermionic coordinates, $\theta_{j}^{-}$with $j \neq l$. By analogy with decomposition of the on-shell superfield, we can show that the terms of higher order in $l$-th fermionic coordinate, $\mathcal{O}\left(\theta_{l}^{-} \gamma^{J K} \theta_{l}^{-}\right)$, are expressed through $\mathcal{A}_{\ldots q_{l} \ldots}^{(n)}\left(\ldots ; k_{l} ; \ldots\right), \mathcal{A}_{\ldots I_{l} \ldots}^{(n)}\left(\ldots ; k_{l} ; \ldots\right)$ and $\rho_{l}^{\#}$.

Using the algebra of covariant derivatives, one can extend the above line of arguments and to obtain the equations for the generic superamplitude,

$$
\mathcal{A}_{Q_{1} \ldots Q_{l} \ldots Q_{n}}^{(n)}\left(k_{1}, \theta_{1}^{-} ; \ldots ; k_{l}, \theta_{l}^{-} ; \ldots ; k_{n}, \theta_{n}^{-}\right)=: \mathcal{A}_{\ldots Q_{l} \ldots}^{(n)}\left(\ldots ; k_{l}, \theta_{l}^{-} ; \ldots\right),
$$

with a number of $8 \mathrm{v}$ and a number of $8 \mathrm{c}$ indices,

$$
Q_{l}=\left(I_{l}, \dot{q}_{l}\right), \quad I_{l}=1, \ldots, 8, \quad \dot{q}_{l}=1, \ldots, 8
$$

The superfield equations for the generic superamplitude read

$$
D_{q_{l}}^{+(l)} \mathcal{A}_{Q_{1} \ldots Q_{l} \ldots Q_{n}}^{(n)}\left(\ldots ; k_{l}, \theta_{l}^{-} ; \ldots\right)=(-)^{\Sigma_{l}} \Delta_{q_{l} Q_{l} P_{l}} \mathcal{A}_{Q_{1} \ldots P_{l} \ldots Q_{n}}^{(n)}\left(\ldots ; k_{l}, \theta_{l}^{-} ; \ldots\right),
$$


where (cf. (4.29))

$$
\Delta_{q Q P}:=i \gamma_{q \dot{q}}^{I}\left(2 \delta_{Q}^{I} \delta_{P}^{\dot{q}}+\rho^{\#} \delta_{Q}^{\dot{q}} \delta_{P}^{I}\right),
$$

and the integer $\Sigma_{l}$ counts (modulo 2) a number of the fermionic $\dot{q}_{j}$ indices among $Q_{j}$ 's with $j=1, \ldots,(l-1),{ }^{17}$

$$
D=10: \quad \Sigma_{l}=\sum_{j=1}^{l-1} \frac{\left(1-(-)^{\varepsilon\left(Q_{j}\right)}\right)}{2}, \quad \varepsilon\left(I_{j}\right)=0, \quad \varepsilon\left(\dot{q}_{j}\right)=1 .
$$

Eqs. (6.9) contain (6.5) and

$$
D_{q_{l}}^{+(l)} \mathcal{A}_{\ldots I_{l} \ldots}^{(n)}\left(\ldots ; k_{l}, \theta_{l}^{-} ; \ldots\right)=2 i(-)^{\Sigma_{l}} \gamma_{q_{l} \dot{q}_{l}}^{I_{l}} \mathcal{A}_{\ldots \dot{q}_{l} \ldots}^{(n)}\left(\ldots ; k_{l}, \theta_{l}^{-} ; \ldots\right) .
$$

(6.2) is a particular case of this equation in which all the indices denoted by multidots are bosonic (8v); obviously, it is also correct for the case when the set $Q_{1} \ldots Q_{(l-1)}$ contains an even number of $\dot{q}_{j}$ 's.

As in the case of (6.6), one can decompose the generic superamplitudes $\mathcal{A}_{Q_{1} \ldots Q_{l} \ldots Q_{n}}^{(n)}$ $\left(\ldots ; k_{l}, \theta_{l}^{-} ; \ldots\right)$ and define l-type-component amplitudes $\mathcal{A}_{Q_{1} \ldots Q_{l} \ldots Q_{n}}^{(n)}\left(\ldots ; k_{l} ; \ldots\right)=$ $\left.\mathcal{A}_{Q_{1} \ldots Q_{l} \ldots Q_{n}}^{(n)}\right|_{\theta_{l}^{-}=0}$ which depend on other fermionic variables $\theta_{q j}^{-}$with $j \neq l$. Their supersymmetry transformations are essentially described by eqs. (4.25): $l$-th projection of supersymmetry mixes them as in (4.25) while all the others are essentially given by supertranslations of fermionic coordinates $\theta_{q j}^{-}$with $j \neq l$. Then the combination (5.50) of such l-type-component amplitudes is invariant under l-th projection of supersymmetry and one can ask what is the superfield representation of that. This appears to coincide with leading $l$-component $\left(\theta_{l}=0\right.$ 'value') of

$$
D_{q_{l}}^{+(l)}\left(\mathcal{A}_{\ldots . \dot{q}_{l}}^{\left(n^{\prime}\right)}\left(\ldots ; k_{l}, \theta_{l}^{-}\right) \overleftrightarrow{D}_{q_{l}}^{+(l)} \mathcal{A}_{\dot{q}_{l} \ldots}^{(n)}\left(-k_{l}, \theta_{l}^{-} ; \ldots\right)\right)
$$

where $\mathcal{A}^{(l)} \overleftrightarrow{D}_{q_{l}}^{+(l)} \mathcal{A}^{(n)}=\mathcal{A}^{(l)} D_{q_{l}}^{+(l)} \mathcal{A}^{(n)}-(-)^{\varepsilon\left(\mathcal{A}^{\left(n^{\prime}\right)}\right)} D_{q_{l}}^{+(l)} \mathcal{A}^{\left(n^{\prime}\right)} \mathcal{A}^{(n)}$ and $\varepsilon\left(\mathcal{A}^{(l)}\right)$ denotes the Grassmann parity of the superamplitude $\mathcal{A}^{(l)}$.

This in its turn can be considered as a superspace integral with an exotic superspace measure (cf. (5.48) and see comment below this equation)

$$
\left.\int d x_{l}^{=}(-)^{\Sigma_{l}} D_{q_{l}}^{+(l)}\left(\mathcal{A}_{\ldots \dot{q}_{l}}^{(l)}\left(\ldots ; x_{l}^{=}, v_{p l}^{-}, \theta_{p l}^{-}\right) \overleftrightarrow{D_{q_{l}}^{+(l)}} \mathcal{A}_{\ldots \dot{q}_{l} \ldots}^{(n)}\left(x_{l}^{=}, v_{p l}^{-}, \theta_{p l}^{-} ; \ldots\right)\right)\right|_{\theta_{q l}^{-}=0}
$$

of the fourier image of the superamplitudes with respect to $\rho_{l}^{\#}$,

$$
\mathcal{A}_{\ldots \dot{q} l \ldots}^{(n)}\left(\ldots ; x_{l}^{=}, v_{p l}^{-}, \theta_{p l}^{-} ; \ldots\right)=\int d \rho_{l}^{\#} e^{i x_{l}} \rho_{l}^{\#} \mathcal{A}_{\ldots \dot{q} l \ldots}^{(n)}\left(\ldots ; \rho_{l}^{\#}, v_{p l}^{-}, \theta_{p l}^{-} ; \ldots\right)
$$

Notice that in (6.14) $D_{q_{l}}^{+(l)}$ is the derivative in the coordinate representation,

$$
D_{q_{l}}^{+(l)}=\frac{\partial}{\partial \theta_{q l}^{-}}+2 i \theta_{q l}^{-} \frac{\partial}{\partial x_{l}^{=}} \equiv \partial_{q l}^{+}+2 i \theta_{q l}^{-} \partial_{=}^{l} .
$$

\footnotetext{
${ }^{17}$ The choice of definition of $\Sigma_{l}$ is not unique. It corresponds to a prescription of the ordering of $\theta_{q_{i} i}^{-}$ in the decomposition of the superfield amplitudes. Such type ambiguity is similar to the one appearing in defining the old-fashioned form of the operator approach to the second quantization of non-relativistic fermionic field which can be found e.g. in [91].
} 


\subsection{Tree superamplitudes in $\mathrm{D}=11$}

The on-shell superfield description of linearized 11D supergravity, as described by eqs. (5.55), (5.56) and (5.57), suggests to consider the superfield amplitudes carrying a set of three antisymmetrized indices of $\mathrm{SO}(9)$ group,

$$
\mathcal{A}_{\left[I_{1} J_{1} K_{1}\right] \ldots\left[I_{l} J_{l} K_{l}\right] \ldots\left[I_{n} J_{n} K_{n}\right]}^{(n)}\left(\left\{\rho_{i}^{\#}\right\} ;\left\{v_{q i}^{-}\right\} ;\left\{\theta_{q i}^{-}\right\}\right)=: \mathcal{A}_{\ldots\left[I_{l} J_{l} K_{l}\right] \ldots}^{(n)}\left(\ldots ; k_{l}, \theta_{l}^{-} ; \ldots\right) .
$$

Such a bosonic superamplitude obeys $n$ superfield equations

$$
D_{q_{l}}^{+(l)} \mathcal{A}_{\ldots\left[I_{l} J_{l} K_{l}\right] \ldots}^{(n)}\left(\ldots ; k_{l}, \theta_{l}^{-} ; \ldots\right)=(-)^{\Sigma_{l}} 3 i \gamma_{\left[J_{l} K_{l} \mid q_{l} p_{l}\right.} \mathcal{A}_{\left.\ldots \mid I_{l}\right] p_{l} \ldots}^{(n)}\left(\ldots ; k_{l}, \theta_{l}^{-} ; \ldots\right), \quad l=1, \ldots, n
$$

where $D_{q_{l}}^{+(l)}$ has the form of $(6.3)$ with $q=1, \ldots, 16$, and the superamplitude in the r.h.s.,

$$
\mathcal{A}_{\ldots I_{l} p_{l} \ldots}^{(n)}\left(\ldots ; k_{l}, \theta_{l}^{-} ; \ldots\right):=\mathcal{A}_{\left[I_{1} J_{1} K_{1}\right] \ldots\left[I_{l-1} J_{l-1} K_{l-1}\right] I_{l} p_{l}\left[I_{l+1} J_{l+1} K_{l+1}\right] \ldots}^{(\ldots ;}\left(\ldots ; k_{l}, \theta_{l}^{-} ; \ldots\right),
$$

is fermionic, gamma traceless in $K_{l} p_{l}$ indices,

$$
\gamma_{q_{l} p_{l}}^{I_{l}} \mathcal{A}_{\ldots I_{l} p_{l} \ldots}^{(n)}\left(\ldots ; k_{l}, \theta_{l}^{-} ; \ldots\right)=0,
$$

and is defined by eq. (6.18) itself. The factor $(-)^{\Sigma_{l}}$ can be set to unity in the case under consideration, but we put it in the equation to make it applicable for the case of more general superamplitudes which we discuss below.

Using the suggestion from the on-shell superfield description of the linearized 11D supergravity in eqs. (5.55)-(5.57), we can also introduce a bosonic superfield amplitudes

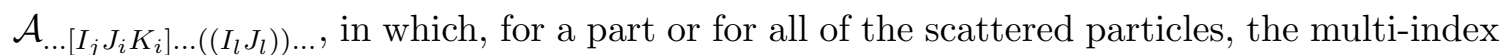
$\left[I_{l} J_{l} K_{l}\right]$, is replaced by a pair of symmetrized traceless indices, $\left(\left(I_{l} J_{l}\right)\right)$. For all $l$ with these new indices we impose on the superamplitude the equation

$$
D_{q_{l}}^{+(l)} \mathcal{A}_{\ldots\left(\left(I_{l} J_{l}\right)\right) \ldots}^{(n)}\left(\ldots ; k_{l}, \theta_{l}^{-} ; \ldots\right)=(-)^{\Sigma_{l}} i \gamma_{\left(I_{l} \mid q_{l} p_{l}\right.} \mathcal{A}_{\left.\ldots \mid J_{l}\right) p_{l} \ldots}^{(n)}\left(\ldots ; k_{l}, \theta_{l}^{-} ; \ldots\right),
$$

with the same fermionic superamplitude obeying (6.20). To be consistent with eqs. (6.18) and (6.21), the fermionic superamplitudes should obey

$$
\begin{aligned}
(-)^{\Sigma_{l}} D_{q_{l}}^{+(l)} \mathcal{A}_{\ldots I_{l} p_{l} \ldots}^{(n)}\left(\ldots ; k_{l}, \theta_{l}^{-} ; \ldots\right) & =2 i \rho_{(l)}^{\#} \gamma_{J_{l} q p} \mathcal{A}_{\ldots\left(\left(I_{l} J_{l}\right)\right) \ldots}^{(n)}\left(\ldots ; k_{l}, \theta_{l}^{-} ; \ldots\right)+ \\
& +\frac{i}{18} \rho_{(l)}^{\#}\left(\gamma_{q p}^{I_{l} J_{l} K_{l} L_{l}}+6 \delta^{I_{l}\left[J_{l}\right.} \gamma_{q p}^{\left.K_{l} L_{l}\right]}\right) \mathcal{A}_{\ldots\left[J_{l} K_{l} L_{l}\right] \ldots}^{(n)}\left(\ldots ; k_{l}, \theta_{l}^{-} ; \ldots\right) .
\end{aligned}
$$

Literally, till now we have discussed superamplitude all the hidden indices of which, denoted by ellipses, are 'bosonic': either antisymmetric triple $\left[I_{j} J_{j} K_{j}\right]$, or symmetric and traceless pair $\left(\left(I_{j} J_{j}\right)\right)$, in which cases $\Sigma_{l}=0$. A more generic amplitude

$$
\mathcal{A}_{Q_{1} \ldots Q_{n}}\left(k_{1}, \theta_{1}^{-} ; k_{2}, \theta_{2}^{-} ; \ldots ; k_{n}, \theta_{n}^{-}\right)=: \mathcal{A}_{\ldots Q_{l} \ldots}\left(\ldots ; k_{l}, \theta_{l}^{-} ; \ldots\right),
$$


can contain for each particle either the bosonic $\left(\left[I_{j} J_{j} K_{j}\right]\right.$ or $\left.\left(\left(I_{j} J_{j}\right)\right)\right)$ or a fermionic multiindex (gamma-traceless pair of one $\mathrm{SO}(9)$ vector and one $\mathrm{SO}(9)$ spinor index, $I_{j} q_{j}$ ),

$$
D=11: \quad Q_{l}=\left\{\left[I_{l} J_{l} K_{l}\right],\left(\left(I_{l} J_{l}\right)\right) ; I_{l} q_{l}\right\}, \quad I_{l}, J_{l}, K_{l}=1, \ldots, 9, \quad q_{l}=1, \ldots, 16 .
$$

Such superfield amplitudes obey eqs. (6.18), (6.21) and (6.22) with generically nontrivial sign factor $(-)^{\Sigma_{l}}: \Sigma_{l}$ can be chosen to be a number of $q_{j}$ indices in the set of indices corresponding to scattered particles with number $j$ in the interval between 1 and $l, 1 \leq j<l$ (see comment in footnote 17),

$$
D=11: \quad \Sigma_{l}=\sum_{j=1}^{l-1} \frac{\left(1-(-)^{\varepsilon\left({ }^{Q_{j}}\right)}\right)}{2}, \quad \varepsilon\left(\left[\left[^{\left[I_{j} J_{j} K_{j}\right]}\right)=0=\varepsilon\left(\left(\left(I_{j} J_{j}\right)\right)\right), \quad \varepsilon\left({ }^{I_{j} q_{j}}\right)=1 .\right.\right.
$$

Sometimes it is instructive to think about Fourier images of the superamplitudes with respect to $\rho_{l}^{\#}$, i.e. dependent on a coordinate $x_{l}^{=}$instead of momentum (energy) $\rho_{l}^{\#}$, e.g.

$$
\mathcal{A}_{\ldots I_{l} q_{l} \ldots}^{(n)}\left(\ldots ; x_{l}^{=}, v_{p l}^{-}, \theta_{p l}^{-} ; \ldots\right)=\int d \rho_{l}^{\#} e^{i x_{l}^{=} \rho_{l}^{\#}} \mathcal{A}_{\ldots I_{l} q_{l} \ldots}^{(n)}\left(\ldots ; \rho_{l}^{\#}, v_{p l}^{-}, \theta_{p l}^{-} ; \ldots\right) .
$$

This superamplitude and its counterparts with a bosonic (multi)index corresponding to $l$-th scattered particle obey the equations $(6.18),(6.21)$ and (6.22) in which $l$-th fermionic covariant derivative $D_{q_{l}}^{+(l)}$ has the form of eq. (6.16).

As in section 4.4, we can formally discuss the action of supersymmetry transformations on the variables corresponding to $l$-th particle only (like (4.25) in the case of 10D SYM); the complete supersymmetry transformation will correspond to simultaneous action of all these $l$-supersymmeteries the parameters of which are expressed through the same constant fermionic spinor parameter as in (4.23). Let us consider the following supersymmetric invariant, which can be treated as an integral over the on-shell superspace corresponding to $l$-th of scattered particles with an exotic superspace measure (cf. (5.62) and see comments and references below (5.48)))

$$
\left.\frac{1}{32} \int d x_{l}^{=}(-)^{\Sigma_{l}} D_{q_{l}}^{+(l)}\left(\mathcal{A}_{\ldots I_{l} q_{l}}^{(l)}\left(\ldots ; x_{l}^{=}, v_{p l}^{-}, \theta_{p l}^{-}\right) \overleftrightarrow{D}_{q_{l}}^{+(l)} \mathcal{A}_{I_{l} q_{l} \ldots}^{(n+2-l)}\left(x_{l}^{=}, v_{p l}^{-}, \theta_{p l}^{-} ; \ldots\right)\right)\right|_{\theta_{q l}^{-}=0}
$$

Using eqs. (6.18), (6.21) and (6.22) we find the equivalent form of this integral:

$$
\begin{aligned}
& -i \int d x_{l}^{=}\left(\mathcal{A}_{\ldots I_{l} q_{l}}^{(l)}\left(\ldots ; x_{l}^{=}, v_{p l}^{-}\right) \overleftrightarrow{\partial}=\mathcal{A}_{I_{l} q_{l} \ldots}^{(n+2-l)}\left(x_{l}^{=}, v_{p l}^{-} ; \ldots\right)\right)+ \\
& +\int d x_{l}^{=} 4 \partial_{=} \mathcal{A}_{\ldots\left(\left(I_{l} J_{l}\right)\right)}^{(l)}\left(\ldots ; x_{l}^{=}, v_{p l}^{-}\right) \partial_{=} \mathcal{A}_{\left(\left(I_{l} J_{l}\right)\right) \ldots}^{(n+2-l)}\left(x_{l}^{=}, v_{p l}^{-} ; \ldots\right)+ \\
& +\int d x_{l}^{=} \frac{1}{3} \partial_{=} \mathcal{A}_{\ldots\left[I_{l} J_{l} K_{l}\right]}^{(l)}\left(\ldots ; x_{l}^{=}, v_{p l}^{-}\right) \partial_{=} \mathcal{A}_{\left[I_{l} J_{l} K_{l}\right] \ldots}^{(n+2-l)}\left(x_{l}^{=}, v_{p l}^{-} ; \ldots\right),
\end{aligned}
$$

which includes the ' $l$-leading components' $\left(\theta_{p(l)}^{-}=0\right.$ value $)$ of the superamplitudes. For the Fourier images of the amplitude the integrand of the above invariant reads

$$
\begin{aligned}
& -2 \mathcal{A}_{\ldots I_{l} q_{l}}^{(l)}\left(\ldots ; \rho_{l}^{\#}, v_{p l}^{-}\right) \rho_{l}^{\#} \mathcal{A}_{I_{l} q_{l} \ldots}^{(n+2-l)}\left(-\rho_{l}^{\#}, v_{p l}^{-} ; \ldots\right)+ \\
& +4 \mathcal{A}_{\ldots\left(\left(I_{l} J_{l}\right)\right)}^{(l)}\left(\ldots ; \rho_{l}^{\#}, v_{p l}^{-}\right)\left(\rho_{l}^{\#}\right)^{2} \mathcal{A}_{\left(\left(I_{l} J_{l}\right)\right) \ldots}^{(n+l)}\left(-\rho_{l}^{\#}, v_{p l}^{-} ; \ldots\right)+ \\
& +\frac{1}{3} \mathcal{A}_{\ldots\left[I_{l} J_{l} K_{l}\right]}^{(l)}\left(\ldots ; \rho_{l}^{\#}, v_{p l}^{-}\right)\left(\rho_{l}^{\#}\right)^{2} \mathcal{A}_{\left[I_{l} J_{l} K_{l}\right] \ldots}^{(n+2-l)}\left(-\rho_{l}^{\#}, v_{p l}^{-} ; \ldots\right) .
\end{aligned}
$$


It is not difficult to check that this is invariant under the following 'component' form of the $l$-th supersymmetry which acts on the $l$-leading components of the superfield amplitudes:

$$
\begin{aligned}
\delta_{\epsilon_{(l)}} \mathcal{A}_{\ldots\left[I_{l} J_{l} K_{l}\right] \ldots}^{\left(n^{\prime}\right)}\left(\ldots ; k_{l} ; \ldots\right) & =(-)^{\Sigma_{l}} 3 i\left(\epsilon \gamma_{\left[J_{l} K_{l} \mid\right.}\right)_{p_{l}} \mathcal{A}_{\left.\ldots \mid I_{l}\right] p_{l} \ldots}^{\left(n^{\prime}\right)}\left(\ldots ; k_{l} ; \ldots\right), \\
\delta_{\epsilon_{(l)}} \mathcal{A}_{\ldots\left(\left(I_{l} J_{l}\right)\right) \ldots}^{\left(n^{\prime}\right)}\left(\ldots ; k_{l} ; \ldots\right) & =(-)^{\Sigma_{l}} i\left(\epsilon \gamma_{\left(I_{l} \mid\right.}\right)_{p_{l}} \mathcal{A}_{\left.\ldots \mid J_{l}\right) p_{l} \ldots}^{\left(n^{\prime}\right)}\left(\ldots ; k_{l} ; \ldots\right) \\
\delta_{\epsilon_{(l)}} \mathcal{A}_{\ldots I_{l} p_{l} \ldots}^{\left(n^{\prime}\right)}\left(\ldots ; k_{l} ; \ldots\right) & \left.\left.=2 i(-)^{\Sigma_{l}} \rho_{l}^{\#}\left(\epsilon \gamma_{J_{l}}\right)_{p_{l}} \mathcal{A}_{\ldots\left(I_{l}\right)}^{\left(n^{\prime}\right)}\left(\ldots . I_{l} J_{l}\right)\right) \ldots ; k_{l} ; \ldots\right)+ \\
+ & \frac{i}{18}(-)^{\Sigma_{l}} \rho_{(l)}^{\#}\left(\epsilon \gamma^{I_{l} J_{l} K_{l} L_{l}}+6 \delta^{I_{l}\left[J_{l}\right.} \epsilon \gamma^{\left.K_{l} L_{l}\right]}\right){ }_{p_{l}} \mathcal{A}_{\ldots\left[J_{l} K_{l} L_{l}\right] \ldots}^{\left(n^{\prime}\right)}\left(\ldots ; k_{l} ; \ldots\right) .
\end{aligned}
$$

The counterpart of (6.28) for the $\rho_{l}^{\#}$ dependent superamplitudes reads

$$
\left.(-)^{\Sigma_{l}} D_{q_{l}}^{+(l)}\left(\mathcal{A}_{\ldots J_{l} p_{l}}^{(l)}\left(\ldots ; k_{l}, \theta_{l}^{-}\right) \overleftrightarrow{D}_{q_{l}}^{+(l)} \mathcal{A}_{J_{l} p_{l} \ldots}^{(n+2-l)}\left(-k_{l}, \theta_{l}^{-} ; \ldots\right)\right)\right|_{\theta_{l}^{-}=0}
$$

where $k_{l}$ is expressed by (4.9) and $D_{q_{l}}^{+(l)}$ has the form of (6.3). This invariant of $l$-th (projection of the) supersymmetry is constructed from two superfield amplitudes including the same variables of one 'internal' particle as the last ( $l$-th) arguments of the first and first arguments of the last superamplitude. The data (variables and indices) of other $l-1$ particles in the first amplitude are generically different from the data of other $(n+1-l)$ particles in the second amplitude. The indices describing 'helicity' of $l$-th particle are fermionic and contracted, $l$-th fermionic variables coincide and $l$-th momentum differs by a sign in two amplitudes. Speaking in terms of energies and spinor moving frame variables, true arguments of the amplitudes, both amplitudes depend on the same $v_{\alpha q(l)}^{-}$but on $+\rho_{l}^{\#}$ and $-\rho_{l}^{\#}$ respectively. Notice that this implies that in the expression (6.3) for $D_{q_{l}}^{+(l)}$ acting on the amplitude with momentum $-k_{l}$ the sign in front of the second term should be also inverted.

The above invariants, as well as their straightforward generalizations including the momentum-dependent multipliers will be useful to describe a candidate for generalization of the BCFW recurrent relations [16] for 11D tree on-shell superamplitudes. But before turning to this, we should understand the $11 \mathrm{D}$ generalization of the $\mathrm{BCFW}$-deformation of the helicity spinors and of the fermionic variables.

\subsection{Generalization of the BCFW deformations for 10D and 11D superamplitudes}

\subsubsection{Bosonic part of $\mathrm{D}=10 \mathrm{BCFW}$ deformation and its 11D generalization}

The $\mathrm{D}=10$ counterparts of the BCFW deformation (2.12), (2.13) of the helicity spinors of the massless particles were presented in [25]. Our spinor moving frame form of the spinor helicity formalism will allow us to simplify them and also to obtain the generalization of the $\mathrm{BCFW}$ deformation to the 11D case. 
As $\mathrm{D}=10$ spinor helicity variables from [25] are identified with spinor frame variables by (4.12), the spinor frame counterparts of the BCFW deformation from [25] has the form

$$
\begin{aligned}
& \widehat{v_{\alpha q n}^{-}}=v_{\alpha q n}^{-}+z \sqrt{\frac{\rho_{1}^{\#}}{\rho_{n}^{\#}}} v_{\alpha p 1}^{-} \mathbb{M}_{p q}, \\
& \widehat{v_{\alpha q 1}^{-}}=v_{\alpha q 1}^{-}-z \sqrt{\frac{\rho_{n}^{\#}}{\rho_{1}^{\#}}} \mathbb{M}_{q p} v_{\alpha p n}^{-},
\end{aligned}
$$

with $\alpha=1, \ldots, 16, \quad q, p=1, \ldots, 8$ and an arbitrary $z \in \mathbb{C}$. The same equation describes also the $11 D$ version of the $B C F W$ deformation provided $\alpha$ and $q, p$ are considered as $\mathrm{SO}(1,10)$ and $\mathrm{SO}(9)$ spinor indices respectively, $\alpha=1, \ldots, 32$ and $q, p=1, \ldots, 16$.

The shift of the spinor frame variables, (6.34) and (6.35) results in shifting the momentum of the first and of the $n$-th particle on a complex light-like vector $q^{a}$ orthogonal to both $k_{(1)}^{a}$ and $k_{(n)}^{a}$,

$$
\begin{aligned}
\widehat{k_{1}^{a}} & =k_{1}^{a}-z q^{a}, & \widehat{k_{n}^{a}} & =k_{n}^{a}+z q^{a}, \\
q_{a} q^{a} & =0, & q_{a} k_{1}^{a} & =0,
\end{aligned}
$$

provided we choose

$$
\mathbb{M}_{q p}=-\frac{1}{\sqrt{\rho_{1}^{\#} \rho_{n}^{\#}}\left(u_{1}^{=} u_{n}^{\bar{n}}\right)}\left(v_{q 1}^{-} \not v_{p n}^{-}\right) .
$$

Here and below

$$
\not^{\alpha \beta}:=q^{a} \tilde{\Gamma}_{a}^{\alpha \beta}, \quad \not q_{\alpha \beta}:=q_{a} \Gamma_{\alpha \beta}^{a} .
$$

It is easy to check that the light-likeness of $q^{a},(6.37)$, implies the nilpotency of the matrix $\mathbb{M}(6.38): \quad \mathbb{M}^{T} \mathbb{M}=0=\mathbb{M M}^{T}$ or more explicitly

$$
\mathbb{M}_{r p} \mathbb{M}_{r q}=0, \quad \mathbb{M}_{q r} \mathbb{M}_{p r}=0 \text {. }
$$

We can also write the expression for light-like complex vector in terms of deformation matrix,

$$
\begin{array}{ll}
D=10: & q^{a}=\frac{1}{4} \sqrt{\rho_{1}^{\#} \rho_{n}^{\#}} v_{q 1}^{-} \tilde{\sigma}^{a} \mathbb{M}_{q p} v_{p n}^{-}, \\
D=11: & q^{a}=\frac{1}{8} \sqrt{\rho_{1}^{\#} \rho_{n}^{\#}} v_{q 1}^{-} \tilde{\Gamma}^{a} \mathbb{M}_{q p} v_{p n}^{-} .
\end{array}
$$

The nilpotency condition (6.40) guarantees that the shifted spinor frame variables obeys the characteristic constraints, eqs. (4.6), (4.9) with shifted light-like momenta $k_{(1)}$ and $k_{(n)}$, (6.36), or equivalently, (3.26) with shifted light-like $u_{(1)}^{=a}$ and $u_{(n)}^{=a}$,

$$
\widehat{u_{1}^{\bar{a}}}=u_{1}^{=a}-\frac{z q^{a}}{\rho_{1}^{\#}}, \quad \widehat{u_{n}^{\overline{=}}}=u_{n}^{=a}+\frac{z q^{a}}{\rho_{n}^{\#}} .
$$


Notice that (6.34) and (6.35) imply

$$
\widehat{k_{1}^{a}}+\widehat{k_{n}^{a}}=k_{1}^{a}+k_{n}^{a}
$$

so that

$$
\widehat{P}_{i j}(z)=\hat{k_{i}}+\ldots+\hat{k_{j}}
$$

is independent of $z$ if both 1 and $n$ or neither of them lay between $i$ and $j$. With our specific choice of two special points to be (1) and (n), this means just that $\widehat{P}_{i j}(z)$ is independent on $z$ if $1<i<j<n$, but keeps $z$ dependence if e.g. $1<j<i<n$.

\subsubsection{Fermionic part of the $\mathrm{D}=10$ and $\mathrm{D}=11 \mathrm{BCFW}$-type deformations}

To wite a candidate for the 10D and 11D generalizations of the BCFW-type recurrent relations $[16,19]$ for our constrained superamplitudes, we need to understand how the BCFW deformations, acting on the 10D and 11D momenta as (6.36), and on the spinor helicity (spinor moving frame) variables as in (6.34) and (6.35), act of the fermionic variables $\theta_{p(i)}^{-}$. The study of Clifford superfield formalism in [25] makes clear that such an action should be nontrivial and actually suggests its possible form:

$$
\begin{aligned}
& \widehat{\theta_{p(n)}^{-}}=\theta_{p(n)}^{-}+z \theta_{q(1)}^{-} \mathbb{M}_{q p} \sqrt{\frac{\rho_{(1)}^{\#}}{\rho_{(n)}^{\#}}}, \\
& \widehat{\theta_{q(1)}^{-}}=\theta_{q(1)}^{-}-z \sqrt{\frac{\rho_{(n)}^{\#}}{\rho_{(1)}^{\#}}} \mathbb{M}_{q p} \theta_{p(n)}^{-}
\end{aligned}
$$

(remember that $q, p=1, \ldots, 8$ for $\mathrm{D}=10$ while $q, p=1, \ldots, 16$ for $\mathrm{D}=11$ ).

This implies the following transformations of the covariant derivatives (6.3):

$$
\begin{aligned}
& \widehat{D_{p(n)}^{+}}=D_{p(n)}^{+}+z D_{q(1)}^{+} \mathbb{M}_{q p} \sqrt{\frac{\rho_{(n)}^{\#}}{\rho_{(1)}^{\#}}}, \\
& \widehat{D_{q(1)}^{+}}=D_{q(1)}^{+}-z \sqrt{\frac{\rho_{(1)}^{\#}}{\rho_{(n)}^{\#}}} \mathbb{M}_{q p} D_{p(n)}^{+} .
\end{aligned}
$$

Due to the nilpotency of the matrix $\mathbb{M},(6.40)$, the shifted derivatives also obey the algebra (6.4),

$$
\left\{\widehat{D}_{q_{l}}^{+(l)}, \widehat{D}_{p_{j}}^{+(j)}\right\}=-2 \delta_{l j} \delta_{q_{l} p_{j}} \rho_{(l)}^{\#} .
$$

It is convenient to introduce a schematic notation

$$
D_{(1)} \mathbb{M} \theta_{(n)}:=\sqrt{\frac{\rho_{(n)}^{\#}}{\rho_{(1)}^{\#}}} D_{q(1)}^{+} \mathbb{M}_{q p} \theta_{q(n)}^{-}, \quad \theta_{(1)} \mathbb{M} D_{(n)}:=\sqrt{\frac{\rho_{(1)}^{\#}}{\rho_{(n)}^{\#}}} \theta_{q(1)}^{-} \mathbb{M}_{q p} D_{p(n)}^{+} .
$$


Then one can equivalently write the definition of the fermionic deformation, (6.46), (6.47), and its consequences, (6.48) and (6.49), in a more universal form

$$
\begin{aligned}
& \widehat{\theta_{p(l)}^{-}}=e^{-z D_{(1)} \mathbb{M} \theta_{(n)}-z \theta_{(1)} \mathbb{M} D_{(n)}} \theta_{p(l)}^{-}, \\
& \widehat{D_{p(l)}^{+}}=e^{-z D_{(1)} \mathbb{M} \theta_{(n)}-z \theta_{(1)} \mathbb{M} D_{(n)} D_{p(l)}^{+} e^{z D_{(1)}} \mathbb{M} \theta_{(n)}+z \theta_{(1)} \mathbb{M} D_{(n)} .}
\end{aligned}
$$

The BCFW deformation of our 10D and 11D superamplitudes

$$
\begin{aligned}
\mathcal{A}_{\ldots}\left(k_{1}, \theta_{1} \ldots, k_{n}, \theta_{1}\right) & =\mathcal{A}_{\ldots}^{(n)}\left(\rho_{(1)}^{\#}, v_{q(1)}^{-}, \theta_{q(1)}^{-} ; \ldots \rho_{(n)}^{\#}, v_{q(n)}^{-}, \theta_{q(n)}^{-}\right) \\
& \equiv \mathcal{A}_{\ldots}^{(n)}\left(\left\{\rho_{(i)}^{\#}\right\} ;\left\{v_{q(i)}^{-}\right\} ;\left\{\theta_{q(i)}^{-}\right\}\right)
\end{aligned}
$$

can be described by the equation

$$
\mathcal{A}_{z \ldots}\left(\ldots, \widehat{k_{(i)}}, \widehat{\theta_{(i)}} \ldots\right)=e^{z D_{(1)} \mathbb{M} \theta_{(n)}+z \theta_{(1)}} \mathbb{M} D_{(n)} \mathcal{A}_{\ldots}\left(\widehat{k_{1}}(z), k_{2}, \ldots, k_{(n-1)}, \widehat{k_{n}}(z)\right) .
$$

\subsection{Candidate BCFW recurrent relations for 10D SYM superamplitudes}

The BCFW shifted $\mathrm{D}=10$ superamplitude (6.54) obeys the superfield equations (6.9) with BCFW shifted fermionic covariant derivatives,

$$
\widehat{D_{q_{l}}^{+(l)}} \mathcal{A}_{z}^{(n)} \ldots \dot{q}_{l} \ldots\left(\ldots ; \widehat{k_{l}}, \widehat{\theta_{l}^{-}} ; \ldots\right)=(-)^{\Sigma_{l}} \gamma_{q_{l} \dot{q}_{l}}^{I_{l}} \mathcal{A}_{z}^{(n)} \ldots I_{l} \ldots\left(\ldots ; \widehat{k_{l}}, \widehat{\theta_{l}^{-}} ; \ldots\right),
$$

or, more schematically,

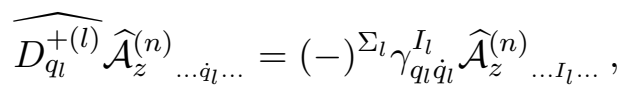

where $\widehat{\mathcal{A}}_{z}^{(n)}{ }_{\ldots I_{l} \ldots}:=\mathcal{A}_{z}^{(n)}{ }_{\ldots I_{l} \ldots}\left(\ldots ; \widehat{k_{l}}, \widehat{\theta_{l}^{-}} ; \ldots\right)$ is related to $\mathcal{A}^{(n)}{ }_{\ldots I_{l} \ldots}\left(\ldots ; \widehat{k}_{l}(z), \theta_{l}^{-} ; \ldots\right)$ by eq. (6.54).

A natural candidate for generalization of the BCFW recursion relations [16] for the case of $10 \mathrm{D}$ tree superfield amplitudes is

$$
\begin{aligned}
& \mathcal{A}_{Q_{1} \ldots Q_{n}}\left(k_{1}, \theta_{(1)}^{-} ; k_{2}, \theta_{(2)}^{-} ; \ldots ; k_{n}, \theta_{(n)}^{-}\right)= \\
& =\sum_{l=2}^{n} \frac{(-)^{\Sigma_{(l+1)}}}{32 \widehat{\rho}^{\#}\left(z_{l}\right)} D_{q\left(z_{l}\right)}^{+}\left(\mathcal{A}_{z_{l}} Q_{1} \ldots Q_{l} \dot{q}\left(\widehat{k_{1}}, \widehat{\theta_{(1)}^{-}} ; k_{2}, \theta_{(2)}^{-} ; \ldots ; k_{l}, \theta_{(l)}^{-} ; \widehat{P}_{l}\left(z_{l}\right), \Theta^{-}\right) \times\right. \\
& \left.\quad \times \frac{1}{\left(P_{l}\right)^{2}} \overleftrightarrow{D}_{q\left(z_{l}\right)}^{+} \mathcal{A}_{z_{l}} \dot{q} Q_{l+1} \ldots Q_{n}\left(-\widehat{P}_{l}\left(z_{l}\right), \Theta^{-} ; k_{l+1}, \theta_{(l+1)}^{-} ; \ldots ; k_{n-1}, \theta_{(n-1)}^{-} ; \widehat{k_{n}}, \widehat{\theta_{(n)}^{-}}\right)\right)\left.\right|_{\Theta^{-}=0} .
\end{aligned}
$$

Let us write the relation (6.57) a bit more explicitly in terms of original amplitudes,

$$
\begin{aligned}
\mathcal{A}_{Q_{1} \ldots Q_{n}}\left(k_{1}, \theta_{1}^{-} ; k_{2}, \theta_{2}^{-} ; \ldots ; k_{n}, \theta_{n}^{-}\right)=\sum_{l=2}^{n} \frac{(-)^{\Sigma_{(l+1)}}}{32 \widehat{\rho}^{\#}\left(z_{l}\right)} \times \\
\quad \times D_{q\left(z_{l}\right)}^{+}\left(e^{z_{l} D_{(1)}} \mathbb{M} \theta_{(n)}+z_{l} \theta_{(1)} \mathbb{M} D_{(n)} \mathcal{A}_{Q_{1} \ldots Q_{l} \dot{q}}\left(\widehat{k_{1}}\left(z_{l}\right), \theta_{1}^{-} ; k_{2}, \theta_{2} ; \ldots ; k_{l}, \theta_{l} ; \widehat{P}_{l}\left(z_{l}\right), \Theta^{-}\right) \times\right. \\
\left.\quad \times \frac{1}{\left(P_{l}\right)^{2}} \overleftrightarrow{D}_{q\left(z_{l}\right)}^{+} \mathcal{A}_{\dot{q} Q_{l+1} \ldots Q_{n}}\left(-\widehat{P}_{l}\left(z_{l}\right), \Theta^{-} ; k_{l+1}, \theta_{l+1} ; \ldots ; \widehat{k_{n}}\left(z_{l}\right), \theta_{l}\right)\right)\left.\right|_{\Theta^{-}=0} .
\end{aligned}
$$


Here

$$
P_{l}^{a}=-\sum_{m=1}^{l} k_{m}^{a}
$$

the deformation parameter is chosen to be

$$
z_{l}:=\frac{P_{l}^{a} P_{l a}}{2 P_{l}^{b} q_{b}}
$$

and

$$
\widehat{P_{l}^{a}}(z)=-\sum_{m=1}^{l} \widehat{k_{m}^{a}}(z)=P_{l}^{a}-2 z \sqrt{\rho_{1}^{\#} \rho_{n}^{\#}} v_{q 1}^{-}{\tilde{\sigma^{a}}}_{\mathbb{M}_{q p}} v_{p n}^{-}=: P_{l}^{a}-z q^{a}
$$

with $v_{q 1}^{-} \tilde{\sigma}^{a} v_{p n}^{-}:=v_{\alpha q_{(1)}}^{-} \tilde{\sigma}^{a \alpha \beta} v_{\beta p(n)}^{-}$and $q_{a}$ defined by eq. (6.41). Eq. (6.61) implies that $\left(\widehat{P}_{l}(z)\right)^{2}=\left(P_{l}\right)^{2}-2 z P_{l} \cdot q$, so that at $z=z_{l}(6.60) \widehat{P_{l}^{a}}$ becomes light-like

$$
\left(\widehat{P}_{l}\left(z_{l}\right)\right)^{2}=0, \quad z_{l}:=\frac{\left(P_{l}\right)^{2}}{2 P_{l} \cdot q}
$$

As a result, both amplitudes in the r.h.s. of (6.57) are on the mass shell. To express them in terms of spinor helicity formalism we introduce spinor frame variables $\widehat{v_{\alpha q}}\left(z_{l}\right)$ related to the light-like $\widehat{P}_{l}^{a}\left(z_{l}\right)$ by the counterpart of eq. (4.6),

$$
\widehat{P_{l}^{a}}\left(z_{l}\right) \sigma_{a \alpha \beta}=2 \widehat{\rho}^{\#}\left(z_{l}\right) \widehat{v_{\alpha q}^{-}}\left(z_{l}\right) \widehat{v_{\beta q}^{-}}\left(z_{l}\right), \quad \widehat{\rho}^{\#}\left(z_{l}\right) \widehat{v_{q}^{-}}\left(z_{l}\right) \tilde{\sigma}^{a} \widehat{v}_{p}^{-}\left(z_{l}\right)=\widehat{P}_{l}^{a}\left(z_{l}\right) \delta_{q p}
$$

Notice that $\widehat{\rho}^{\#}\left(z_{l}\right)$ defined in this equations, enters the denominators of the terms in the r.h.s. of $(6.57)$, in the combination $\frac{(-)^{\Sigma_{(l+1)}}}{32 \widehat{\rho}^{\#}\left(z_{l}\right)}$, where coefficient $1 / 32$ is needed to reproduce the (presumably) correct purely bosonic limit (the counterpart of the relation described for all $\mathrm{D}$ in the introduction of [19]).

Finally, $D_{q\left(z_{l}\right)}^{+}$in (6.57) is the covariant derivative with respect to $\theta_{\dot{q}}^{-}$(in the last argument of first multiplier and first argument of the last multiplier in the r.h.s. of this equation) constructed with the use of $\widehat{\rho}^{\#}\left(z_{l}\right)$,

$$
D_{q\left(z_{l}\right)}^{+}=\frac{\partial}{\partial \theta_{q}^{-}}-2 \widehat{\rho}^{\#}\left(z_{l}\right) \theta_{\dot{q}}^{-}
$$

\subsection{On validity of higher dimensional BCFW relations}

The derivation of the BCFW recurrent relations [16] and their generalizations [19, 95-97] uses essentially the fact that the deformed amplitude as a function of $z$ does not have a pole at $z=\infty$. 
We did not study whether this is the case for our constrained superamplitudes and amplitudes. However, generic D-dimensional results of [19, 95-97] suggest to hope that the above recurrent relations for tree 10D SYM superamplitudes (and the below relations for 11D SUGRA) are valid at least

- in the case if the amplitude in the l.h.s. has at least one vector particle (at least one graviton), which is taken to be the first one, with deformed momentum,

- and after we contract the first vector index $I_{1}$ (first multi-index $\left.\left(I_{1} J_{1}\right)\right)$ ) with the deformation vector $q_{(1)}^{I}$ (7.59) (with direct product of deformation vectors $q_{(1)}^{I} q_{(1)}^{J}$ ).

This latter implies that the deformation 10-vector $q^{a}=u_{1}^{a I} q_{(1)}^{I}$ (direct product of two copies of its $11 \mathrm{D}$ counterpart) is considered to be a polarization vector (tensor) of the scattered vector particle (graviton). This can be always achieved as $q^{a}$ has the properties characteristic for a polarization vector. However, in contradistinction to $4 \mathrm{D}$ case, in higher $D$ there exists an essential freedom in choosing such a deformation vector.

Below, in section 8, we will actually observe a problem related to an essential dependence of the amplitude calculated with BCFW prescription on the deformation vector, and conclude that it might reflect either inconsistency/need for modification of our candidate recurrent relations or incompleteness of our prescription for BCFW deformation. We suggest that this latter might be improved on the line of interplay of our present approach and the analytic superamplitude formalism of [53].

Keeping all these in mind, we nevertheless have written the most generic form of the candidate BCFW relations for 10D SYM (and, below, for 11D SUGRA), with the aim to study their properties and to gain suggestions for further development of the formalism. In particular, below we use them to obtain candidate 4 particle 10D SYM amplitudes with 4 and with 2 fermionic legs which allow us to observe the above mentioned problem of deformation vector dependence.

\subsection{Candidate BCFW recurrent relation for the 11D supergravity amplitudes}

The candidate BCFW-type recurrent relation for tree superfield amplitudes of 11D supergravity reads

$$
\begin{aligned}
& \mathcal{A}_{Q_{1} \ldots Q_{n}}\left(k_{1}, \theta_{(1)}^{-} ; k_{2}, \theta_{(2)}^{-} ; \ldots ; k_{n}, \theta_{(n)}^{-}\right)= \\
& =\sum_{l=2}^{n} \frac{(-)^{\Sigma_{(l+1)}}}{64\left(\widehat{\rho}^{\#}\left(z_{l}\right)\right)^{2}}\left(D _ { q ( z _ { l } ) } ^ { + } \left(\mathcal{A}_{z_{l} Q_{1} \ldots Q_{l} J p}\left(\widehat{k_{1}}, \widehat{\theta_{(1)}^{-}} ; k_{2}, \theta_{(2)}^{-} ; \ldots ; k_{l}, \theta_{(l)}^{-} ; \widehat{P}_{l}\left(z_{l}\right), \theta^{-}\right) \times\right.\right. \\
& \left.\left.\quad \times \frac{1}{\left(P_{l}\right)^{2}} \overleftrightarrow{D}_{q\left(z_{l}\right)}^{+} \mathcal{A}_{z_{l} J p Q_{l+1} \ldots Q_{n}}\left(-\widehat{P}_{l}\left(z_{l}\right), \theta^{-} ; k_{l+1}, \theta_{(l+1)}^{-} ; \ldots ; k_{n-1}, \theta_{(n-1)}^{-} ; \widehat{k_{n}}, \widehat{\theta_{(n)}^{-}}\right)\right)\right)_{\theta_{q}^{-}=0}
\end{aligned}
$$

This looks very much the same as $D=10$ candidate super-BCFW relation (6.57), up to that the superindices take now different values (6.24),

$$
D=11: \quad Q_{l}=\left\{\left[I_{l} J_{l} K_{l}\right],\left(\left(I_{l} J_{l}\right)\right) ; I_{l} q_{l}\right\}, \quad I_{l}, J_{l}, K_{l}=1, \ldots, 9, \quad q_{l}=1, \ldots, 16, \quad(6.66)
$$


so that, in particular, the fermionic multindex of the deformed amplitudes which we sum on is the set of an $\mathrm{SO}(9)$ vector and $\mathrm{SO}(9)$ spinor indices, $I q$ (instead of $8 \mathrm{c}$ index $\dot{q}=1, \ldots, 8$ in the $10 \mathrm{D}$ case).

For the convenience of a reader interested in 11D case only, let us explain the notation, although this is almost identical to the one in 10D case, eqs. (6.59)-(6.64). The reader who feels sufficient the reference to notation desceibed above for 10D case might pass directly to the next section 7 .

In $(6.65)$

$$
P_{l}^{a}=-\sum_{m=1}^{l} k_{m}^{a}
$$

is the (minus) sum of momenta of 'first' $l$ of scattered (super)particles in the first superamplitude,

$$
\widehat{P_{l}^{a}}(z)=-\sum_{m=1}^{l} \widehat{k_{m}^{a}}(z)=P_{l}^{a}-z q^{a}
$$

is the (minus) sum of 'first' $l$ deformed momenta, $q^{a}$ is the deformation vector (6.42) obeying (6.37) as far as the matrix $\mathbb{M}_{q p}$ is nilpotent, (6.40) (see also (6.38)). Finally

$$
z_{l}:=P_{l}^{a} P_{l a} /\left(2 P_{l}^{b} q_{b}\right),
$$

is the value of the deformation parameter $z$ at which $\widehat{P}_{l}^{a}(z)$ becomes light-like,

$$
\left(\widehat{P}_{l}\left(z_{l}\right)\right)^{2}=0, \quad z_{l}:=\left(P_{l}\right)^{2} /\left(2 P_{l} \cdot q\right) .
$$

Thus both amplitudes in the r.h.s. of (6.65) are on the mass shell.

Furthermore, the bosonic arguments of the on-shell amplitudes are energies $\rho_{(i)}^{\#}$ and spinor harmonics $v_{\alpha q(i)}^{-}$related to light-like momenta $k_{(i)}^{a}$ by (4.9); just for shortness in (6.65), we hide this writing instead the dependence on the momenta. In particular, the last bosonic argument of the first amplitudes and the first argument of the second amplitude in the r.h.s. of (6.65) are actually pairs of energy $\pm \widehat{\rho^{\#}}\left(z_{l}\right)$ and spinor frame variables $v_{\alpha q}^{-}\left(z_{l}\right)$ related to $\widehat{P}_{l}^{a}\left(z_{l}\right)$ of (6.68) by eqs. (4.9),

$$
\begin{aligned}
\widehat{P_{l}^{a}}\left(z_{l}\right) \Gamma_{a \alpha \beta} & =2 \widehat{\rho^{\#}}\left(z_{l}\right) v_{\alpha q}{ }^{-}\left(z_{l}\right) v_{\beta q}-\left(z_{l}\right), \\
\widehat{P}_{l}^{a}\left(z_{l}\right) \delta_{q p} & =\widehat{\rho^{\#}}\left(z_{l}\right) v_{q}^{-}\left(z_{l}\right) \tilde{\Gamma}^{a} v_{p}^{-}\left(z_{l}\right) .
\end{aligned}
$$

$\widehat{\rho}^{\#}\left(z_{l}\right)$ defined in this equations enters the denominators of the terms in the r.h.s. of (6.65) with coefficient 64 . This is needed to reproduce a (presumably) correct purely bosonic limit of the candidate BCFW relations in the assumption of simple relation between amplitudes and superamplitudes.

Finally, $D_{q\left(z_{l}\right)}^{+}$in (6.65) is the covariant derivative with respect to $\theta_{q}^{-}$(in the last argument of first multiplier and first argument of the last multiplier in the r.h.s. of (6.65)) constructed with the use of $\widehat{\rho^{\#}}\left(z_{l}\right)$ of $(6.71)$,

$$
D_{q\left(z_{l}\right)}^{+}=\frac{\partial}{\partial \theta_{q}^{-}}-2 \widehat{\rho^{\#}}\left(z_{l}\right) \theta_{q}^{-} .
$$


Notice that the structure of the terms in r.h.s. of (6.65), $D_{q\left(z_{l}\right)}^{+}\left(\mathcal{A}_{z_{l} \ldots J p} \overleftrightarrow{D}_{q\left(z_{l}\right)}^{+} \mathcal{A}_{z_{l} J p \ldots}\right)$ can be treated as an integration over the fermionic variable $\theta_{q}^{-}$in (6.72) with an exotic measure similar to one used in $[92,93]$ to construct a worldsheet superfield formulation of the heterotic string (see [94] for formal discussion of similar exotic measures).

\section{Towards calculation of $11 \mathrm{D}$ and $10 \mathrm{D}$ amplitudes}

In this section we elaborate a bit more the spinor frame form of the spinor helicity formalism and present some details which will be useful for amplitude and superamplitude calculations. A simplest application will be described in the next section 8 for the case of $\mathrm{D}=10 \mathrm{SYM}$ (as we have already noticed, these will indicate some problems of our candidate BCFW recurrent relations). In contrast, the equations of this section are written explicitly for $\mathrm{D}=11$ case, in which $q, p=1, \ldots, 16, I=1, \ldots, 9$. The discrepancy with $\mathrm{D}=10$ case, in which $I=1, \ldots, 8, \quad q, p=1, \ldots, 8$ and $\dot{q}, \dot{p}=1, \ldots, 8$ replace $q$ and $p$ in certain expressions, is described explicitly when it is not evident.

\subsection{Candidate BCFW-type relation for 4-point superamplitudes in $\mathrm{D}=11$}

To gain a feeling of the structure of the candidate recurrent relations (6.65), let us first write it's version for 4-particle (4-supergraviton) tree superamplitude in $\mathrm{D}=11$, selecting 1-st and 4-th particle variables to be deformed,

$$
\begin{aligned}
\mathcal{A}_{Q_{1} Q_{2} Q_{3} Q_{4}}\left(k_{1}, \theta_{(1)}^{-} ; k_{2}, \theta_{(2)}^{-} ; k_{3}, \theta_{(3)}^{-} ; k_{4}, \theta_{(4)}^{-}\right)= \\
=\frac{(-)^{Q_{1}+Q_{2}}}{64\left(\widehat{\rho}^{\#}\left(z_{12}\right)\right)^{2}}\left(D _ { q ( z _ { 1 2 } ) } ^ { + } \left(\mathcal{A}_{z_{12} Q_{1} Q_{2} J p}\left(\widehat{k_{1}}, \widehat{\theta_{(1)}^{-}} ; k_{2}, \theta_{(2)}^{-} ; \widehat{P_{12}}\left(z_{12}\right), \theta^{-}\right) \times\right.\right. \\
\left.\left.\quad \times \frac{1}{\left(P_{12}\right)^{2}} \overleftrightarrow{D}_{q\left(z_{12}\right)}^{+} \mathcal{A}_{z_{12} J p Q_{3} Q_{4}}\left(-\widehat{P_{12}}\left(z_{12}\right), \theta^{-} ; k_{3}, \theta_{(3)}^{-} ; \widehat{k_{4}}, \widehat{\theta_{(4)}^{-}}\right)\right)\right)_{\theta_{q}^{-}=0}+ \\
+\frac{(-)^{Q_{1}+Q_{3}+Q_{2} Q_{3}}}{64\left(\widehat{\rho}^{\#}\left(z_{13}\right)\right)^{2}}\left(D _ { q ( z _ { 1 3 } ) } ^ { + } \left(\mathcal{A}_{z_{12} Q_{1} Q_{3} J p}\left(\widehat{k_{1}}, \widehat{\theta_{(1)}^{-}} ; k_{3}, \theta_{(3)}^{-} ; \widehat{P_{13}}\left(z_{13}\right), \theta^{-}\right) \times\right.\right. \\
\left.\left.\quad \times \frac{1}{\left(P_{13}\right)^{2}} \overleftrightarrow{D}_{q\left(z_{13}\right)}^{+} \mathcal{A}_{z_{13} J p Q_{2} Q_{4}}\left(-\widehat{P_{13}}\left(z_{13}\right), \theta^{-} ; k_{2}, \theta_{(2)}^{-} ; \widehat{k_{4}}, \widehat{\theta_{(4)}^{-}}\right)\right)\right)_{\theta_{q}^{-}=0}
\end{aligned}
$$

Here the multiindices $Q_{i}$ take values (6.24), and the remaining notations are explained in the previous section.

The recurrent relation for the 4-point superamplitude for $D=10$ SYM has the same form as (7.1) but with $1 / 64$ multiplies replaced by $1 / 32, q=1, \ldots, 8$ and the multiindices taking values as in (6.8).

A more explicit form of the relation for 11D superamplitude can be obtained by calculating the action of $D_{q\left(z_{12}\right)}^{+}$derivatives and using the superfield equations (6.18), (6.21) 
and (6.22). For instance for the superamplitude with 4 graviton multi-indices we obtain

$$
\begin{aligned}
& \mathcal{A}_{\left(I_{1} J_{1}\right) \ldots\left(I_{4} J_{4}\right)}\left(k_{1}, \theta_{(1)}^{-} ; k_{2}, \theta_{(2)}^{-} ; k_{3}, \theta_{(3)}^{-} ; k_{4}, \theta_{(4)}^{-}\right)= \\
&=- \mathcal{A}_{z_{12}\left(I_{1} J_{1}\right)\left(I_{2} J_{2}\right) J p}\left(\widehat{k_{1}}, \widehat{\theta_{(1)}^{-}} ; k_{2}, \theta_{(2)}^{-} ; \widehat{\rho^{\#}}\left(z_{12}\right), v_{q}^{-}\left(z_{12}\right), 0\right) \\
& \times \frac{1}{\left(P_{12}\right)^{2} \widehat{\rho}^{\#}\left(z_{12}\right)} \mathcal{A}_{z_{12} J p\left(I_{3} J_{3}\right)\left(I_{4} J_{4}\right)}\left(-\widehat{\rho^{\#}}\left(z_{12}\right), v_{q}^{-}\left(z_{12}\right), 0 ; k_{3}, \theta_{(3)}^{-} ; \widehat{k_{4}}, \widehat{\theta_{(4)}^{-}}\right) \\
&+ 2 \mathcal{A}_{z_{12}\left(I_{1} J_{1}\right)\left(I_{2} J_{2}\right)(I J)}\left(\widehat{k_{1}}, \widehat{\theta_{(1)}^{-}} ; k_{2}, \theta_{(2)}^{-} ; \widehat{\rho^{\#}}\left(z_{12}\right), v_{q}^{-}\left(z_{12}\right), 0\right) \\
& \times \frac{1}{\left(P_{12}\right)^{2}} \mathcal{A}_{z_{12}(I J)\left(I_{3} J_{3}\right)\left(I_{4} J_{4}\right)}\left(-\widehat{\rho^{\#}}\left(z_{12}\right), v_{q}^{-}\left(z_{12}\right), 0 ; k_{3}, \theta_{(3)}^{-} ; \widehat{k_{4}}, \widehat{\theta_{(4)}^{-}}\right) \\
&+ \frac{1}{6} \mathcal{A}_{z_{12}\left(I_{1} J_{1}\right)\left(I_{2} J_{2}\right)[I J K]}\left(\widehat{k_{1}}, \widehat{\theta_{(1)}^{-}} ; k_{2}, \theta_{(2)}^{-} ; \widehat{\rho^{\#}}\left(z_{12}\right), v_{q}^{-}\left(z_{12}\right), 0\right) \\
& \times \frac{1}{\left(P_{12}\right)^{2}} \mathcal{A}_{z_{12}[I J K]\left(I_{3} J_{3}\right)\left(I_{4} J_{4}\right)}\left(-\widehat{\rho^{\#}}\left(z_{12}\right), v_{q}^{-}\left(z_{12}\right), 0 ; k_{3}, \theta_{(3)}^{-} ; \widehat{k_{4}}, \widehat{\theta_{(4)}^{-}}\right) \\
&+(2 \longleftrightarrow 3) .
\end{aligned}
$$

All the superamplitudes in the r.h.s. are superfields as functions of 'external variables' (i.e. depend on two of four $\left.\theta_{q(1)}^{-}, \ldots, \theta_{q(4)}^{-}\right)$but leading component of a complete superamplitude with respect to the 'internal' fermionic variables, i.e. are taken at zero value of the fermionic variable corresponding to the 'internal' line, $\Theta_{q}^{-}=0$.

Let us recall that

- the subindex $z_{12}$ of the superamplitudes indicate the value of the deformation parameter (6.69) used in $\widehat{k_{1}}=\widehat{k_{1}}\left(z_{12}\right), \widehat{\theta_{(1)}^{-}}=\widehat{\theta_{(1)}^{-}}\left(z_{12}\right)$ and $\widehat{k_{4}}=\widehat{k_{4}}\left(z_{12}\right), \widehat{\theta_{(4)}^{-}}=\widehat{\theta_{(4)}^{-}}\left(z_{12}\right)$,

- the arguments $k_{a(i)}$ actually indicate the dependence on corresponding 'energy' and spinor moving frame variables, $\rho_{i}^{\#}$ and $v_{\alpha q(i)}^{-}$;

- in contrast, in the case of 'internal line' the dependence on $\widehat{\rho^{\#}}\left(z_{12}\right)$ and $v_{q}^{-}\left(z_{12}\right)$ (of (6.71) with $P_{l}=P_{12}$ ) is indicated explicitly.

\subsection{On 4-particle and 3-particle amplitudes in $\mathrm{D}=11$}

The superamplitude in the left hand side of (7.2) provides a superfield generalization of the well-known 4-graviton amplitude calculated in 11D directly in [99] and having the structure similar to one which had been known from zero slop limit of type II superstring [101-104]. In our spinor moving frame version of the spinor helicity formalism it reads

$$
\begin{aligned}
& \mathcal{A}_{\left(\left(I_{1} J_{1}\right)\right) \ldots\left(\left(I_{4} J_{4}\right)\right)}\left(k_{1}, k_{2}, k_{3}, k_{4}\right)=-\frac{4 \kappa^{2}}{s t u} K^{\left(\left(I _ { 1 } | _ { 1 } \ldots \left(\left(\left.I_{4}\right|_{4}\right.\right.\right.\right.} K^{\left.\left.\left.\left.\mid J_{1}\right)\right)_{1} \ldots \mid J_{4}\right)\right)_{4}}= \\
& -\frac{4 \kappa^{2}}{s t u} t_{8}^{a_{1} \ldots a_{8}} t_{8}^{b_{1} \ldots b_{8}} k_{a_{1}(1)} u_{a_{2}(1)}^{\left(\left(\left.I_{1}\right|_{1}\right.\right.} \ldots k_{a_{7}(4)} u_{a_{8}(4)}^{\left(\left(\left.I_{4}\right|_{4}\right.\right.} k_{b_{1}(1)} u_{b_{2}(1)}^{\left.\left.\mid J_{1}\right)\right)_{1}} \ldots k_{b_{7}(4)} u_{b_{8}(4)}^{\left.\left.\mid J_{4}\right)\right)_{4}},
\end{aligned}
$$

where

$$
k_{a(i)}=\rho_{(i)}^{\#} u_{a(i)}^{=},
$$


$s, t, u$ are standard Mandelstam variables (see below for their expression in moving frame formalism),

$$
\begin{aligned}
K^{I_{1} \ldots I_{4}} & =t_{8}^{a_{1} \ldots a_{8}} k_{a_{1}(1)} u_{a_{2}(1)}^{I_{1}} \ldots k_{a_{7}(4)} u_{a_{8}(4)}^{I_{4}} \\
& =\rho_{(1)}^{\#} \ldots \rho_{(4)}^{\#} t_{8}^{a_{1} \ldots a_{8}} u_{a_{1}(1)}^{=} u_{a_{2}(1)}^{I_{1}} \ldots u_{a_{7}(4)}^{=} u_{a_{8}(4)}^{I_{4}}
\end{aligned}
$$

and tensor $t_{8}$ is defined in $[101,102]$. A compact expression for this tensor is

$$
\begin{aligned}
& t_{8}^{a_{1} \ldots a_{8}} F_{a_{1} a_{2}}^{(1)} \ldots F_{a_{7} a_{8}}^{(4)}=\frac{1}{3}\left(\operatorname{tr}\left(F^{(1)} F^{(2)} F^{(3)} F^{(4)}\right)+(2 \leftrightarrow 3)+(1 \leftrightarrow 4)\right)- \\
& -\frac{1}{12}\left(\operatorname{tr}\left(F^{(1)} F^{(2)}\right) \operatorname{tr}\left(F^{(3)} F^{(4)}\right)+\operatorname{tr}\left(F^{(1)} F^{(3)}\right) \operatorname{tr}\left(F^{(2)} F^{(4)}\right)+\operatorname{tr}\left(F^{(1)} F^{(2)}\right) \operatorname{tr}\left(F^{(3)} F^{(4)}\right)\right) .
\end{aligned}
$$

where $F_{a_{1} a_{2}}^{(1)}, \ldots, F_{a_{7} a_{8}}^{(4)}$ are arbitrary antisymmetric tensor fields.

It is also instructive to compare (7.3) with the $D=10 \mathrm{SYM} 4$-gluon tree amplitude [101-103]. Omitting the color factor $\left(\operatorname{Tr}\left(\mathrm{T}_{1} \mathrm{~T}_{2} \mathrm{~T}_{3} \mathrm{~T}_{4}\right)\right.$ in [104]) it reads

$$
\begin{aligned}
\mathcal{A}_{\check{I} \check{J} \check{K} \check{L}}^{10 D} & =-\frac{2 g^{2}}{s t} K^{\check{I}_{1} \check{I}_{2} \check{I}_{3} \check{I}_{4}}=-\frac{2 g^{2}}{s t} \rho_{(1)}^{\#} \ldots \rho_{(4)}^{\#} t_{8}^{a_{1} \ldots a_{8}} u_{a_{1}(1)}^{=} u_{a_{2}(1)}^{\check{I}} \ldots u_{a_{7}(4)}^{=} u_{a_{8}(4)}^{\check{L}}, \\
\check{I}, \check{J}, \check{K}, \check{L} & =1, \ldots, 8 .
\end{aligned}
$$

Here and below in this section we have denoted the $\mathrm{SO}(8)$ vector indices by hatted symbols, $\breve{I}=1, \ldots, 8$, to distinguish it from $\mathrm{SO}(9)$ indices, $I, J=1, \ldots, 9$ often situated close.

To make the expression (7.7) more illustrative, one has to use (7.6) with the on-shell field strengths (4.16),

$$
F_{a b}^{\check{I}(i)}=k_{[a \mid(i)} u_{\mid b](i)}^{\check{I}}=\rho_{(i)}^{\#} u_{[a \mid(i)} u_{\mid b](i)}^{\check{I}}, \quad \check{I}=1, \ldots, 8,
$$

and writes $(7.7)$ as $\mathcal{A}_{\check{I}_{1} \check{I}_{2} \check{I}_{3} \check{I}_{4}}^{10 D}=-\frac{2 g^{2}}{s t} t_{8}^{a_{1} \ldots a_{8}} F_{a_{1} a_{2} 1}^{\check{I}_{1}} \ldots F_{a_{7} a_{8} 4}^{\check{I}_{4}}$. In string theory (the 8dimensional version of) this comes from the path integral over zero modes of fermionic variables which can be described by $\mathrm{SO}(8)$ spinors $\eta_{\dot{q}}, \dot{q}=1, \ldots, 8$,

$$
t_{8}^{\check{I}_{1} \ldots \check{I}_{8}} F_{\check{I}_{1} \check{I}_{2}}{ }^{(1)} \ldots F_{\check{I}_{7} \check{I}_{8}}{ }^{(4)}=\propto \int d^{8} \eta_{\dot{q}} \exp \left\{\sum_{i=1}^{4} \eta \tilde{\gamma}^{\check{I}_{i} \check{J}_{i}} \eta F_{\check{I}_{1} \check{I}_{2}}{ }^{(i)}\right\},
$$

[104] where $\tilde{\gamma}_{\dot{q} \dot{p}}^{\check{I} \breve{p}}=\gamma_{q \dot{q}}^{[\check{I}} \gamma_{q \dot{p}}^{\breve{J}]}$ and $\gamma_{q \dot{p}}^{\check{I}}$ are $\mathrm{SO}(8)$ Klebsh-Gordan coefficients, $\check{I}, \check{J}=1, \ldots, 8$.

It is natural to assume that $(7.3)$ is given by leading $\left(\theta_{q(1)}^{-}=0, \ldots, \theta_{q(4)}^{-}=0\right)$ component of (7.2). Then (7.3) should be reproducible form the following recurrent relation

$$
\begin{aligned}
& \mathcal{A}_{\left(I_{1} J_{1}\right) \ldots\left(I_{4} J_{4}\right)}\left(k_{1}, k_{2}, k_{3}, k_{n}\right)= \\
&=2 \mathcal{A}_{z_{12}\left(I_{1} J_{1}\right)\left(I_{2} J_{2}\right)(I J)}\left(\widehat{k_{1}}, k_{2} ; \widehat{\rho^{\#}}\left(z_{12}\right), v_{q}^{-}\left(z_{12}\right)\right) \\
& \times \frac{1}{\left(P_{12}\right)^{2}} \mathcal{A}_{z_{12}(I J)\left(I_{3} J_{3}\right)\left(I_{4} J_{4}\right)}\left(-\widehat{\rho^{\#}}\left(z_{12}\right), v_{q}^{-}\left(z_{12}\right) ; k_{3}, \widehat{k_{4}}\right) \\
&+ \frac{1}{6} \mathcal{A}_{z_{12}\left(I_{1} J_{1}\right)\left(I_{2} J_{2}\right)[I J K]}\left(\widehat{k_{1}}, k_{2} ; \widehat{\rho^{\#}}\left(z_{12}\right), v_{q}^{-}\left(z_{12}\right)\right) \\
& \times \frac{1}{\left(P_{12}\right)^{2}} \mathcal{A}_{z_{12}[I J K]\left(I_{3} J_{3}\right)\left(I_{4} J_{4}\right)}\left(-\widehat{\rho^{\#}}\left(z_{12}\right), v_{q}^{-}\left(z_{12}\right) ; k_{3}, \widehat{k_{4}}\right) \\
&+(2 \longleftrightarrow 3) .
\end{aligned}
$$


In writing this we have used the evident fact that the amplitude with odd number of fermions vanishes, in particular $\mathcal{A}_{z_{12}\left(I_{1} J_{1}\right)\left(I_{2} J_{2}\right) J p}\left(\widehat{k_{1}}, k_{2}, P_{12}\right) \equiv 0$ (which is not correct in the case of superamplitudes).

Furthermore it is reasonable to assume that $\mathcal{A}_{z_{12}\left(I_{1} J_{1}\right)\left(I_{2} J_{2}\right)[I J K]}\left(\widehat{k_{1}}, k_{2} ; P_{12}\right)$ also vanishes as otherwise it would produce after dimensional reduction, a nonvanishing amplitude of creation of a single scalar particle in the collision of two gravitons; even with complex momenta the existence of such an amplitude is counterintuitive. Using this hypothesis to omit the second term and its $(2 \leftrightarrow 3)$ counterpart, we arrive at the pure bosonic BCFW relation for four graviton amplitudes

$$
\begin{aligned}
& \mathcal{A}_{\left(I_{1} J_{1}\right) \ldots\left(I_{4} J_{4}\right)}\left(k_{1}, k_{2}, k_{3}, k_{n}\right)= \\
& =2 \mathcal{A}_{z_{12}\left(I_{1} J_{1}\right)\left(I_{2} J_{2}\right)(I J)}\left(\widehat{k_{1}}, k_{2} ; \widehat{\rho^{\#}}\left(z_{12}\right), v_{q}^{-}\left(z_{12}\right)\right) \\
& \quad \times \frac{1}{\left(P_{12}\right)^{2}} \mathcal{A}_{z_{12}(I J)\left(I_{3} J_{3}\right)\left(I_{4} J_{4}\right)}\left(-\widehat{\rho^{\#}}\left(z_{12}\right), v_{q}^{-}\left(z_{12}\right) ; k_{3}, \widehat{k_{4}}\right) \\
& +2 \mathcal{A}_{z_{12}\left(I_{1} J_{1}\right)\left(I_{3} J_{3}\right)(I J)}\left(\widehat{k_{1}}, k_{3} ; \widehat{\rho^{\#}}\left(z_{13}\right), v_{q}^{-}\left(z_{13}\right)\right) \\
& \quad \times \frac{1}{\left(P_{13}\right)^{2}} \mathcal{A}_{z_{13}(I J)\left(I_{2} J_{2}\right)\left(I_{4} J_{4}\right)}\left(-\widehat{\rho^{\#}}\left(z_{13}\right), v_{q}^{-}\left(z_{13}\right) ; k_{2}, \widehat{k_{4}}\right) .
\end{aligned}
$$

In different formalism such type relations in a gravity field theory at arbitrary $D$ were discussed in [19]

Three graviton amplitude is known to be [105] (in [103] one can find also the $\alpha^{\prime}$ correction to this tensor)

$$
\begin{aligned}
& \mathcal{A}_{\left(\left(I_{1} J_{1}\right)\right)\left(\left(I_{2} J_{2}\right)\right)\left(\left(I_{3} J_{3}\right)\right)}\left(k_{1}, k_{2}, k_{3}\right)=\kappa t^{\left(\left(I _ { 1 } | _ { 1 } \left(\left(I _ { 2 } | _ { 2 } \left(\left(\left.I_{3}\right|_{3}\right.\right.\right.\right.\right.\right.} t^{\left.\left.\left.\left.\left.\left.\mid J_{1}\right)\right)_{1} \mid J_{3}\right)\right)_{3} \mid J_{3}\right)\right)_{3}} \\
& \quad=\kappa t^{a_{1} a_{2} a_{3}}\left(k_{1}, k_{2}, k_{3}\right) t^{b_{1} b_{2} b_{3}}\left(k_{1}, k_{2}, k_{3}\right) u_{a_{1}(1)}^{\left(\left(I_{1}\right.\right.} u_{b_{1}(1)}^{\left.\left.J_{1}\right)\right)} u_{a_{2}(2)}^{\left(\left(I_{2}\right.\right.} u_{b_{2}(2)}^{\left.\left.J_{2}\right)\right)} u_{a_{3}(3)}^{\left(\left(I_{3}\right.\right.} u_{b_{3}(3)}^{\left.\left.J_{3}\right)\right)}
\end{aligned}
$$

where

$$
\begin{aligned}
t^{I_{1} I_{2} I_{3}} & =t^{a b c}\left(k_{1}, k_{2}, k_{3}\right) u_{a(1)}^{I_{1}} u_{b(2)}^{I_{2}} u_{c(3)}^{I_{3}} \\
& =\left(k_{(2)} u_{(1)}^{I_{1}}\right)\left(u_{(2)}^{I_{2}} u_{(3)}^{I_{3}}\right)+\left(k_{(3)} u_{(2)}^{I_{2}}\right)\left(u_{(1)}^{I_{1}} u_{(3)}^{I_{3}}\right)+\left(k_{(1)} u_{(3)}^{I_{3}}\right)\left(u_{(1)}^{I_{1}} u_{(2)}^{I_{2}}\right),
\end{aligned}
$$

and

$$
t^{a b c}\left(k_{1}, k_{2}, k_{3}\right)=k_{(2)}^{a} \eta^{b c}+k_{(3)}^{b} \eta^{a c}+k_{(1)}^{c} \eta^{a b} .
$$

Notice the cyclic symmetry property of the t-tensor: $t^{a b c}\left(k_{1}, k_{2}, k_{3}\right)=t^{b c a}\left(k_{2}, k_{3}, k_{1}\right)$.

The structure of the 'Chern-Simons' term of the 11D supergravity action and the form of the on-shell field strength of the 3-form gauge field (called 'formon' in [99]), $F_{a b c d}^{I J K}=$ $k_{[a} u_{b}^{I} u_{c}^{J} u_{d]}^{K}$ (4.19), suggests the following structure of the 'three formon' amplitude

$$
\begin{aligned}
& \mathcal{A}_{\left[I_{1} J_{1} K_{1}\right]\left[I_{2} J_{2} K_{2}\right]\left[I_{3} J_{3} K_{3}\right]}\left(k_{1} ; k_{2} ; k_{3}\right)= \\
& \propto \epsilon^{a b c_{1} c_{2} \ldots c_{8} c_{9}} k_{1 a} k_{2 b} u_{c_{1}(1)}^{I_{1}} u_{c_{2}(1)}^{J_{1}} u_{c_{3}(1)}^{K_{1}} u_{c_{4}(2)}^{I_{2}} u_{c_{5}(2)}^{J_{2}} u_{c_{6}(2)}^{K_{2}} u_{c_{7}(3)}^{I_{3}} u_{c_{8}(3)}^{J_{3}} u_{c_{9}(3)}^{K_{3}} .
\end{aligned}
$$

To check that this amplitude possess the cyclic symmetry property characteristic for the bosonic particles $\mathcal{A}(1,2,3)=\mathcal{A}(3,1,2)=\mathcal{A}(2,3,1)$, we have to take into account the 
momentum conservation $k_{a 1}+k_{a 2}+k_{a 3}=0$. It is also, symmetric under exchange of complete sets of two particle data, e.g. it implies $\mathcal{A}_{\left[I_{1} J_{1} K_{1}\right]\left[I_{2} J_{2} K_{2}\right]\left[I_{3}, J_{3}, K_{3}\right]}\left(k_{1} ; k_{2} ; k_{3}\right)=$ $\mathcal{A}_{\left[I_{2} J_{2} K_{2}\right]\left[I_{1} J_{1} K_{1}\right]\left[I_{3} J_{3} K_{3}\right]}\left(k_{2} ; k_{1} ; k_{3}\right)$, etc.

Some amplitudes of the 10D SYM will be discussed in the next section 8 .

The above 11D discussion makes transparent that the bosonic amplitudes are expressed through contractions of the vector harmonics describing the frames associated with different particles, typically $u_{a i}^{=} u_{j}^{a J}$ and $u_{a i}^{I} u_{j}^{a J}$; similarly, the fermionic amplitudes involve contractions of spinor harmonics from different spinor frames.

Thus, to proceed with amplitude calculations in our formalism, we should understand better the relation between spinor frames associated to different particles. Below we address this problem, present an explicit parametrization of $j$-th frame in term of $i$-th frame and a number of parameters, and also find a gauge fixing conditions for the auxiliary gauge symmetries acting on the spinor frames, which makes the relation of different frames especially simple.

\subsection{Relation between spinor frames associated to different external particles}

For a possible reader convenience, let us begin this section by recalling that, in our spinor frame form of the spinor helicity formalism, the light-like external 11D momenta $k_{i}^{a}$ are expressed thought bilinear of spinor helicity variables, $\lambda_{\alpha q i}=\sqrt{\rho_{i}^{\#}} v_{\alpha q i}^{-}$,

$$
k_{i}^{a}=\rho_{i}^{\#} u_{i}^{a=}, \quad u_{i}^{a=}=\frac{1}{16} v_{q i}^{-} \Gamma^{a} v_{q i}^{-},
$$

where $v_{\alpha q(i)}^{-}$obey the constraints

$$
u_{a i}^{=} \Gamma_{\alpha \beta}^{a}=2 v_{\alpha q i}^{-} v_{\beta q i}^{-}, \quad v_{q i}^{-} \tilde{\Gamma}_{a} v_{p i}^{-}=u_{a}^{=} \delta_{q p}
$$

and are considered as the spinor frame variables (or Lorentz harmonics) (4.4), related to vector frame (vector harmonics) (4.2), (3.11)-(3.13), by (3.27)-(3.30) (with $q=1, \ldots, 8$, $\dot{q}=1, \ldots, 8, I=1, \ldots, 8$ when $\mathrm{D}=10$ and $\dot{q}=q=1, \ldots, 16, I=1, \ldots, 9$ when $\mathrm{D}=11)$.

To proceed with clarification of the structure of (7.1) and similar amplitudes, we need to describe the relations between spinor frame variables associated to different particles.

For the spinor helicity variables this problem might seem to be difficult, but knowing its relation to Lorentz harmonics and using the group theoretical meaning of these one can solve it. Indeed, as Lorentz harmonics are elements of $\operatorname{Spin}(1, D-1)$ group valued matrix (4.4), they are related with another set of Lorentz harmonics by an $\operatorname{Spin}(1, D-1)$ transformations. Allowing for both positive and negative values of the 'energy' variables $\rho_{i}^{\#}$ and $\rho_{j}^{\#}$, we can write the relation between the harmonics describing spinor frames of $j$-th and $i$-th 11D particles in the form

$$
\begin{aligned}
& v_{\alpha q j}^{-}=e^{-\beta_{j i}} \mathcal{O}_{j i q p}\left(v_{\alpha p i}^{-}+\frac{1}{2} K_{j i}^{=I} \gamma_{p p^{\prime}}^{I} v_{\alpha p^{\prime} i}^{+}\right), \\
& v_{\alpha q j}^{+}=e^{\beta_{j i}} \mathcal{O}_{j i q p} v_{\alpha p i}^{+}+\frac{1}{2} K_{j i}^{\# I} v_{\alpha p(j)}^{-} \gamma_{p q}^{I} .
\end{aligned}
$$


In these equations $\mathcal{O}_{(j i) q p}$ is an element of $\operatorname{Spin}(9)(\subset \operatorname{Spin}(1,10))$, i.e. $16 \times 16$ matrix which obeys $^{18}$

$$
\mathcal{O}_{j i q r} \mathcal{O}_{j i p s} \gamma_{r s}^{I}=\gamma_{q p}^{J} \mathcal{O}_{j i}^{J I},
$$

where $9 \times 9$ matrix $\mathcal{O}_{(j i)}^{J I}$ belongs to $\mathrm{SO}(9)$ group. The scale factor $e^{-\beta_{j i}}$ is considered as an element of $\mathrm{SO}(1,1) \subset \mathrm{SO}(1,10)$. It is instructive to calculate the contractions

$$
\begin{aligned}
v_{p i}^{-\alpha} v_{\alpha q j}^{-} & =-\frac{1}{2} e^{-\beta_{i j}} K_{i j}^{=I}\left(\mathcal{O}_{i j} \gamma^{I}\right)_{p q}=\frac{1}{2} e^{-\beta_{j i}} K_{j i}^{=I}\left(\mathcal{O}_{i j} \gamma^{I}\right)_{q p}, \\
v_{p i}^{+\alpha} v_{\alpha q j}^{-} & =e^{+\beta_{i j}} \mathcal{O}_{i j p p^{\prime}}\left(\delta_{p^{\prime} q}+\frac{1}{4} \tilde{K}_{i j}^{\# I} K_{i j}^{=I}\left(\gamma^{I} \gamma^{J}\right)_{p^{\prime} q}\right)=e^{-\beta_{j i}} \mathcal{O}_{j i p q}, \\
\tilde{K}_{j i}^{\# I} & =K_{j i}^{\# J} \mathcal{O}_{j i}^{J I} e^{-2 \beta_{j i}} .
\end{aligned}
$$

Eqs. (7.20) and (7.22) are written for 11D case, while their 10D counterparts carry dotted spinor indices,

$$
\begin{aligned}
& D=10 \text { ： } \\
& v_{\alpha \dot{q} j}^{+}=e^{\beta_{j i}} \tilde{\mathcal{O}}_{j i \dot{q} \dot{p}}\left(v_{\alpha p^{\prime} i}^{+}\left(\delta_{\dot{p}^{\prime} \dot{p}}+\frac{1}{4} K_{j i}^{=I} K_{j i}^{\# J}\left(\tilde{\gamma}^{I} \gamma^{J}\right)_{\dot{p}^{\prime} \dot{p}}\right)+\frac{1}{2} K_{j i}^{\# I} v_{\alpha p^{\prime} i}^{-} \gamma_{p^{\prime} \dot{p}}^{I}\right), \\
& v_{\dot{p} i}^{-\alpha} v_{\alpha q j}^{-}=-\frac{1}{2} e^{-\beta_{i j}} K_{i j}^{=I}\left(\tilde{\mathcal{O}}_{i j} \tilde{\gamma}^{I}\right)_{\dot{p} q}=\frac{1}{2} e^{-\beta_{j i}} K_{j i}^{=I}\left(\mathcal{O}_{i j} \gamma^{I}\right)_{q \dot{p}}, \\
& \mathcal{O}_{j i q p} \tilde{\mathcal{O}}_{j i \dot{q} \dot{p}} \gamma_{q \dot{q}}^{I}=\gamma_{q \dot{q}}^{J} \mathcal{O}_{j i}^{J I}
\end{aligned}
$$

For the vector harmonics we have the following relations

$$
\begin{aligned}
u_{a j}^{=}= & e^{-2 \beta_{j i}}\left(u_{a i}^{=}+\frac{1}{4}\left(\vec{K}_{j i}^{=}\right)^{2} u_{a i}^{\#}+K_{j i}^{=I} u_{a i}^{I}\right) \\
u_{a j}^{\#}= & e^{+2 \beta_{j i}}\left(u_{a i}^{\#}\left(1+\frac{1}{2} K_{j i}^{=I} \tilde{K}_{j i}^{\# I}+\frac{1}{16}\left(\vec{K}_{j i}^{\bar{y}}\right)^{2}\left(\vec{K}_{j i}^{\#}\right)^{2}\right)+\frac{1}{4} u_{a i}^{=}\left(\tilde{K}_{j i}^{\# I}\right)^{2}+\right. \\
& \left.+u_{a(i)}^{I}\left(\tilde{K}_{j i}^{\# I}+\frac{1}{4} K_{j i}^{=I}\left(\vec{K}_{j i}^{\#}\right)^{2}\right)\right) \\
u_{a j}^{I}= & \left(u_{a i}^{K}\left(\delta^{K J}+\frac{1}{2} K_{j i}^{=K} \tilde{K}_{j i}^{\# J}\right)+\right. \\
& +\frac{1}{2} u_{a i}^{\#}\left(K_{j i}^{=J}+\frac{1}{4} \tilde{K}_{j i}^{\# J}\left(\vec{K}_{j i}^{\bar{j}}\right)^{2}\right)+\frac{1}{2} u_{a i}^{\left.=\tilde{K}_{j i}^{\# J}\right) \mathcal{O}_{j i}^{J I} .}
\end{aligned}
$$

where $\left(\overrightarrow{K_{j i}}\right)^{2}=K_{j i}^{\overline{=}} K_{j i}^{\overline{=}}$ and $\mathcal{O}_{j i}^{J I} \in \mathrm{SO}(9)(\in \mathrm{SO}(8)$ for $\mathrm{D}=10)$ is defined in (7.21) ((7.27) for $\mathrm{D}=10)$. Finally $K_{(j i)}^{\# J}$ is the parameter of $K_{(D-2)}$ symmetry and $K_{(j i)}^{=I}$ parametrize the coset $\frac{\mathrm{SO}(1, D-1)}{[\mathrm{SO}(1,1) \otimes \mathrm{SO}(D-2)] \otimes K_{(D-2)}}$.

The above equations simplify essentially if we fix the gauge with respect to $K_{(D-2) i}$ symmetries acting on different spinor frames (4.4) by setting

$$
K_{j i}^{\# I}=0 .
$$

\footnotetext{
${ }^{18}$ Notice that the second term in (7.20) contains $v_{\alpha p(j)}^{-}$. A more explicit expression in terms of $v_{\alpha p i}^{ \pm}$reads $v_{\alpha q j}^{+}=e^{\beta_{j i}} \mathcal{O}_{j i q p}\left(v_{\alpha p^{\prime} i}^{+}\left(\delta_{p^{\prime} p}+\frac{1}{4} K_{j i}^{=I} \tilde{K}_{j i}^{\# J}\left(\gamma^{I} \gamma^{J}\right)_{p^{\prime} p}\right)+\frac{1}{2} \tilde{K}_{j i}^{\# I} v_{\alpha p^{\prime}(i)}^{-} \gamma_{p^{\prime} p}^{I}\right)$ with $\tilde{K}_{(j i)}^{\# I}=K_{j i}^{\# J} \mathcal{O}_{j i}^{J I} e^{-2 \beta_{j i}}$ $($ see $(7.24))$.
} 
To be convinced that this is possible, one should notice that, say, $K_{j i}^{\# I}=K_{j 1}^{\# I}-K_{i 1}^{\# I}$ so that the set of independent relations in (7.31) can be chosen to be $K_{j 1}^{\# I}=0$. In the gauge (7.31) the relations between sets of vector harmonics (7.28)-(7.30) simplify to

$$
\begin{aligned}
& u_{a j}^{=}=e^{-2 \beta_{j i}}\left(u_{a i}^{=}+\frac{1}{4}\left(\vec{K}_{j i}^{\bar{E}}\right)^{2} u_{a i}^{\#}+K_{j i}^{=I} u_{a i}^{I}\right), \\
& u_{a j}^{\#}=e^{+2 \beta_{j i}} u_{a i}^{\#}, \\
& u_{a j}^{I}=\left(u_{a i}^{J}+\frac{1}{2} \vec{K}_{j i}^{\bar{E}} u_{a i}^{\#}\right) \mathcal{O}_{j i}^{J I},
\end{aligned}
$$

and (7.20) acquires the form

$$
v_{\alpha q j}^{+}=e^{\beta_{j i}} \mathcal{O}_{j i q p} v_{\alpha p i}^{+} .
$$

For completeness, let us write explicitly also the gauge fixed expression for the complete set of 10D Lorentz harmonics

$$
\begin{aligned}
& v_{\alpha q j}^{-}=e^{-\beta_{j i}} \mathcal{O}_{(j i) q p}\left(v_{\alpha p i}^{-}+\frac{1}{2} K_{j i}^{=I} \gamma_{p \dot{p}}^{I} v_{\alpha \dot{p} i}^{+}\right), \\
& v_{\alpha \dot{q} j}^{+}=e^{\beta_{j i}} \mathcal{O}_{j i \dot{q} \dot{p}} v_{\alpha \dot{p} i}^{+},
\end{aligned}
$$

and for the elements of the inverse spinor frame matrix (3.23),

$$
\begin{aligned}
v_{\dot{q} j}^{-\alpha} & =e^{-\beta_{j i}} \mathcal{O}_{j i \dot{q} \dot{p}}\left(v_{\dot{p} i}^{-\alpha}-\frac{1}{2} K_{j i}^{=I} v_{\alpha p i}^{+\alpha} \gamma_{p \dot{p}}^{I}\right), \\
v_{q j}^{+\alpha} & =e^{\beta_{j i}} \mathcal{O}_{j i q p} v_{p i}^{+\alpha} .
\end{aligned}
$$

In the next section 7.4 we consider stronger gauge fixing conditions for the auxiliary gauge symmetries acting on the spinor frame variables, in which these are expressed through the $(D-2)$ parameters $K_{j i}^{=I}$ only.

\subsection{Reference spinor frame and complete gauge fixing of the auxiliary gauge symmetries}

It is convenient to introduce an auxiliary spinor frame $\left(v_{\alpha q}^{-}, v_{\alpha q}^{+}\right)$and associated vector frame $\left(u_{a}^{\bar{E}}, u_{a}^{\#}, u_{a}^{I}\right)$. Any of the spinor harmonics $\left(v_{\alpha q j}^{-}, v_{\alpha q j}^{+}\right)$and associated vector harmonics $\left(u_{a j}^{=}, u_{a j}^{\#}, u_{a j}^{I}\right)$ are related with reference spinor frame and reference frame by (7.19), (7.20), (7.28), (7.29), (7.30) with omitted index $i$. Then they are parametrized by the set of $K_{j}^{=I}, e^{-\beta_{j}}, \mathcal{O}_{j}^{I J}=\left(\mathcal{O}_{j}\right)^{-1 J I}, K_{j}^{\# I}$, in which the last three subsets of parameters correspond to the gauge symmetry transformations. These are used as identification relations which allow to consider the sets of harmonic variables $\left(v_{\alpha q(i)}^{-}, v_{\alpha q(i)}^{+}\right)$as homogeneous coordinates of the celestial sphere. We call them auxiliary gauge symmetries and can conventionally fix them by setting

$$
K_{i}^{\# I}=0, \quad \mathcal{O}_{i}^{I J}=\delta^{I J}, \quad e^{-\beta_{i}}=1 .
$$


Then any 11D spinor frame can be expressed through the auxiliary spinor frame by

$$
v_{\alpha q(i)}^{-}=v_{\alpha q}^{-}+\frac{1}{2} K_{i}^{=I} \gamma_{q p}^{I} v_{\alpha p}^{+}, \quad v_{\alpha q(i)}^{+}=v_{\alpha q}^{+} .
$$

The corresponding relations for inverse 11D harmonics read

$$
v_{q(i)}^{+\alpha}=v_{q}^{+\alpha}, \quad v_{q(i)}^{-\alpha}=v_{q}^{-\alpha}-\frac{1}{2} K_{(i)}^{=I} \gamma_{q p}^{I} v_{p}^{+\alpha},
$$

so that eqs. (7.22) and (7.23) drastically simplify

$$
\begin{aligned}
& v_{p(i)}^{+\alpha} v_{\alpha q(j)}^{-}=\delta_{p q}, \\
& v_{p(i)}^{-\alpha} v_{\alpha q(j)}^{-}=\frac{1}{2} K_{j i}^{=I} \gamma_{p q}^{I}, \quad K_{j i}^{=I}:=K_{j}^{=I}-K_{i}^{=I} .
\end{aligned}
$$

The frame vectors are decomposed in the basis provided by the auxiliary frame as

$$
\begin{aligned}
& u_{a(i)}^{\overline{=}}=u_{a}^{=}+K_{(i)}^{=I} u_{a}^{I}+\frac{1}{4}\left(\vec{K}_{(i)}^{\bar{E}}\right)^{2} u_{a}^{\#}, \\
& u_{a(i)}^{I}=u_{a}^{I}+\frac{1}{2} K_{(i)}^{=I} u_{a}^{\#}, \\
& u_{a(i)}^{\#}=u_{a}^{\#} .
\end{aligned}
$$

These expressions for vector harmonics apply for both 10D and 11D cases, while the (7.41) and (7.42) are written for $\mathrm{D}=11$.

Although the $\mathrm{D}=10$ relations can be easily restored from (7.41) and (7.42), for possible reader convenience we write them explicitly:

$$
\begin{aligned}
v_{\alpha q(i)}^{-} & =v_{\alpha q}^{-}+\frac{1}{2} K_{i}^{=I} \gamma_{q \dot{p}}^{I} v_{\alpha \dot{p}}^{+}, & v_{\alpha \dot{q}(i)}^{+} & =v_{\alpha \dot{q}}^{+}, \\
v_{q(i)}^{+\alpha} & =v_{q}^{+\alpha}, & v_{\dot{q}(i)}^{-\alpha} & =v_{\dot{q}}^{-\alpha}-\frac{1}{2} K_{(i)}^{=I} v_{q}^{+\alpha} \gamma_{q \dot{q}}^{I} .
\end{aligned}
$$

Let us apply the above gauge fixing expressions to study the momentum conservation conditions.

\subsection{Momentum conservation and Mandelstam variables}

In our formalism the momentum conservation in the scattering of $n$ massless particles implies

$$
\sum_{i=1}^{n} \rho_{(i)}^{\#} u_{a(i)}^{=}=0 .
$$

Contracting this equation with the basic vectors of the reference frame we can split it into three sets which look especially simple in the gauge (7.40),

$$
\begin{aligned}
\sum_{i=1}^{n} \rho_{(i)}^{\#} & =0, \\
\sum_{i=1}^{n} \rho_{(i)}^{\#} K_{(i)}^{=I} & =0, \\
\sum_{i=1}^{n} \rho_{(i)}^{\#}\left(K_{(i)}^{=I}\right)^{2} & =0 .
\end{aligned}
$$


In the case of 4-point amplitude we find that the expressions for the Mandelstam variables are sufficiently simple already with the generic parametrization of the frame variables (7.28)-(7.30). Keeping all the gauge symmetry unfixed, we obtain from (7.28) and $(7.17)$

$$
\begin{aligned}
s & =\left(k_{1}+k_{2}\right)^{2}=2 \rho_{1}^{\#} \rho_{2}^{\#} u_{2}^{=a} u_{a 1}^{=}=\rho_{1}^{\#} \rho_{2}^{\#} e^{-2 \beta_{21}}\left(\vec{K}_{21}^{\bar{E}}\right)^{2}, \\
t & =\left(k_{1}+k_{3}\right)^{2}=2 \rho_{1}^{\#} \rho_{3}^{\#} u_{3}^{=a} u_{a 1}^{\overline{=}}=\rho_{1}^{\#} \rho_{3}^{\#} e^{-2 \beta_{31}}\left(\vec{K}_{31}^{\overline{ }}\right)^{2}, \\
u & =\left(k_{1}+k_{4}\right)^{2}=2 \rho_{1}^{\#} \rho_{4}^{\#} u_{4}^{=a} u_{a 1}^{=}=\rho_{1}^{\#} \rho_{4}^{\#} e^{-2 \beta_{41}}\left(\vec{K}_{41}^{\overline{ }}\right)^{2} .
\end{aligned}
$$

Notice that the denominators in the first and in the second terms of the BCFW-type relations for the four point amplitudes and superamplitudes, (7.11) and (7.2), are equal to Mandelstam variable $s$ and $t$, respectively

$$
\begin{aligned}
& P_{12}^{2}=s=2 \rho_{1}^{\#} \rho_{2}^{\#} u_{2}^{=a} u_{a 1}^{=}=2 e^{-\beta_{21}-\beta_{43}} \sqrt{\rho_{1}^{\#} \rho_{2}^{\#} \rho_{3}^{\#} \rho_{4}^{\#}} \sqrt{\left(\vec{K}_{\overline{21}}^{\bar{E}}\right)^{2}\left(\vec{K}_{\overline{43}}^{\bar{E}}\right)^{2}} \\
& P_{13}^{2}=t=2 \rho_{1}^{\#} \rho_{2}^{\#} u_{3}^{=a} u_{a(1)}^{=} .
\end{aligned}
$$

In the second equality of (7.57) we have used the conservation of the momentum which implies $s=P_{12}^{2}=P_{43}^{2}=\sqrt{P_{12}^{2} P_{43}^{2}}$.

The set of the arguments of the (super)amplitudes in the r.h.s.'s of (7.11) and (7.2) include (the variables related to) the deformed versions of (7.57) and (7.58), $P_{12}^{a}\left(z_{12}\right)$ and $P_{13}^{a}\left(z_{13}\right)$, which are dependent on the complex null-vector $q_{a}$ obeying (6.37). Let us discuss the representation of this and of the special values $z_{12}, z_{13}$ of the deformation parameter in our spinor frame approach.

\subsection{Studying the BCFW-like deformation with the gauge fixed spinor frames}

In the case of 4-particle amplitude, the deformation 11 -vector/10-vector $q_{a}$, a complex nullvector orthogonal to the 1 -st and the 4 -th light-like momenta, (6.37), can be decomposed on the frame related to any of four light-like momenta (7.17). The decompositions on 1-st and 4-th frames cannot contain terms with $u_{a(1)}^{\#}$ and $u_{a(4)}^{\#}$, respectively. Generically we can also assume the absence of the terms proportional to $u_{a(1)}^{=}$and $u_{a(4)}^{=}$(this is to say, to $k_{a(1)}$ and $\left.k_{a(4)}\right)$ so that

$$
q_{a}=-u_{a(1)}^{I} q_{(1)}^{I}=-u_{a(4)}^{I} q_{(4)}^{I}, \quad\left(\vec{q}_{(1)}\right)^{2}=q_{(1)}^{I} q_{(1)}^{I}=0, \quad\left(\vec{q}_{(4)}\right)^{2}=q_{(4)}^{I} q_{(4)}^{I}=0 .
$$

In the gauge (7.31), in which the relation between vectors from different frames are described by eqs. $(7.32)-(7.34)$, the components $q_{(4)}^{I}$ and $q_{(1)}^{I}$ are related by $\mathrm{SO}(D-2)$ rotation

$$
q_{(4)}^{I}=\mathcal{O}_{41}^{J I} q_{(1)}^{I}
$$

and obey, besides the null-conditions (7.59),

$$
K_{41}^{=I} q_{(1)}^{I}=0, \quad K_{14}^{=I} q_{(4)}^{I}=0 .
$$


Now, one can easily check that the special values $z_{12}$ and $z_{13}$ of the deformation parameter $z$, for which $\widehat{P_{12}^{a}}(z)$ and $\widehat{P_{13}^{a}}(z)$ become light-like, read (see (6.69))

$$
\begin{aligned}
& z_{12}=-\frac{\rho_{(1)}^{\#}\left(u_{(1)}^{\overline{=}} u_{(2)}^{\overline{=}}\right)}{q_{(1)}^{I}\left(u_{(1)}^{I} u_{(2)}^{\bar{E}}\right)}=\frac{\rho_{(1)}^{\#}\left(\vec{K}_{(21)}^{\overline{=}}\right)^{2}}{2 K_{(21)}^{=I} q_{(1)}^{I}} \quad \Rightarrow \quad\left(\widehat{P_{12}}\left(z_{12}\right)\right)^{2}=0,
\end{aligned}
$$

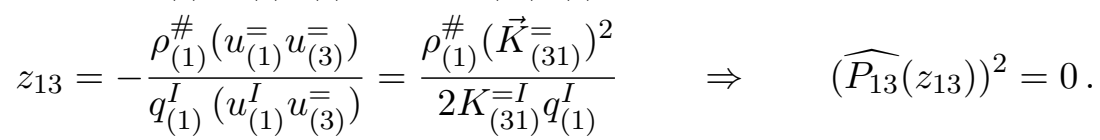

Using (7.59), the nilpotent matrix (6.38) can be written as

$$
\begin{aligned}
\mathbb{M}_{q p} & =-\frac{1}{\sqrt{\rho_{(1)}^{\#} \rho_{(n)}^{\#}}\left(u_{(1)}^{\overline{(})} u_{(n)}^{\bar{y}}\right)}\left(v_{p^{\prime}(1)}^{-\alpha} v_{\alpha q(n)}^{-}\right) \gamma_{p^{\prime} p}^{I} q_{(1)}^{I}= \\
& =-\frac{1}{\sqrt{\rho_{(1)}^{\#} \rho_{(n)}^{\#}}\left(u_{(1)}^{\bar{E}} u_{(n)}^{=}\right)} q_{(n)}^{I} \gamma_{q q^{\prime}}^{I}\left(v_{q^{\prime}(n)}^{-\alpha} v_{\alpha p(1)}^{-}\right) .
\end{aligned}
$$

Furthermore, eq. (7.59) considered together with (7.28) and (6.43), implies

$$
\widehat{u_{(1)}^{=a}}=u_{(1)}^{=a}+u_{(1)}^{I a} \frac{z q_{(1)}^{I}}{\rho_{(1)}^{\#}}, \quad \widehat{u_{(n)}^{=a}}=u_{(n)}^{=a}-u_{(n)}^{I a} \frac{z q_{(n)}^{I}}{\rho_{(n)}^{\#}}
$$

which can be recognized as $S^{9}$-transformations (see (4.14)) with nilpotent 9-vector parameters

$$
\widehat{\mathcal{K}}_{(1)}^{=I}(z)=\frac{z q_{(1)}^{I}}{\rho_{(1)}^{\#}}, \quad \widehat{\mathcal{K}}_{(n)}^{=I}(z)=-\frac{z q_{(n)}^{I}}{\rho_{(n)}^{\#}}
$$

(here we use the notation $\widehat{\mathcal{K}}_{(1)}^{=I}(z)$ reserving $\widehat{K}_{(1)}^{=I}(z)$ for the parameter of transformation relating the deformed 1-st frame with the auxiliary reference frame).

First of all, this observation allows to conclude that $u_{(1)}^{\# a}$ and $u_{(n)}^{\# a}$ vectors may be taken to be undeformed and, using (7.30), to find the deformation of the remaining frame vectors

$$
\widehat{u_{(n)}^{I a}}=u_{(n)}^{I a}-\frac{z q_{(n)}^{I}}{2 \rho_{(n)}^{\#}} u_{(n)}^{\# a}, \quad \widehat{u_{(1)}^{I a}}=u_{(1)}^{I a}+\frac{z q_{(1)}^{I}}{2 \rho_{(1)}^{\#}} u_{(1)}^{\# a} .
$$

Secondly, this implies that the deformation of the spinor frame variables, (6.34) and (6.35), can be written in the form

$$
\begin{aligned}
& \widehat{v_{\alpha q(n)}^{-}}=v_{\alpha q(n)}^{-}+z \sqrt{\frac{\rho_{(1)}^{\#}}{\rho_{(n)}^{\#}}} v_{\alpha p(1)}^{-} \mathbb{M}_{p q}=v_{\alpha q(n)}^{-}-\frac{z}{2 \rho_{(n)}^{\#}} q_{(n)}^{I} \gamma_{q p}^{I} v_{\alpha p(n)}^{+}, \\
& \widehat{v_{\alpha q(1)}^{-}}=v_{\alpha q(1)}^{-}-z \sqrt{\frac{\rho_{(n)}^{\#}}{\rho_{(1)}^{\#}}} \mathbb{M}_{q p} v_{\alpha p(n)}^{-}=v_{\alpha q(1)}^{-}+\frac{z}{2 \rho_{(1)}^{\#}} q_{(1)}^{I} \gamma_{q p}^{I} v_{\alpha p(1)}^{+} .
\end{aligned}
$$


At $z=z_{12}$ and $z=z_{13}$ of $(7.62)$ and $(7.63)$, the momenta $\widehat{P_{12}^{a}}(z)$ and $\widehat{P_{13}^{a}}(z)$ become light-like and can be expressed in terms of 'energies' and the associated spinor frame variables by

$$
\widehat{P_{12}^{a}}\left(z_{12}\right) \Gamma_{a \alpha \beta}=2 \widehat{\rho}^{\#}\left(z_{12}\right) v_{\alpha q(12)}^{-} v_{\beta q(12)}^{-}, \quad \widehat{\rho}^{\#}\left(z_{12}\right) v_{q 12}^{-} \tilde{\Gamma}^{a} v_{p 12}^{-}=\widehat{P_{12}^{a}}\left(z_{12}\right) \delta_{q p}
$$

and its $12 \mapsto{ }_{13}$ counterparts. Factorizing $\widehat{P_{12}}\left(z_{12}\right):=\widehat{P_{12}^{a}}\left(z_{12}\right) \Gamma_{a}=\widehat{\not \phi_{1}}\left(z_{12}\right)+\not k_{2}=\not \phi_{1}+\not k_{2}-$ $z_{12} \phi$, one finds

$$
v_{\alpha q(12)}^{-}=e^{-\beta_{(12) 1}}\left(v_{\alpha q(1)}^{-}+\frac{1}{2} K_{(12) 1}^{=I} \gamma_{q p}^{I} v_{\alpha p(1)}^{+}\right),
$$

with

$$
\begin{aligned}
e^{-\beta_{(12) 1}} & =\sqrt{\left|\frac{\rho_{1}^{\#}+e^{-2 \beta_{21}} \rho_{2}^{\#}}{\widehat{\rho}^{\#}\left(z_{12}\right)}\right|}, \\
K_{(12) 1}^{=I} & =\frac{e^{-2 \beta_{21}} \rho_{2}^{\#}}{\rho_{1}^{\#}+e^{-2 \beta_{21}} \rho_{2}^{\#}} \widehat{K}_{21}^{=I}\left(z_{12}\right), \quad \widehat{K}_{21}^{=I}\left(z_{12}\right)=K_{2}^{=I}-\widehat{K}_{1}^{=I}\left(z_{12}\right)=K_{21}^{=I}-z_{12} q^{I} .
\end{aligned}
$$

Notice that to describe the deformed frame it is convenient to relax a bit the gauge (7.40) allowing for $e^{-2 \beta_{(12) 1}} \neq 1$. Eqs. (7.71) implies

$$
\begin{aligned}
& u_{a(12)}^{=}\left(z_{12}\right)=e^{-2 \beta_{(12) 1}}\left(u_{a(1)}^{=}+\frac{1}{4}\left(\vec{K}_{(12) 1}^{=}\right)^{2} u_{a(1)}^{\#}+K_{(12) 1}^{=I} u_{a(1)}^{I}\right)=\frac{\widehat{P}_{12}\left(z_{12}\right)}{\rho^{\#}\left(z_{12}\right)}, \\
& u_{a(12)}^{\#}\left(z_{12}\right)=e^{+2 \beta_{(12) 1}} u_{a(1)}^{\#}, \\
& u_{a(12)}^{I}\left(z_{12}\right)=u_{a(1)}^{I}+\frac{1}{2} u_{a(1)}^{\#} K_{(12) 1}^{=I} .
\end{aligned}
$$

\subsection{3- and 4- particle kinematics. Momentum conservation}

The momentum conservation condition for our four point amplitudes reads

$$
\rho_{1}^{\#} u_{(1)}^{=a}+\rho_{2}^{\#} u_{(2)}^{=a}+\rho_{3}^{\#} u_{(3)}^{=a}+\rho_{4}^{\#} u_{(4)}^{=a}=0
$$

Generically, this can be split into

$$
\begin{array}{r}
\rho_{1}^{\#}+\tilde{\rho}_{2}^{\# 1}+\tilde{\rho}_{3}^{\# 1}+\tilde{\rho}_{4}^{\# 1}=0, \\
\tilde{\rho}_{2}^{\# 1}\left(\vec{K}_{21}^{\overline{ }}\right)^{2}+\tilde{\rho}_{3}^{\# 1}\left(\vec{K}_{31}^{=}\right)^{2}+\tilde{\rho}_{4}^{\# 1}\left(\vec{K}_{41}^{=}\right)^{2}=0, \\
\tilde{\rho}_{2}^{\# 1} K_{21}^{=I}+\tilde{\rho}_{3}^{\# 1} K_{31}^{=I}+\tilde{\rho}_{4}^{\# 1} K_{41}^{=I}=0
\end{array}
$$

where we have used the notation

$$
\tilde{\rho}_{j}^{\# i}:=e^{-2 \beta_{j i}} \rho_{j}^{\#} .
$$

The three particle kinematics is more restrictive and a nontrivial solution of the momentum conservation conditions, (7.50) with $n=3$, exists for deformed, complexified 
momenta only. Let us write this for the case of BCFW-deformed first momentum and using the notation $P_{12}^{a}\left(z_{12}\right)=\rho^{\#}\left(z_{12}\right) u^{=a}\left(z_{12}\right)$ for the third momentum:

$$
\rho_{1}^{\#} \widehat{u_{1}^{=a}}+\rho_{2}^{\#} u_{2}^{=a}+\rho^{\#}\left(z_{12}\right) u^{=a}\left(z_{12}\right)=0 .
$$

In the gauge (7.40) all the three frames are related with the reference frame by $(7.45),(7.46),(7.47)$ with complex vector parameters

$$
\widehat{K}_{(1)}^{=I}:=\widehat{K}_{(1)}^{=I}\left(z_{12}\right)=K_{(1)}^{=I}+\frac{z_{12} q_{(1)}^{I}}{\rho_{1}^{\#}}=K_{(1)}^{=I}+q_{(1)}^{I} \frac{\left(\vec{K}_{\overline{(2)}}-\vec{K}_{(1)}\right)^{2}}{2 \vec{q}_{(1)}\left(\vec{K}_{(2)}-\vec{K}_{(1)}\right)}
$$

and $K_{(12)}^{=I}\left(z_{12}\right)$ which we are going to find now. This is to say

$$
\begin{gathered}
\widehat{u}_{a 1}^{=}=u_{a}^{=}+\widehat{K}_{1}^{=I} u_{a}^{I}+\frac{1}{4}\left(\widehat{\vec{K}}_{1}^{\overline{ }}\right)^{2} u_{a}^{\#}, \\
u_{a 2}^{=}=u_{a}^{=}+K_{2}^{=I} u_{a}^{I}+\frac{1}{4}\left(\vec{K}_{2}^{=}\right)^{2} u_{a}^{\#}, \\
u_{a(12)}^{=}\left(z_{12}\right)=u_{a}^{=}+K_{(12)}^{=I}\left(z_{12}\right) u_{a}^{I}+\frac{1}{4}\left(\vec{K}_{(12)}^{\overline{=}}\left(z_{12}\right)\right)^{2} u_{a}^{\#} .
\end{gathered}
$$

Substituting these expressions into eq. (7.82), one splits this into the set of three equations (cf. (7.51), (7.52) and (7.53))

$$
\begin{aligned}
& \rho^{\#}\left(z_{12}\right)+\rho_{1}^{\#}+\rho_{2}^{\#}=0, \\
& \rho^{\#}\left(z_{12}\right) K_{(12)}^{=I}\left(z_{12}\right)+\rho_{1}^{\#} \widehat{K}_{1}^{=I}+\rho_{2}^{\#} K_{2}^{=I}=0, \\
& \rho^{\#}\left(z_{12}\right)\left(\vec{K}_{(12)}^{=I}\left(z_{12}\right)\right)^{2}+\rho_{1}^{\#}\left(\widehat{\vec{K}}_{1}^{=I}\right)^{2}+\rho_{2}^{\#}\left(\vec{K}_{2}^{=I}\right)^{2}=0 \text {, }
\end{aligned}
$$

which imply

$$
\begin{aligned}
\rho^{\#}\left(z_{12}\right) & =-\rho_{1}^{\#}-\rho_{2}^{\#}, \\
K_{(12)}^{=I}\left(z_{12}\right) & =\frac{\rho_{1}^{\#}}{\rho_{1}^{\#}+\rho_{2}^{\#}} \widehat{K}_{1}^{=I}+\frac{\rho_{2}^{\#}}{\rho_{1}^{\#}+\rho_{2}^{\#}} K_{2}^{=I},
\end{aligned}
$$

and

$$
\left(\widehat{\vec{K}}_{12}^{=}\right)^{2}=0, \quad \widehat{K}_{21}^{=I}=\left(K_{2}^{=I}-\widehat{K}_{1}^{=I}\right)
$$

This latter however is satisfied identically due to $\vec{q}_{(1)}^{2} \equiv q_{(1)}^{I} q_{(1)}^{I}=0$ (see (7.83)).

For the application it is convenient to write (7.91) also in the form

$$
\begin{aligned}
& K_{(12) 1}^{=I}\left(z_{12}\right):=K_{(12)}^{=I}\left(z_{12}\right)-\widehat{K}_{1}^{=I}=\frac{\rho_{2}^{\#}}{\rho_{1}^{\#}+\rho_{2}^{\#}} \widehat{K}_{21}^{=I}, \\
& K_{(12) 2}^{=I}\left(z_{12}\right):=K_{(12)}^{=I}\left(z_{12}\right)-K_{2}^{=I}=-\frac{\rho_{1}^{\#}}{\rho_{1}^{\#}+\rho_{2}^{\#}} \widehat{K}_{21}^{=I} .
\end{aligned}
$$

Then (7.92) implies nilpotency of $K_{(12) 1}^{=I}\left(z_{12}\right)$ and $K_{(12) 2}^{=I}\left(z_{12}\right)$ :

$$
\left(K_{(12) 1}^{=I}\left(z_{12}\right)\right)^{2}=0, \quad\left(K_{(12) 2}^{=I}\left(z_{12}\right)\right)^{2}=0 .
$$




\section{$7.8 t_{3}$ tensor}

Now we are ready to calculate the expression for $t_{3}$ tensor $(7.13)$ with arguments $\widehat{k_{a(1)}}$, $k_{a(2)}$ and $-\widehat{k_{a(1)}}-k_{a(2)}$, a square of which determines, through (7.12), the first partial amplitude,

$$
\mathcal{A}_{z_{12}\left(I_{1} J_{1}\right)\left(I_{2} J_{2}\right)(I J)}=\mathcal{A}_{z_{12}\left(I_{1} J_{1}\right)\left(I_{2} J_{2}\right)(I J)}\left(\widehat{k_{1}}, k_{2} ; \widehat{\rho} \widehat{\rho}^{\#}\left(z_{12}\right), v_{q}^{-}\left(z_{12}\right)\right)
$$

in the r.h.s. of (7.11). The gauge invariant expression is not simple

$$
\begin{aligned}
& t^{I_{1} I_{2} I_{3}}\left(\widehat{k_{(1)}}, k_{(2)}, \widehat{k_{(1)}}-k_{(2)}\right)= \\
& \quad-\rho_{(1)}^{\#}\left(u_{(1)}^{=} u_{(2)}^{I_{2}}\right) \delta^{I_{1} I_{3}}-z_{12} q_{(1)}^{J}\left(u_{(1)}^{J} u_{(2)}^{I_{2}}\right) \delta^{I_{1} I_{3}}-z_{12}\left(u_{(1)}^{I_{1}} u_{(2)}^{I_{2}}\right) q_{(1)}^{I_{3}} \\
& \quad+\rho_{(2)}^{\#}\left(u_{(1)}^{I_{1}} u_{(2)}^{\bar{E}}\right)\left(u_{(2)}^{I_{2}} u_{(1)}^{I_{3}}\right)+\rho_{(1)}^{\#} K_{(12) 1}^{=I_{3}}\left(u_{(1)}^{I_{1}} u_{(2)}^{I_{2}}\right)+\frac{\rho_{(2)}^{\#}}{2} K_{(12) 1}^{=I_{1}}\left(u_{(1)}^{I_{3}} u_{(2)}^{I_{2}}\right)\left(u_{(1)}^{\#} u_{(2)}\right) \\
& \quad+\frac{z_{12}}{2} q_{(1)}^{I_{1}}\left(u_{(1)}^{\#} u_{(2)}^{I_{2}}\right) K_{(12) 1}^{=I_{3}}-\frac{\left(z_{12}\right)^{2}}{\rho_{(1)}^{\#}} q_{(1)}^{I_{1}}\left(u_{(1)}^{\#} u_{(2)}^{I_{2}}\right) q_{(1)}^{I_{3}} .
\end{aligned}
$$

However, in the gauge (7.40), and after the use of the momentum conservation conditions (7.90) and (7.91), this simplifies essentially:

$$
\begin{aligned}
& t^{I_{1} I_{2} I_{3}}\left(\widehat{k_{(1)}}\left(z_{12}\right), k_{(2)},-\widehat{k_{(1)}}\left(z_{12}\right)-k_{(2)}\right)= \\
& \quad=\delta^{I_{2} I_{3}} \rho_{(2)}^{\#} \widehat{K}_{21}^{=I_{1}}+\rho_{(1)}^{\#} \widehat{K}_{21}^{=I_{2}} \delta^{I_{1} I_{3}}+\delta^{I_{1} I_{2}} \frac{\rho_{(1)}^{\#} \rho_{(2)}^{\#}}{\rho_{(1)}^{\#}+\rho_{(2)}^{\#}} \widehat{K}_{21}^{=I_{3}} .
\end{aligned}
$$

Here the complex $\mathrm{SO}(9)$ vector

$$
\begin{aligned}
\widehat{K}_{21}^{=I}:=\widehat{K}_{21}^{=I}\left(z_{12}\right) & =K_{2}^{=I}-\widehat{K}_{1}^{=I}= \\
& =K_{2}^{=I}-K_{1}^{=I}-q_{(1)}^{I} \frac{\left(\vec{K}_{(2)}^{\overline{(2)}}-\vec{K}_{(1)}\right)^{2}}{2 \vec{q}_{(1)}\left(\vec{K}_{(2)}^{\overline{(2)}}-\vec{K}_{(1)}\right)}
\end{aligned}
$$

is null (7.92) because its square is proportional to $\vec{q}_{(1)}^{2}=0$.

\subsection{Supermomentum}

In construction of superamplitudes one might want to use a sum of $\theta_{q(i)}^{-}$with different $i$-th. The construction of this is hampered by the fact that $\theta_{q(i)}^{-}$'s with different values of $i$ are transformed by different $\left[\mathrm{SO}(1,1)_{i} \otimes \mathrm{SO}(D-2)_{i}\right]$ symmetry groups.

The $\prod_{i=1}^{n}\left[\mathrm{SO}(1,1)_{i} \otimes \mathrm{SO}(D-2)_{i}\right]$ invariant sum of all $\theta_{q(i)}^{-}$does exist but carries a $\operatorname{Spin}(1, D-1)$ index $\alpha$ with twice more values (32 in $\mathrm{D}=11)$ than $\operatorname{Spin}(D-2)$ index $q(16$ in $\mathrm{D}=11$ ),

$$
\mathfrak{q}_{\alpha}:=\sum_{i=1}^{n} \rho_{(i)}^{\#} v_{\alpha q(i)}^{-} \theta_{p(i)}^{-} .
$$


Following the custom of $\mathrm{D}=4$ amplitude literature we call this 'supermomentum'; it is superpartner of the total momentum $\sum_{i=1}^{n} p_{a(i)}$ and hence is supersymmetric invariant as far as the total momentum is conserved, $\sum_{i=1}^{n} p_{a(i)}=0$ :

$$
\delta_{\epsilon} \mathfrak{q}_{\alpha}=\epsilon^{\beta} \sum_{i=1}^{n} \rho_{(i)}^{\#} v_{\alpha q(i)}^{-} v_{\beta q(i)}^{-}=\frac{1}{2} \Gamma_{\alpha \beta}^{a} \epsilon^{\beta} \sum_{i=1}^{n} p_{a(i)}=0 .
$$

The supersymmetry invariance is equivalent to stating that, when $\sum_{i=1}^{n} p_{a(i)}=0$, the supermomentum can be equivalently written in the form

$$
\mathfrak{q}_{\alpha}:=\sum_{i=1}^{n} \rho_{(i)}^{\#} v_{\alpha q(i)}^{-}\left(\theta_{p(i)}^{-}-\Xi^{\beta} v_{\beta q(i)}^{-}\right)
$$

with an arbitrary fermionic spinor $\Xi^{\beta}$. This spinor can identified with global supersymmetry parameter and the above equation makes transparent that this can be used to cancel the contribution of one of the fermionic variable, say $\theta_{p(1)}^{-}$. Actually, as we will see in no time, the supermomentum depends on $(n-2)$ linear combinations of $n$ fermionic variables.

In the gauge (7.40) we can obtain a simple decomposition of supermomentum on the auxiliary reference spinor frame,

$$
\mathfrak{q}_{\alpha}=v_{\alpha q}^{-} \sum_{i=1}^{n} \rho_{(i)}^{\#} \theta_{q(i)}^{-}+\frac{1}{2} v_{\alpha p}^{+} \gamma_{p q}^{I} \sum_{i=1}^{n} \rho_{(i)}^{\#} K_{(i)}^{=I} \theta_{q(i)}^{-} .
$$

We can split this in a Lorentz covariant manner on

$$
\begin{aligned}
& \mathfrak{q}_{q}^{+}=v_{q}^{+\alpha} \mathfrak{q}_{\alpha}=\sum_{i=1}^{n} \rho_{(i)}^{\#} \theta_{q(i)}^{-}=\sum_{i=2}^{n} \rho_{(i)}^{\#}\left(\theta_{q(i)}^{-}-\theta_{q(1)}^{-}\right) \\
& \mathfrak{q}_{q}^{-}=v_{q}^{-\alpha} \mathfrak{q}_{\alpha}=\frac{1}{2} \gamma_{q p}^{I} \sum_{i=1}^{n} \rho_{(i)}^{\#} K_{(i)}^{=I} \theta_{p(i)}^{-}=\frac{1}{2} \gamma_{q p}^{I} \sum_{i=2}^{n} \rho_{(i)}^{\#} K_{(i)}^{=I}\left(\theta_{p(i)}^{-}-\theta_{p(1)}^{-}\right)
\end{aligned}
$$

where the last parts of equalities are obtained by using the momentum conservation conditions (cf. (7.101)).

We can also use one of the spinor frame associated to a scattering particle instead of the reference spinor frame thus obtaining the supermomentum projections

$$
\begin{aligned}
\mathfrak{q}_{q j}^{+}=v_{q j}^{+\alpha} \mathfrak{q}_{\alpha} & =\sum_{i=1, i \neq j}^{n} \rho_{(i)}^{\#} \theta_{q(i)}^{-}=\sum_{i \neq j, 1}^{n} \rho_{(i)}^{\#}\left(\theta_{q(i)}^{-}-\theta_{q(1)}^{-}\right) \\
\mathfrak{q}_{q j}^{-}=v_{q j}^{-\alpha} \mathfrak{q}_{\alpha} & =\frac{1}{2} \gamma_{p q}^{I} \sum_{i=1 ; i \neq j}^{n} \rho_{(i)}^{\#} K_{(i j)}^{=I} \theta_{q(i)}^{-}=\frac{1}{2} \gamma_{q p}^{I} \sum_{i \neq j, 1}^{n} \rho_{(i)}^{\#} K_{(i j)}^{=I}\left(\theta_{p(i)}^{-}-\theta_{p(1)}^{-}\right) .
\end{aligned}
$$

These relations make transparent that actually the supermomentum depends on only $(n-2)$ (linear combinations) of $n$ fermionic variables. Furthermore, the projection $\mathfrak{q}_{q j}^{-}$(7.106) looks more interesting as it contains more information about momenta of scattered particle and also because it is defined with the use of spinor helicity variables only, while $\mathfrak{q}_{q j}^{+}$is defined with the use of $j$-th complementary spinor $v_{q j}^{+\alpha}$ and thus is not covariant under $K_{9(i)}$ symmetry $((4.13)$ with $\dot{q}=q=1, \ldots, 16)$. 


\subsubsection{Invariants from the projections of supermomentum}

Using (7.104) or (7.106) we can introduce the covariant delta functions for integration over 16 fermionic variables

$$
\delta^{16}\left(\mathfrak{q}^{-}\right)=\left(\mathfrak{q}^{-}\right)^{\wedge 16}:=\frac{1}{16 !} \epsilon^{q_{1} \ldots q_{16}} \mathfrak{q}_{q_{1}}^{-} \ldots \mathfrak{q}_{q_{16}}^{-},
$$

which has the weight -16 under auxiliary $\mathrm{SO}(1,1)_{0}$ gauge symmetry.

Notice also the existence of weight $-8 \mathrm{SO}(9)$ invariant

$$
\left(\mathfrak{q}^{-}\right)^{\wedge 8}:=\frac{1}{c_{8}} \epsilon^{I_{1} I_{2} \ldots I_{9}}\left(\mathfrak{q}^{-} \gamma^{I_{1} I_{2} I_{3}} \mathfrak{q}^{-}\right)\left(\mathfrak{q}^{-} \gamma^{I_{4} I_{5}} \mathfrak{q}^{-}\right) \ldots\left(\mathfrak{q}^{-} \gamma^{I_{8} I_{9}} \mathfrak{q}^{-}\right)
$$

In it we prefer to chose the coefficient

$$
c_{8}=\sqrt{\frac{1}{16 !} \epsilon^{I_{1} I_{2} \ldots I_{9}} \epsilon^{J_{1} J_{2} \ldots J_{9}} \epsilon^{q_{1} \ldots q_{16}} \gamma_{q_{1} q_{2}}^{I_{1} I_{2} I_{3}} \gamma_{q_{3} q_{4}}^{I_{4} I_{5}} \ldots \gamma_{q_{7} q_{8}}^{I_{8} I_{9}} \gamma_{q_{9} q_{10}}^{J_{1} J_{2} J_{3}} \gamma_{q_{11} q_{12}}^{J_{4} J_{5}} \ldots \gamma_{q_{15} q_{16}}^{J_{8} J_{9}}}
$$

which makes (7.108) the exact square root of the fermionic delta function

$$
\left(\mathfrak{q}^{-}\right)^{\wedge 8}\left(\mathfrak{q}^{-}\right)^{\wedge 8}=\left(\mathfrak{q}^{-}\right)^{\wedge 16} \equiv \delta^{16}\left(\mathfrak{q}^{-}\right) .
$$

One can also introduce a weight -4 supersymmetric invariant

$$
\left(\mathfrak{q}^{-}\right)^{\wedge 4}=\frac{1}{c_{4}}\left(\mathfrak{q}^{-} \gamma^{I J} \mathfrak{q}^{-}\right)\left(\mathfrak{q}^{-} \gamma^{I J} \mathfrak{q}^{-}\right)
$$

Notice that in $\mathrm{D}=10$ the counterpart of (7.104)

$$
\mathfrak{q}_{\dot{q}}^{-}=v_{\dot{q}}^{-\alpha} \mathfrak{q}_{\alpha}=\frac{1}{2} \gamma_{q \dot{q}}^{I} \sum_{i=1}^{n} \rho_{(i)}^{\#} K_{(i)}^{=I} \theta_{q(i)}^{-}=\frac{1}{2} \gamma_{q \dot{q}}^{I} \sum_{i=2}^{n} \rho_{(i)}^{\#} K_{(i)}^{=I}\left(\theta_{q(i)}^{-}-\theta_{q(1)}^{-}\right),
$$

carries the dotted index $\dot{q}=1, \ldots, 8$ and the counterpart of (7.111) will be playing the role of square root of the $\mathrm{SO}(8)$ invariant fermionic delta function, while the counterpart of (7.108),

$$
D=10: \quad\left(\mathfrak{q}^{-}\right)^{\wedge 8}:=\frac{1}{c_{8}^{\prime}} \epsilon^{I_{1} I_{2} \ldots I_{8}}\left(\mathfrak{q}^{-} \tilde{\gamma}^{I_{1} I_{2}} q^{-}\right)\left(\mathfrak{q}^{-} \tilde{\gamma}^{I_{3} I_{4}} \mathfrak{q}^{-}\right) \ldots\left(\mathfrak{q}^{-} \tilde{\gamma}^{I_{7} I_{8}} \mathfrak{q}^{-}\right)=\delta^{8}\left(\mathfrak{q}^{-}\right)
$$

with an appropriate choice of $c_{8}^{\prime}$, coincides with this fermionic delta function.

\subsubsection{Invariants from the complete spinorial supermomentum}

One can also construct some Lorentz invariant combinations of the complete supermomentum with Lorentz group spinor indices. In $D=10$ the product of two copies of fermionic spinors belongs to $\mathbf{1 2 0}$ antisymmetric tensor representation of the Lorentz group. This is to say $\mathfrak{q}_{\alpha} \mathfrak{q}_{\beta}=\propto(\mathfrak{q})_{a b c}^{\wedge 2} \sigma_{\alpha \beta}^{a b c}$ with

$$
D=10: \quad(\mathfrak{q})_{a b c}^{\wedge 2}:=\mathfrak{q}_{\alpha} \tilde{\sigma}_{a b c}^{\alpha \beta} \mathfrak{q}_{\beta},
$$


so that there exists a unique 4 -th order invariant

$$
D=10: \quad(\mathfrak{q})^{\wedge 4(s)}=(\mathfrak{q})^{\wedge 2 a b c}(\mathfrak{q})_{a b c}^{\wedge 2} .
$$

This is the trace of the forth order second rank tensor

$$
(\mathfrak{q})^{\wedge 4(t)}{ }_{a}^{b}:=(\mathfrak{q})_{a c d}^{\wedge 2}(\mathfrak{q})^{\wedge 2 b c d}
$$

which can be also used to form invariants of $4 m$ degree with $m=2,3,4$,

$$
D=10: \quad(\mathfrak{q})^{\wedge 4 m(s)}=(\mathfrak{q})^{\wedge 4(t)}{ }_{a} b_{1} \ldots(\mathfrak{q})^{\wedge 4(t)}{ }_{b_{m-1}}{ }^{a}=: \operatorname{Tr}\left((\mathfrak{q})^{\wedge 4(t)}\right)^{m}, \quad m=2,3,4
$$

Clearly, $m=4$ invariant is proportional to delta function of the spinorial supermomentum, while $m=2$ invariant can be considered as square root of this latter,

$$
D=10: \quad(\mathfrak{q})^{\wedge 8(s)}(\mathfrak{q})^{\wedge 8(s)} \propto(\mathfrak{q})^{\wedge 16(s)} \propto \delta^{16}\left(\mathfrak{q}_{\alpha}\right) .
$$

In $D=11$ the product of two copies of a fermionic spinor decomposes on three irreducible representations, $\mathbf{3 2} \times \mathbf{3 2}=\mathbf{1}+\mathbf{1 6 5}+\mathbf{3 3 0}$. This is to say, their exists a second order invariant

$$
D=11: \quad(\mathfrak{q})^{\wedge 2(s)}:=\mathfrak{q}_{\alpha} C^{\alpha \beta} \mathfrak{q}_{\beta},
$$

and two second order tensors constructed from the spinorial supermomentum bilinears

$$
D=11: \quad(\mathfrak{q})_{a b c}^{\wedge 2}:=\mathfrak{q}_{\alpha} \tilde{\Gamma}_{a b c}^{\alpha \beta} \mathfrak{q}_{\beta}, \quad(\mathfrak{q})_{a b c d}^{\wedge 2}:=\mathfrak{q}_{\alpha} \tilde{\Gamma}_{a b c d}^{\alpha \beta} \mathfrak{q}_{\beta} .
$$

This can be used to construct invariants of higher order. In particular there exist two 4-th order invariants,

$$
D=10: \quad(\mathfrak{q})^{\wedge 4(s 1)}=(\mathfrak{q})^{\wedge 2 a b c}(\mathfrak{q})_{a b c}^{\wedge 2}, \quad(\mathfrak{q})^{\wedge 4(s 2)}=(\mathfrak{q})^{\wedge 2 a b c d}(\mathfrak{q})_{a b c d}^{\wedge 2}
$$

and the following interesting 6 -th order invariant

$$
D=11: \quad(\mathfrak{q})^{\wedge 6(s)}:=\epsilon^{a_{1} \ldots a_{11}}(\mathfrak{q})_{a_{1} a_{2} a_{3}}^{\wedge 2}(\mathfrak{q})_{a_{4} a_{5} a_{6} a_{7}}^{\wedge 2}(\mathfrak{q})_{a_{8} a_{9} a_{10} a_{11}}^{\wedge 2}
$$

We also have two second rank fourth order tensors, $(\mathfrak{q})^{\wedge 4(t 1)} b_{b}{ }^{a}=(\mathfrak{q})_{b c d}^{\wedge 2}(\mathfrak{q})^{\wedge 2 \text { acd }}$ and $(\mathfrak{q})^{\wedge 4(t 2)} b^{a}=(\mathfrak{q})_{b c d e}^{\wedge 2}(\mathfrak{q})^{\wedge 2 \text { acde }}$ the traces of which give $(7.121)$, so that zoo of the $11 \mathrm{D}$ supersymmetric and Lorentz invariants constructed from supermomentum is even reacher that the 10D one. In particular there exists an interesting (although not unique) 8-order invariant

$$
D=11: \quad(\mathfrak{q})^{\wedge 8(s)}:=\epsilon^{a_{1} \ldots a_{11}}(\mathfrak{q})_{a_{1}}^{\wedge 2 b c}(\mathfrak{q})_{b c a_{2} a_{3}}^{\wedge 2}(\mathfrak{q})_{a_{4} a_{5} a_{6} a_{7}}^{\wedge 2}(\mathfrak{q})_{a_{8} a_{9} a_{10} a_{11}}^{\wedge 2}
$$

and a number of 16-th order invariants, all of which can be treated as square roots of the fermionic delta function $\delta^{32}\left(\mathfrak{q}_{\alpha}\right)$. 


\section{Studying the candidate BCFW-type recurrent relations for 4-particle amplitudes of 10D SYM}

To check consistency and completeness of our BCFW deformation and candidate BCFWtype recurrent relations, in this section we will try to obtain on their basis 4-particle amplitudes of 10D SYM with 4 and 2 fermionic legs. Unfortunately, this calculation indicates a problem: the candidate amplitudes obtained from $\mathrm{D}=10 \mathrm{BCFW}$ procedure suffer an unwanted dependence on the deformation vectors. We nevertheless find useful to describe these calculations as they might suggest a resolution of the issue which is probably general for higher dimensional BCFW-type constructions.

\subsection{Candidate BCFW for 4-fermionic amplitude in 10D SYM from super-BCFW for 4-point superamplitude}

The candidate BCFW relation for the four fermionic superamplitude of 10D SYM is

$$
\begin{aligned}
\mathcal{A}_{\dot{q}_{1} \dot{q}_{2} \dot{q}_{3} \dot{q}_{4}}\left(k_{1}, \theta_{(1)}^{-} ; k_{2}, \theta_{(2)}^{-} ; k_{3}, \theta_{(3)}^{-} ; k_{4}, \theta_{(4)}^{-}\right)= \\
=\frac{1}{16\left(\widehat{\rho}^{\#}\left(z_{12}\right)\right)^{2}}\left(D _ { q ( z _ { 1 2 } ) } ^ { + } \left(\mathcal{A}_{z_{12} \dot{q}_{1} \dot{q}_{2} \dot{p}}\left(\widehat{k_{1}}, \widehat{\theta_{(1)}^{-}} ; k_{2}, \theta_{(2)}^{-} ; \widehat{P_{12}}\left(z_{12}\right), \Theta^{-}\right) \times\right.\right. \\
\left.\left.\quad \times \frac{1}{\left(P_{12}\right)^{2}} \overleftrightarrow{D}_{q\left(z_{12}\right)}^{+} \mathcal{A}_{z_{12} \dot{p} \dot{q}_{3} \dot{q}_{4}}\left(-\widehat{P_{12}}\left(z_{12}\right), \Theta^{-} ; k_{3}, \theta_{(3)}^{-} ; \widehat{k_{4}}, \widehat{\theta_{(4)}^{-}}\right)\right)\right)_{\Theta_{q}^{-}=0} \\
-\frac{1}{16\left(\widehat{\rho}^{\#}\left(z_{13}\right)\right)^{2}}\left(D _ { q ( z _ { 1 3 } ) } ^ { + } \left(\mathcal{A}_{z_{13} \dot{q}_{1} \dot{q}_{3} \dot{p}}\left(\widehat{k_{1}}, \widehat{\theta_{(1)}^{-}} ; k_{3}, \theta_{(3)}^{-} ; \widehat{P_{13}}\left(z_{13}\right), \Theta^{-}\right) \times\right.\right. \\
\left.\left.\quad \times \frac{1}{\left(P_{13}\right)^{2}} \overleftrightarrow{D}_{q\left(z_{13}\right)}^{+} \mathcal{A}_{z_{13} \dot{p} \dot{q}_{2} \dot{q}_{4}}\left(-\widehat{P_{13}}\left(z_{13}\right), \Theta^{-} ; k_{2}, \theta_{(2)}^{-} ; \widehat{k_{4}}, \widehat{\theta_{(4)}^{-}}\right)\right)\right)_{\Theta_{q}^{-}=0}
\end{aligned}
$$

After applying the covariant derivatives, this expression can be written in the form

$$
\begin{aligned}
\mathcal{A}_{\dot{q}_{1} \dot{q}_{2} \dot{q}_{3} \dot{q}_{4}} & \left(k_{1}, \theta_{(1)}^{-} ; k_{2}, \theta_{(2)}^{-} ; k_{3}, \theta_{(3)}^{-} ; k_{4}, \theta_{(4)}^{-}\right)= \\
=- & 2 \mathcal{A}_{z_{12} \dot{q}_{1} \dot{q}_{2} \dot{p}}\left(\widehat{k_{1}}, \widehat{\theta_{(1)}^{-}} ; k_{2}, \theta_{(2)}^{-} ; \widehat{\rho^{\#}}\left(z_{12}\right), v_{q}^{-}\left(z_{12}\right), 0\right) \\
& \times \frac{1}{\left(P_{12}\right)^{2} \widehat{\rho^{\#}}\left(z_{12}\right)} \mathcal{A}_{z_{12}} \dot{p} \dot{q}_{3} \dot{q}_{4}\left(-\widehat{\rho^{\#}}\left(z_{12}\right), v_{q}^{-}\left(z_{12}\right), 0 ; k_{3}, \theta_{(3)}^{-} ; \widehat{k_{4}}, \widehat{\theta_{(4)}^{-}}\right) \\
+ & \mathcal{A}_{z_{12} \dot{q}_{1} \dot{q}_{2} I}\left(\widehat{k_{1}}, \widehat{\theta_{(1)}^{-}} ; k_{2}, \theta_{(2)}^{-} ; \widehat{\rho^{\#}}\left(z_{12}\right), v_{q}^{-}\left(z_{12}\right), 0\right) \\
\quad & \times \frac{1}{\left(P_{12}\right)^{2}} \mathcal{A}_{z_{12} I \dot{q}_{3} \dot{q}_{4}}\left(-\widehat{\rho^{\#}}\left(z_{12}\right), v_{q}^{-}\left(z_{12}\right), 0 ; k_{3}, \theta_{(3)}^{-} ; \widehat{k_{4}}, \widehat{\theta_{(4)}^{-}}\right)-(2 \longleftrightarrow 3) .
\end{aligned}
$$

In the assumption that amplitudes are reproduced as leading components of superamplitudes, the BCFW relation for the 4-fermionic amplitude of 10D SYM reads

$$
\begin{aligned}
\mathcal{A}_{\dot{q}_{1} \dot{q}_{2} \dot{q}_{3} \dot{q}_{4}} & \left(k_{1} ; k_{2} ; k_{3} ; k_{4}\right)= \\
= & \mathcal{A}_{z_{12} \dot{q}_{1} \dot{q}_{2} I}\left(\widehat{k_{1}} ; k_{2}, ; \widehat{\rho^{\#}}\left(z_{12}\right), v_{q}^{-}\left(z_{12}\right)\right) \\
& \times \frac{1}{\left(P_{12}\right)^{2}} \mathcal{A}_{z_{12} I \dot{q}_{3} \dot{q}_{4}}\left(-\widehat{\rho^{\#}}\left(z_{12}\right), v_{q}^{-}\left(z_{12}\right) ; k_{3}, ; \widehat{k_{4}}\right)-(2 \longleftrightarrow 3),
\end{aligned}
$$

where we have taken into account that the amplitudes of the processes with odd number of fermions vanish, in particular $\mathcal{A}_{z_{12} \dot{q}_{1} \dot{q}_{2} \dot{p}}\left(\widehat{k_{1}} ; k_{2}, ; \widehat{\rho^{\#}}\left(z_{12}\right), v_{q}^{-}\left(z_{12}\right)\right) \equiv 0$. 


\subsection{3-point amplitudes with two fermionic particles in 10D SYM}

In the case of 10D SYM the expression for tree 3-point amplitudes with two fermionic legs is suggested by light-cone string vertices of [103]. In our notation it reads

$$
\begin{aligned}
& \mathcal{A}_{\dot{q}_{1} \dot{q}_{2} I}\left(\rho_{(1)}^{\#}, v_{q(1)}^{-} ; \rho_{(2)}^{\#}, v_{q(2)}^{-} ; \rho_{3}^{\#}, v_{q 3}^{-}\right)=\frac{1}{4} \rho_{1}^{\#} \rho_{2}^{\#} v_{\dot{q}_{1}(1)}^{-\alpha} v_{\dot{q}_{2}(2)}^{-\beta} \sigma_{\alpha \beta}^{a} u_{a(3)}^{I} \\
& =\frac{1}{2} \rho_{1}^{\#} \rho_{2}^{\#} \gamma_{p \dot{p}}^{I}\left(v_{\dot{q}_{1}(1)}^{-\alpha} v_{\alpha p(3)}^{-} v_{\dot{q}_{2}(2)}^{-\beta} v_{\beta \dot{p}(3)}^{+}+v_{\dot{q}_{1}(1)}^{-\alpha} v_{\alpha \dot{p}(3)}^{+} v_{\dot{q}_{2}(2)}^{-\beta} v_{\beta p(3)}^{-}\right) .
\end{aligned}
$$

In distinction to [103], here we assume that $\rho_{i}^{\#}, v_{\dot{q}_{i}(i)}^{-\alpha}$ obey the momentum conservation; so that the spinor frame variables should be complex/deformed. The form of the amplitude, the multipliers and coefficients in (8.3) can be checked by Ward identities.

Indeed, in the gauge (7.40) the expression (8.3) simplifies to

$$
\mathcal{A}_{\dot{q}_{1} \dot{q}_{2} I}\left(\rho_{(1)}^{\#}, v_{q(1)}^{-} ; \rho_{(2)}^{\#}, v_{q(2)}^{-} ; \rho_{(3)}^{\#}, v_{q 3}^{-}\right)=\frac{1}{4} \rho_{1}^{\#} \rho_{2}^{\#} \gamma_{p \dot{p}}^{I}\left(K_{31}^{=J} \gamma_{p \dot{q}_{1}}^{J} \delta_{\dot{q}_{2} \dot{p}}+K_{32}^{=J} \gamma_{p \dot{q}_{2}}^{J} \delta_{\dot{q}_{1} \dot{p}}\right)
$$

and it is straightforward to check that this expression obeys the Ward identity (4.32). Furhtermore, it obeys (4.33) which also fixes the form of purely bosonic 3-point amplitude to be $\mathrm{be}^{19}$

$$
\mathcal{A}_{I_{1} I_{2} I_{3}}=-\left(\delta^{I_{1} I_{2}} \rho_{1}^{\#} K_{13}^{=I_{3}}+\delta^{I_{2} I_{3}} \rho_{2}^{\#} K_{21}^{=I_{1}}+\delta^{I_{3} I_{1}} \rho_{3}^{\#} K_{32}^{=I_{2}}\right)=-t^{I_{1} I_{2} I_{3}}
$$

The last part of (8.5) is given by the projection of the $t$-tensor [103], (7.13).

In (7.13) $u_{a(i)}^{I_{i}}$ are considered to be polarization vectors of $i$-th particle and the momenta are assumed to be proportional to the light-like vectors $u_{a(i)}^{=}$from the same frame (4.2), $k_{a i}=\rho_{i}^{\#} u_{a i}^{=}$. Then in the gauge (7.41), where (7.45), (7.46), (7.47) hold, one finds the gauge fixed form of $t^{I_{1} I_{2} I_{3}}$ indicated in (8.5).

\subsection{Testing the candidate BCFW by calculating 4-gluino amplitude. Problem of dependence on deformation vector}

As a test of our candidate BCFW relation (8.2), let us use it to calculate the gauge fixed expression for the tree 4-fermion amplitudes of 10D SYM. Substituting (8.4), one finds

$$
\begin{aligned}
& \mathcal{A}_{\dot{q}_{1} \dot{q}_{2} \dot{q}_{3} \dot{q}_{4}}\left(k_{1} ; k_{2} ; k_{3} ; k_{4}\right)=\frac{\sqrt{\rho_{1}^{\#} \rho_{2}^{\#} \rho_{3}^{\#} \rho_{4}^{\#}}}{8} \\
& \times\left(\frac{\widehat{K_{21}^{=J}}\left(z_{12}\right) \widehat{K_{43}^{=K}}\left(z_{12}\right)}{\left.\sqrt{\left(\vec{K}_{21}^{\bar{I}}\right)^{2}\left(\vec{K}_{43}=\right.}\right)^{2}}\left(\left(\tilde{\gamma}^{J} \gamma^{I}\right)_{\dot{q}_{1} \dot{q}_{2}}-\frac{2 \rho_{1}^{\#}}{\rho_{1}^{\#}+\rho_{2}^{\#}} \delta^{J I} \delta_{\dot{q}_{1} \dot{q}_{2}}\right)\left(\left(\tilde{\gamma}^{K} \gamma^{I}\right)_{\dot{q}_{3} \dot{q}_{4}}-\frac{2 \rho_{4}^{\#}}{\rho_{3}^{\#}+\rho_{4}^{\#}} \delta^{K I} \delta_{\dot{q}_{3} \dot{q}_{4}}\right)\right. \\
& \left.-\frac{\widehat{K_{31}^{=J}}\left(z_{13}\right) \widehat{K_{42}^{=K}}\left(z_{13}\right)}{\sqrt{\left(\vec{K}_{31}^{\bar{E}}\right)^{2}\left(\vec{K}_{42}^{\overline{ }}\right)^{2}}}\left(\left(\tilde{\gamma}^{J} \gamma^{I}\right)_{\dot{q}_{1} \dot{q}_{3}}-\frac{2 \rho_{1}^{\#}}{\rho_{1}^{\#}+\rho_{3}^{\#}} \delta^{J I} \delta_{\dot{q}_{1} \dot{q}_{3}}\right)\left(\left(\tilde{\gamma}^{K} \gamma^{I}\right)_{\dot{q}_{2} \dot{q}_{4}}-\frac{2 \rho_{4}^{\#}}{\rho_{2}^{\#}+\rho_{4}^{\#}} \delta^{K I} \delta_{\dot{q}_{2} \dot{q}_{4}}\right)\right) .
\end{aligned}
$$

\footnotetext{
${ }^{19}$ To be precise, (8.4) obeys the $\propto \gamma_{q \dot{q}_{3}}^{I J K}$ part of also (4.33), while its $\propto \gamma_{q \dot{q} 3}^{I}$ part fixes the form of $\mathcal{A}_{I_{1} I_{2} I_{3}}$.
} 
Here

$$
\begin{aligned}
& \widehat{K}_{21}^{=I}\left(z_{12}\right)=K_{21}^{=I}-\frac{z_{12} q_{(1)}^{I}}{\rho_{1}^{\#}}=K_{21}^{=I}-q_{(1)}^{I} \frac{\left(\vec{K}_{\overline{21}}\right)^{2}}{2 \vec{q}_{(1)} \vec{K}_{21}^{\overline{\bar{l}}}}, \quad \widehat{K}_{31}^{=I}\left(z_{13}\right)=K_{31}^{=I}-q_{(1)}^{I} \frac{\left(\vec{K}_{\overline{31}}\right)^{2}}{2 \vec{q}_{(1)} \vec{K}_{\overline{31}}}, \\
& \widehat{K}_{43}^{=I}\left(z_{12}\right)=K_{43}^{=I}-\frac{z_{12} q_{(4)}^{I}}{\rho_{4}^{\#}}=K_{43}^{=I}-q_{(4)}^{I} \frac{\left(\vec{K}_{21}\right)^{2}}{2 \vec{q}_{(1)} \vec{K}_{21}^{\overline{ }}}, \quad \widehat{K}_{42}^{=I}\left(z_{13}\right)=K_{42}^{=I}-q_{(4)}^{I} \frac{\left(\vec{K}_{31}\right)^{2}}{2 \vec{q}_{(1)} \vec{K}_{\overline{31}}},
\end{aligned}
$$

and $q_{(1)}^{I}, q_{(4)}^{I}$ are projectors of the same complex null ten-vector (6.37) on the first and fourth frames, respectively,

$$
q^{a}=u_{1}^{a I} q_{(1)}^{I}=u_{4}^{a I} q_{(4)}^{I}, \quad q^{a} q_{a}=0, \quad q^{a} u_{a 1}^{=}=0=q^{a} u_{a 4}^{=} .
$$

The $\mathrm{SO}(8)$ complex vectors $q_{(1)}^{I}$ and $q_{(4)}^{I}$ has vanishing squares (7.59) which implies that the vectors (8.7) and (8.8) are also null.

The above calculation demonstrates either incompleteness of our prescription for $\mathrm{BCFW}$ deformation or a need to improve our candidate $\mathrm{BCFW}$ recurrent relations. The problem is that the amplitude calculated with the candidate BCFW relations happens to be apparently dependent on the deformation null-vector $q^{a}$ obeying (6.37). On one hand, using the experience of the $4 \mathrm{D}$ calculation, we might expect a necessity to specify some particular solution of (6.37) in terms of spinor helicity/spinor frame variables corresponding to 1-st and $n$-th (4-th) particle. (We will discuss such a possibility below).

On the other hand, this might indicate that a change of prescription or modification of the proposed candidate BCFW type recurrent relations is necessary. Notice that we did not prove the validity of these relations, but only proposed them as a reasonable candidate. Moreover, the general D-dimensional arguments of $[95,96]$ did not allow to prove the existence of $\mathrm{BCFW}$ relations for $\mathrm{n}$ point $\mathrm{n}$-fermionic amplitudes: they argued their existence for a particular contractions with the deformation vectors (!) of an amplitude with at least one gluon in the case of YM and at least one graviton in the case of gravity (chosen to have a deformed momentum to this end). Thus the first thing to check now is what will be the situation with such an amplitude in 10D SYM case.

\subsection{From the candidate BCFW relation to an expression for 10D SYM with two bosonic and two fermionic legs. Deformation vector dependence}

Let us try to reproduce the 4 point tree amplitude with two bosonic and two fermionic legs from the candidate BCFW relation for 10D SYM. We start from the general relation (which has not been proved to be valid but are obtained from the reasonable candidate (6.57))

$$
\begin{aligned}
\mathcal{A}_{I_{1} \dot{q}_{2} \dot{q}_{3} I_{4}} & \left(k_{1} ; k_{2} ; k_{3} ; k_{4}\right)= \\
= & 2 \mathcal{A}_{z_{12} I_{1} \dot{q}_{2} \dot{q}}\left(\widehat{k_{1}} ; k_{2} ; \widehat{\rho^{\#}}\left(z_{12}\right), v_{q}^{-}\left(z_{12}\right)\right) \\
& \times \frac{1}{\left(P_{12}\right)^{2} \widehat{\rho^{\#}}\left(z_{12}\right)} \mathcal{A}_{z_{12} \dot{q} \dot{q}_{3} I_{4}}\left(-\widehat{\rho^{\#}}\left(z_{12}\right), v_{q}^{-}\left(z_{12}\right) ; k_{3} ; \widehat{k_{4}}\right)-(2 \longleftrightarrow 3) .
\end{aligned}
$$


Let us stress that, although it is not reflected in the notation for l.h.s., the r.h.s. of this relation apparently depends on the deformation vector $q^{a}=-q_{(1)}^{I} u_{1}^{a I}=-q_{(4)}^{I} u_{4}^{a I}$ (7.59). This is the origin of a problem which is possibly common for higher dimensional generalizations of BCFW relations: for $D>4$ the deformation vector is not fixed uniquely. ${ }^{20}$ In $D=4$ case the deformation vector is constructed from the helicity spinors of two particles with deformed momenta in a unique way [16] and the problem does not occur.

Substituting the expressions (8.3) (the cyclic property of the amplitudes implies $\left.\mathcal{A}_{\dot{q}_{1} \dot{q}_{2} I}(1,2,3)=\mathcal{A}_{\dot{I}_{1} \dot{q}_{2}}(3,1,2)\right)$ and using $(7.57)$ and $\widehat{v_{\dot{q}}^{-\alpha}}\left(z_{12}\right) \widehat{v_{\dot{q}}^{-\beta}}\left(z_{12}\right)=\frac{1}{2} \widehat{u_{a}^{\bar{a}}}\left(z_{12}\right) \tilde{\sigma}^{a \alpha \beta}$ (see (3.26)) for the contraction of the variables corresponding to intermediate state, we arrive at

$$
\begin{aligned}
& \mathcal{A}_{I_{1} \dot{q}_{2} \dot{q}_{3} I_{4}}\left(k_{1} ; k_{2} ; k_{3} ; k_{4} \mid q\right)= \\
& \quad=\frac{1}{16 s} \rho_{(2)}^{\#} \rho_{(3)}^{\#} \widehat{u_{a 1}^{I_{1}}} v_{\dot{q}_{2} 2}^{-\alpha}\left(\sigma^{a} \tilde{\sigma}^{b} \sigma^{c}\right)_{\alpha \beta} v_{\dot{q}_{3} 3}^{-\beta} \widehat{u_{b}}\left(z_{12}\right) \widehat{u_{c 4}^{I_{4}}}-(2 \leftrightarrow 3) .
\end{aligned}
$$

Here we have explicitly written the dependence of l.h.s. on the complex deformation nullvector (7.59). This enters $\widehat{u_{b}}\left(z_{12}\right)=\frac{1}{\rho_{1}^{\#}+\rho_{2}^{\#}}\left(\widehat{k_{1 a}}+k_{2 a}\right)$ and also $\widehat{u_{a 1}^{I_{1}}}$ and $\widehat{u_{a 1}^{I_{1}}}(7.67)$ (which are also taken at $z=z_{12}$ ). Furthermore, as one can see from (7.62), a contraction of the deformation vector also enters the denominator of $z_{12}$.

At this point we would like to exploit the result of $[95,96]$ stating that the BCFW type recurrent relations are valid for a particular type of amplitude. Particularly, they are valid for calculation of some amplitudes of the processes with vector particles, one of which, say the first, is chosen to have a deformed momentum. More specifically, BCFW relations are valid for contraction of such amplitude with the deformation vector on its first vector index. In our formalism this corresponds to the contraction of the first $\operatorname{SO}(D-2)$ vector index of the amplitude with $q_{(1)}^{I_{1}}$ component of the deformation vector. Thus a potentially valid contraction of the relation (8.10) reads

$$
\begin{aligned}
& q_{(1)}^{I_{1}} \mathcal{A}_{I_{1} \dot{q}_{2} \dot{q}_{3} I_{4}}\left(k_{1} ; k_{2} ; k_{3} ; k_{4} \mid q\right)= \\
& =\frac{1}{16 s} \rho_{(2)}^{\#} \rho_{(3)}^{\#} q_{a} v_{\dot{q}_{2} 2}^{-\alpha}\left(\sigma^{a} \tilde{\sigma}^{b} \sigma^{c}\right)_{\alpha \beta} v_{\dot{q}_{3} 3}^{-\beta} \widehat{u_{b}}\left(z_{12}\right) \widehat{u_{c 4}}-(2 \leftrightarrow 3) \\
& =-\frac{1}{16 s} \frac{\rho_{(2)}^{\#} \rho_{(3)}^{\#}}{\rho_{1}^{\#}+\rho_{2}^{\#}} q_{a} v_{\dot{q}_{2} 2}^{-\alpha}\left(\sigma^{a} \tilde{\sigma}^{b} \sigma^{c}\right)_{\alpha \beta} v_{\dot{q}_{3} 3}^{-\beta}\left(k_{b 1}+k_{b 2}\right)\left(u_{c 4}^{I_{4}}-\frac{2 z_{12}}{2 \rho_{4}^{\#}} q_{(4)}^{I_{4}} u_{c 4}^{\#}\right)-(2 \leftrightarrow 3) .
\end{aligned}
$$

The passage to the second equality in (8.11) uses the momentum conservation in the first amplitude in the r.h.s. of (8.9), and the fact that the contribution of the deformation vector in $\widehat{k_{b 1}}$ vanishes identically $\left(q_{a}\left(\sigma^{a} \tilde{\sigma}^{b} \sigma^{c}\right) q_{b} \equiv 0\right)$. In $[95,96]$ the interpretation of the relations similar to (8.11) is based on identification of the deformation vector with polarization vector of the first particle.

\footnotetext{
${ }^{20}$ The freedom in choice of a normalized complex null vector defined up to a phase transformation can be associated to the coset $\frac{\mathrm{SO}(D-2)}{\mathrm{SO}(D-4) \otimes \mathrm{U}(1)}$ of dimension $2(D-4)$. See e.g. [53].
} 
Now, the 'covariantization' by the method of [97], namely restoration of the $(\mathrm{SO}(D-2))$ covariant amplitude by just extracting a coefficient contracted with $q_{(1)}^{I_{1}}$, naïvly would give us

$$
\begin{aligned}
& \mathcal{A}_{I_{1} \dot{q}_{2} \dot{q}_{3} I_{4}}\left(k_{1} ; k_{2} ; k_{3} ; k_{4} \mid q\right)= \\
& \quad=\mathcal{A}_{I_{1} \dot{q}_{2} \dot{q}_{3} I_{4}}\left(k_{1} ; k_{2} ; k_{3} ; k_{4}\right)-\frac{2 z_{12}}{2 \rho_{4}^{\#}} q_{(4)}^{J} D_{4}^{\# J} \mathcal{A}_{I_{1} \dot{q}_{2} \dot{q}_{3} I_{4}}\left(k_{1} ; k_{2} ; k_{3} ; k_{4}\right),
\end{aligned}
$$

where $D_{i}^{\# J}=u_{a i}^{\#} \frac{\partial}{\partial u_{a i}^{I}}-u_{a i}^{I} \frac{\partial}{\partial u_{a i}^{\bar{E}}}$ is one of the harmonic covariant derivatives and

$$
\begin{aligned}
& \mathcal{A}_{I_{1} \dot{q}_{2} \dot{q}_{3} I_{4}}\left(k_{1} ; k_{2} ; k_{3} ; k_{4}\right)=\mathcal{A}_{I_{1} \dot{q}_{2} \dot{q}_{3} I_{4}}\left(k_{1} ; k_{2} ; k_{3} ; k_{4} \mid 0\right)= \\
& =-\frac{1}{16 s} \frac{\rho_{(2)}^{\#} \rho_{(3)}^{\#}}{\rho_{1}^{\#}+\rho_{2}^{\#}} u_{a 1}^{I_{1}} v_{\dot{q}_{2} 2}^{-\alpha}\left(\sigma^{a} \tilde{\sigma}^{b} \sigma^{c}\right)_{\alpha \beta} v_{\dot{q}_{3} 3}^{-\beta}\left(k_{b 1}+k_{b 2}\right) u_{c 4}^{I_{4}}-(2 \leftrightarrow 3) .
\end{aligned}
$$

Just this part of our BCFW amplitude has the structure close to the one of the matrix elements described in [103].

However, the presence of the second term in (8.12) brings us back to the problem of an unwanted dependence on a deformation vector $q^{a}$, which, in contradistinction to $4 \mathrm{D}$ case, is not fixed uniquely neither in our formalism, nor in generic discussion of higher dimensional, $D>4$ BCFW relations presented in [95, 96].

Coming back to (8.11) where the deformation vector is considered as polarization vector of the first particle does not resolve the issue. As for $D>4$ the properties of the deformation vector do not fix it uniquely, the apparent problem is how to treat the freedom in its choice. If we assume that (8.11) is valid for an arbitrary consistent choice of the deformation vector, the (appropriately defined ${ }^{21}$ ) derivative of (8.11) with respect to $q_{(1)}^{I}$ gives us an additional relation for the amplitudes. Probably in such a way one can reach an algorithmic formulation of the above mentioned covariantization procedure suggested in [97], but consistency of this is to be investigated.

Perhaps this problem can be solved by exploiting the relation of the present approach with the analytic superamplitude formalism proposed recently in [53]. Indeed, as it was shown in conclusion of [53], in its frame the freedom in choosing the deformation vector can be reduced to one complex number, like it is the case for the deformations of Weyl spinors used in the study of $4 \mathrm{D}$ superamplitudes. To be more precise, the deformation vector is expressed there in terms of complex spinor helicity variables constructed form the spinor frame variables and internal harmonic variables parametrizing $\mathrm{SO}(D-2) /[\mathrm{SO}(D-4) \otimes \mathrm{U}(1)]$ coset, and thus it can be identified with a coordinate of such a coset. This brings us back to the problem of whether one should allow to differentiate the deformation vector in (8.11) to obtain a new relation for the amplitude, which have to be investigated.

This and, more generally, the use of interplay between the present constrained and the analytic superamplitude formalisms for their mutual development will be the subject of future work.

\footnotetext{
${ }^{21}$ In the light of the statement in footnote 20 , this should be an appropriate covariant derivative on the coset $\frac{\mathrm{SO}(D-2)}{\mathrm{SO}(D-4) \otimes \mathrm{U}(1)}$.
} 


\section{Conclusion and outlook}

In this paper we have developed spinor helicity formalism for 11D supergravity (SUGRA) and on-shell superfield formalism for tree amplitudes of 11D SUGRA and 10D SYM. Another superamplitude formalism for 10D SYM was proposed some years ago in [25]. It was based on Clifford superfields and looks quite nonminimal and difficult to use. In contrast, the 10D spinor helicity formalism of [25] coincide with ours; in this paper we clarify its structure and the nature of constrained spinor helicity variables.

The observation that the basic variables of the 10D spinor helicity formalism of [25] coincide with spinor moving frame variables (Lorentz harmonics), allowed us to develop a more economic superfield description of 10D SYM amplitudes and also to obtain the 11D generalization of the spinor helicity and superamplitude formalisms. Our superamplitudes are multiparticle generalizations of constrained superfields describing the linearized 11D SUGRA and 10D SYM which were found in [35]. They obey a number of equations, hence the name of constrained superamplitude formalism which we use for our approach.

We have shown how the constrained on-shell superfields of [35] can be obtained from quantization of 11D and 10D massless superparticle mechanics. Actually we have used the massless superparticle mechanics and its quantization as a guide for the development of the superamplitude formalism.

The constrained $n$-particle superamplitudes of 10D SYM (11D SUGRA) depend on the set of spinor helicity variables as well as on the set of fermionic variables $\theta_{q i}^{-}, q=1, \ldots, 8$ $(q=1, \ldots, 16), i=1, \ldots, n$. The spinor helicity variables include the set of constrained spinors $v_{\alpha q i}^{-}, \alpha=1, \ldots, 16(\alpha=1, \ldots, 32)$, spinor frame variables or Lorentz harmonics, parametrizing celestial sphere $\mathbb{S}^{8}\left(\mathbb{S}^{9}\right)$ and densities $\rho_{i}^{\#}, i=1, \ldots, n$, which are allowed to be negative. We have described the set of equations, which are imposed on constrained superamplitudes and restrict their dependence on $\theta_{q i}^{-}$.

We have made some stages towards calculation of superamplitudes and amplitudes in this formalism. In particular, we have found a gauge fixed expressions for the Lorentz harmonics which can be considered as a covariant counterpart of the light cone gauge and promises to be a very useful tool for the calculation of amplitudes and superamplitudes. We have also considered the consequences of the momentum conservation and described supermomentum, the fermionic superpartner of total momentum, in the spinor frame formalism.

We have obtained the supersymmetric Ward identities for 10D SYM and 11D SUGRA amplitudes and used that to check our guess for 3-point 10D amplitude with two fermions. Such a calculation is simplified in the above described gauge fixed on the spinor frame variables.

We have also discussed a natural candidate for the BCFW-type recurrent relations for our constrained superamplitudes. Setting all the fermionic variables equal to zero, we reduce these candidate BCFW-type relations for superamplitudes to the relations for amplitudes. As a check of completeness and consistency, we used the above 3-point amplitudes and the candidate BCFW relations to obtain a gauge fixed form of the 10D 4-fermion tree amplitude and also a covariant form of the 4 point amplitude with 2 fermionic and 2 bosonic legs. 
This simple calculation however showed a problem indicating either incompleteness of the higher dimensional BCFW deformation prescription or a need for improvement of our candidate BCFW recurrent relations. The above 4-point tree amplitudes calculated with their use suffer an apparent dependence on a deformation null-vector, which, in contradistinction to $4 \mathrm{D}$ case, is defined with a big degree of arbitrariness in $\mathrm{D}=10$ and $\mathrm{D}=11$.

One can observe that the deformation null-vector $q^{a}=q_{(1)}^{I} u_{1}^{a I}$ can be associated with breaking of the $\mathrm{SO}(D-2)_{1}$ gauge symmetry of the real spinor frame formalism down to its $\mathrm{SO}(D-4) \times \mathrm{U}(1)$ subgroup. Similar symmetry breaking occurs inevitably in the analytic superfield approach of [53]. It is related to the appearance of complex structure characteristic for the analytic superamplitude formalism and complex spinor frame variables used in it. As it was shown in [53], the BCFW deformation of complex spinor frame and fermionic variables of the analytic superfield formalism can be quite naturally fixed uniquely up to a single complex number $z$, i.e. can reproduce the structure similar to the known from $4 \mathrm{D}$ case. This suggests that, perhaps, a solution of the above discussed problem with candidate $\mathrm{BCFW}$ relations of the constrained superamplitude formalism can be found by elaborating its interrelation with the analytic superamplitude approach.

This issue is clearly the first in the que of problems to be addressed for further development of our approach to tree superamplitudes in $\mathrm{D}=10$ and $\mathrm{D}=11$, which will be the subject of forthcoming work. Upon solving it, it would be also natural to search for a generalization of our formalism for the case of loop superamplitudes. ${ }^{22}$

Another interesting direction of study is to approach the constrained superamplitude formalism by quantizing 11D and 10D Green-Schwarz type counterparts of the so-called ambitwistor string. ${ }^{23}$ This might lead us to a convenient superfield form of the scattering equation approach [109-111].

\section{Acknowledgments}

This work has been supported in part by the Spanish Ministry of Economy, Industry and Competitiveness (MINECO) grant FPA 2015-66793-P, which is partially financed with FEDER/ERDF (European Regional Development Fund of the European Union), by the Basque Government Grant IT-979-16, and the Basque Country University program UFI $11 / 55$.

The author is thankful to the Theoretical Department of CERN (Geneva, Switzerland), to the Galileo Galilei Institute for Theoretical Physics and the INFN (GGI, Florence, Italy), to the organizers of the GGI program "Supergravity: what next?", and especially to Toine

\footnotetext{
${ }^{22}$ Despite of neither 10D SYM nor 11D supergravity is expected to be finite, a number of interesting problems can be addressed by studying their amplitudes: see for instance [98, 99] as well as recent [100] and refs. therein.

${ }^{23}$ Ambitwistor string $[107,108]$ is known to reproduce the scattering equation of Cachazo, He and Yuan (CHY) [109-111]. Originally the ambitwistor string was formulated as a Nevieu-Scwarz-Ramond (NSR) type model in $\mathrm{d}=10$. However, as it was discussed in [112], at the classical level this model is equivalent to the so-called null-superstring and thus (see [113]) to the twistor string [1, 114, 115]; hence it should allow for a consistent quantization in an arbitrary number of dimensions. The $\mathrm{D}=4$ ambitwistor string models have been developed in [116-119]. See also [21, 120-122] for related results.
} 
Van Proyeen, as well as to the Simons Center for Geometry and Physics, Stony Brook University (New York, US) for the hospitality and partial support of his visits at certain stages of this work.

The author is grateful to Dima Sorokin and Emeri Sokatchev for useful discussions and suggestions, and to Luis Alvarez-Gaume, Paolo Di Vecchia and Boris Piolin for useful discussions on related topics.

\section{A Clifford superfield version of $\mathrm{D}=10$ superamplitudes and fermionic BCFW deformation}

\section{A.1 SUSY generator representation with Clifford algebra element}

In this appendix we will show how the Clifford superfield form of the on-shell superfield formalism, a one-particle counterpart to the approach to 10D superamplitudes proposed by Carron-Huot and O'Connell in [25], and its 11D generalization, can be obtained from covariant quantization of the massless superparticle model in its spinor moving frame formulation.

As we have discussed in section 5.5, In the 'analytic basis' the 11D massless superparticle has only second class fermionic constraints (5.31) which obey

$$
\left\{d_{q}^{+}, d_{p}^{+}\right\}_{P . B .}=-4 i \rho^{\#} \delta_{q p} .
$$

If we pass to the Dirac brackets, the fermionic coordinate variable will obey

$$
\left\{\theta_{q}^{-}, \theta_{p}^{-}\right\}_{D . B .}=-\frac{i}{4 \rho^{\#}} \delta_{q p}
$$

so that after quantization the algebra of fermionic operators $\hat{\theta}_{q}^{-}$is

$$
\left\{\hat{\theta}_{q}^{-}, \hat{\theta}_{p}^{-}\right\}=\frac{1}{4 \rho^{\#}} \delta_{q p} .
$$

Thus they can be identified with the 16-dimensional Clifford algebra generators

$$
\hat{\theta}_{q}^{-}=\frac{1}{\sqrt{2 \rho^{\#}}} \mathfrak{C}_{q}, \quad\left\{\mathfrak{C}_{q}, \mathfrak{C}_{p}\right\}=2 \delta_{q p}
$$

The superfield formalism by Carron-Huot and O'Connell is constructed by considering wavefunctions dependent on Clifford variable $\mathfrak{C}_{q}$ (we call this "Clifford superfield approach").

The realization of the $\mathrm{D}=10$ supersymmetry generator on the state with light-like momentum $k_{a}$, used in [25] can be written as (see (A.8))

$$
Q_{\alpha}=\sqrt{\rho^{\#}} v_{\alpha q}^{-} \mathfrak{C}_{q}
$$

Here $v_{\alpha q}{ }^{-}$are the square roots of $k_{a}$ as defined by (4.6), which implies $k_{a}=\frac{1}{8} \rho^{\#} v_{q}^{-} \tilde{\sigma} v_{q}^{-}$. It is easy to check that

$$
\left\{Q_{\alpha}, Q_{\beta}\right\}=2 \rho^{\#} v_{\alpha q}{ }^{-} v_{\beta q}{ }^{-}=\sigma_{\alpha \beta}^{a} k_{a} .
$$


The same equations with $\alpha, \beta=1, \ldots, 32, \quad q, p=1, \ldots, 16$ and $\sigma^{a} \mapsto \Gamma^{a}$ with $a=$ $0,1, \ldots, 10$ describe the realization of $11 \mathrm{D}$ supersymmetry generator in terms of 16 dimensional Clifford algebra and the homogeneous coordinate of $\mathbb{S}^{9}$.

In $\mathrm{D}=10$ case, one can realize these generators on fourier images of the fields $w^{I}=w^{I}\left(x^{=}, v_{\alpha q}^{-}\right)$and $\psi_{q}=\psi_{q}\left(x^{=}, v_{\alpha q}^{-}\right)$(see (4.16), (4.15), (4.17))

$$
\mathfrak{C}_{q} \psi_{\dot{q}}\left(\rho^{\#}\right)=\frac{1}{\sqrt{\rho^{\#}}} \gamma_{q \dot{q}}^{I} w^{I}\left(\rho^{\#}\right), \quad \mathfrak{C}_{q} w^{I}\left(\rho^{\#}\right)=\gamma_{q \dot{q}}^{I} \sqrt{\rho^{\#}} \psi_{\dot{q}}\left(\rho^{\#}\right)
$$

We can also write the formal supersymmetry generator acting on the states of $n$ on-shell particles and on n-point amplitudes [25]:

$$
Q_{\alpha}=\sum_{i=1}^{n} \sqrt{\rho_{(i)}^{\#}} v_{\alpha q(i)}^{-} \mathfrak{C}_{q(i)} .
$$

Here $\mathfrak{C}_{q(i)}$ are $n$ sets of generators of $8 \mathrm{~d}$ Clifford algebra (16d Clifford algebra in the case of $\mathrm{D}=11$ ),

$$
\left\{\mathfrak{C}_{q(i)}, \mathfrak{C}_{p(j)}\right\}=2 \delta_{(i)(j)} \delta_{q p} \mathcal{I} .
$$

It is easy to check that these supersymmetry generator obey the usual supersymmetry algebra (A.6),

$$
\left\{Q_{\alpha}, Q_{\beta}\right\}=2 P_{\alpha \beta}, \quad P_{\alpha \beta}=\sigma_{\alpha \beta}^{a} P_{a}=\sum_{i=1}^{n} \rho_{(i)}^{\#} v_{\alpha q(i)}^{-} v_{\beta q(i)}^{-}
$$

the right hand side of which contains the total momentum, the sum of momenta of all the scattered particle. Notice that this vanishes due to the momentum conservation in scattering processes, so that on the mass shell the $10 \mathrm{D}$ supersymmetry generator (A.8) is just nilpotent,

$$
P_{a}=\sum_{i=1}^{n} k_{a(i)}=0 \quad \Rightarrow \quad\left\{Q_{\alpha}, Q_{\beta}\right\}=0 .
$$

\section{A.2 Clifford superfield version of $\mathrm{D}=10 \mathrm{BCFW}$ deformation}

The first generalization of the BCFW-type deformation of 10D on-shell superamplitudes was proposed in [25], where the amplitudes depending on the spinor helicity variables (which are, as we have seen, essentially the spinor moving frame variables, $v_{\alpha q(i)}$ ) and Clifford algebra variables $\mathfrak{C}_{q}$ were considered. We call these $\mathcal{A}\left(\rho_{(1)}^{\#}, v_{\alpha q(1)}, \mathfrak{C}_{(1)} ; \ldots, \rho_{(n)}^{\#}, v_{\alpha q(n)}\right.$, $\left.\mathfrak{C}_{(n)}\right)$ Clifford superamplitudes.

The BCFW-type deformation of 10D spinor helicity variables from [25] can be reproduced from the deformation of spinor frame variables described in (6.34) and (6.35). Here for completeness we present (in our notation) also the deformation of the Clifford algebra variables proposed in [25]. It reads

$$
\begin{aligned}
& \widehat{\mathfrak{C}_{p(n)}}=\mathfrak{C}_{p(n)}+z \mathfrak{C}_{q(1)} \mathbb{M}_{q p}=e^{\frac{z}{2} \mathfrak{c}_{q(1)}} \mathbb{M}_{q p} \mathfrak{C}_{p(n)} \mathfrak{C}_{p(n)} e^{-\frac{z}{2} \mathfrak{C}_{q(1)}} \mathbb{M}_{q p} \mathfrak{c}_{p(n)} \\
& \widehat{\mathfrak{C}_{q(1)}}=\mathfrak{C}_{q(1)}-z \mathbb{M}_{q p} \mathfrak{C}_{p(n)}=e^{\frac{z}{2} \mathfrak{C}_{q(1)}} \mathbb{M}_{q p} \mathfrak{C}_{p(n)} \mathfrak{C}_{q(1)} e^{-\frac{z}{2} \mathfrak{C}_{q(1)}} \mathbb{M}_{q p} \mathfrak{C}_{p(n)}
\end{aligned}
$$

where the matrix $\mathbb{M}_{q p}$ is nilpotent, (6.40). 
Open Access. This article is distributed under the terms of the Creative Commons Attribution License (CC-BY 4.0), which permits any use, distribution and reproduction in any medium, provided the original author(s) and source are credited.

\section{References}

[1] E. Witten, Perturbative gauge theory as a string theory in twistor space, Commun. Math. Phys. 252 (2004) 189 [hep-th/0312171] [INSPIRE].

[2] R. Penrose, Twistor algebra, J. Math. Phys. 8 (1967) 345 [InSPIRE].

[3] R. Penrose and M.A.H. MacCallum, Twistor theory: An Approach to the quantization of fields and space-time, Phys. Rept. 6 (1972) 241 [InSPIRE].

[4] R. Penrose and W. Rindler, Spinors And Space-time. Volume 1. Two-Spinor Calculus and Relativistic Fields, Cambridge University Press (1984).

[5] R. Penrose and W. Rindler, Spinors And Space-time. Volume 2. Spinor And Twistor Methods In Space-time Geometry, Cambridge University Press (1986).

[6] A. Ferber, Supertwistors and Conformal Supersymmetry, Nucl. Phys. B 132 (1978) 55 [INSPIRE].

[7] T. Shirafuji, Lagrangian Mechanics of Massless Particles With Spin, Prog. Theor. Phys. 70 (1983) 18 [INSPIRE].

[8] E. Witten, An Interpretation of Classical Yang-Mills Theory, Phys. Lett. B 77 (1978) 394 [INSPIRE].

[9] Z. Bern, J.J. Carrasco, L.J. Dixon, H. Johansson and R. Roiban, Amplitudes and Ultraviolet Behavior of $N=8$ Supergravity, Fortsch. Phys. 59 (2011) 561 [arXiv:1103.1848] [INSPIRE].

[10] J.M. Drummond, J. Henn, G.P. Korchemsky and E. Sokatchev, Dual superconformal symmetry of scattering amplitudes in $N=4$ super-Yang-Mills theory, Nucl. Phys. B 828 (2010) 317 [arXiv:0807.1095] [INSPIRE].

[11] J.M. Drummond, J.M. Henn and J. Plefka, Yangian symmetry of scattering amplitudes in $N=4$ super Yang-Mills theory, JHEP 05 (2009) 046 [arXiv:0902.2987] [INSPIRE].

[12] B. Eden, P. Heslop, G.P. Korchemsky and E. Sokatchev, The super-correlator/super-amplitude duality: Part II, Nucl. Phys. B 869 (2013) 378 [arXiv:1103.4353] [INSPIRE].

[13] R. Kallosh and T. Ortín, New Ery invariants and amplitudes, JHEP 09 (2012) 137 [arXiv: 1205.4437] [INSPIRE].

[14] H. Elvang and Y.t. Huang, Scattering Amplitudes in Gauge Theory and Gravity, Cambridge University Press (2015.

[15] N. Arkani-Hamed, J.L. Bourjaily, F. Cachazo, A.B. Goncharov, A. Postnikov and J. Trnka, Grassmannian Geometry of Scattering Amplitudes, Cambridge University Press (2015).

[16] R. Britto, F. Cachazo, B. Feng and E. Witten, Direct proof of tree-level recursion relation in Yang-Mills theory, Phys. Rev. Lett. 94 (2005) 181602 [hep-th/0501052] [INSPIRE].

[17] R. Britto, F. Cachazo and B. Feng, New recursion relations for tree amplitudes of gluons, Nucl. Phys. B 715 (2005) 499 [hep-th/0412308] [INSPIRE]. 
[18] M. Bianchi, H. Elvang and D.Z. Freedman, Generating Tree Amplitudes in $N=4$ SYM and $N=8 S G$, JHEP 09 (2008) 063 [arXiv:0805.0757] [InSPIRE].

[19] N. Arkani-Hamed, F. Cachazo and J. Kaplan, What is the Simplest Quantum Field Theory?, JHEP 09 (2010) 016 [arXiv:0808.1446] [INSPIRE].

[20] A. Brandhuber, P. Heslop and G. Travaglini, A Note on dual superconformal symmetry of the $N=4$ super Yang-Mills S-matrix, Phys. Rev. D 78 (2008) 125005 [arXiv:0807.4097] [INSPIRE].

[21] P. Heslop and A.E. Lipstein, On-shell diagrams for $\mathcal{N}=8$ supergravity amplitudes, JHEP 06 (2016) 069 [arXiv: 1604.03046] [INSPIRE].

[22] E. Herrmann and J. Trnka, Gravity On-shell Diagrams, JHEP 11 (2016) 136 [arXiv: 1604.03479] [INSPIRE].

[23] A. Hodges, Eliminating spurious poles from gauge-theoretic amplitudes, JHEP 05 (2013) 135 [arXiv:0905.1473] [INSPIRE].

[24] L.J. Mason and D. Skinner, Dual Superconformal Invariance, Momentum Twistors and Grassmannians, JHEP 11 (2009) 045 [arXiv:0909.0250] [INSPIRE].

[25] S. Caron-Huot and D. O'Connell, Spinor Helicity and Dual Conformal Symmetry in Ten Dimensions, JHEP 08 (2011) 014 [arXiv: 1010.5487] [INSPIRE].

[26] R.H. Boels and D. O'Connell, Simple superamplitudes in higher dimensions, JHEP 06 (2012) 163 [arXiv: 1201.2653] [inSPIRE].

[27] R.H. Boels, Maximal R-symmetry violating amplitudes in type IIB superstring theory, Phys. Rev. Lett. 109 (2012) 081602 [arXiv:1204.4208] [INSPIRE].

[28] Y. Wang and X. Yin, Constraining Higher Derivative Supergravity with Scattering Amplitudes, Phys. Rev. D 92 (2015) 041701 [arXiv:1502.03810] [INSPIRE].

[29] Y. Wang and X. Yin, Supervertices and Non-renormalization Conditions in Maximal Supergravity Theories, arXiv:1505.05861 [INSPIRE].

[30] I. Bandos, Britto-Cachazo-Feng-Witten-Type recurrent relations for tree amplitudes of $D=11$ supergravity, Phys. Rev. Lett. 118 (2017) 031601 [arXiv:1605.00036] [INSPIRE].

[31] A.S. Galperin, P.S. Howe and K.S. Stelle, The Superparticle and the Lorentz group, Nucl. Phys. B 368 (1992) 248 [hep-th/9201020] [INSPIRE].

[32] F. Delduc, A. Galperin and E. Sokatchev, Lorentz harmonic (super)fields and (super)particles, Nucl. Phys. B 368 (1992) 143 [InSPIRE].

[33] I.A. Bandos and A.Yu. Nurmagambetov, Generalized action principle and extrinsic geometry for $N=1$ superparticle, Class. Quant. Grav. 14 (1997) 1597 [hep-th/9610098] [INSPIRE].

[34] D.V. Uvarov, Spinor description of D $=5$ massless low-spin gauge fields, Class. Quant. Grav. 33 (2016) 135010 [arXiv: 1506.01881] [INSPIRE].

[35] A.S. Galperin, P.S. Howe and P.K. Townsend, Twistor transform for superfields, Nucl. Phys. B 402 (1993) 531 [INSPIRE].

[36] I.A. Bandos, J.A. de Azcarraga and D.P. Sorokin, On D = 11 supertwistors, superparticle quantization and a hidden $\mathrm{SO}(16)$ symmetry of supergravity, in Proceedings, 22nd Max Born Symposium on Quantum, Super and Twistors: A Conference in Honor of Jerzy Lukierski on His 70th Birthday, Wroclaw, Poland, September 27-29, 2006 (2006) [hep-th/0612252] [INSPIRE]. 
[37] I.A. Bandos, Spinor moving frame, M0-brane covariant BRST quantization and intrinsic complexity of the pure spinor approach, Phys. Lett. B 659 (2008) 388 [arXiv:0707.2336] [INSPIRE].

[38] I.A. Bandos, $D=11$ massless superparticle covariant quantization, pure spinor BRST charge and hidden symmetries, Nucl. Phys. B 796 (2008) 360 [arXiv:0710.4342] [INSPIRE].

[39] I.A. Bandos and A.A. Zheltukhin, Spinor Cartan moving n hedron, Lorentz harmonic formulations of superstrings and kappa symmetry, JETP Lett. 54 (1991) 421 [INSPIRE].

[40] I.A. Bandos and A.A. Zheltukhin, Green-Schwarz superstrings in spinor moving frame formalism, Phys. Lett. B 288 (1992) 77 [INSPIRE].

[41] I.A. Bandos and A.A. Zheltukhin, Twistor-like approach in the Green-Schwarz D = 10 superstring theory, Phys. Part. Nucl. 25 (1994) 453 [INSPIRE].

[42] I.A. Bandos and A.A. Zheltukhin, Generalization of Newman-Penrose dyads in connection with the action integral for supermembranes in an eleven-dimensional space, JETP Lett. $\mathbf{5 5}$ (1992) 81 [INSPIRE].

[43] I.A. Bandos and A.A. Zheltukhin, Eleven-dimensional supermembrane in a spinor moving repere formalism, Int. J. Mod. Phys. A 8 (1993) 1081 [InSPIRE].

[44] I.A. Bandos and A.A. Zheltukhin, $N=1$ superp-branes in twistor - like Lorentz harmonic formulation, Class. Quant. Grav. 12 (1995) 609 [hep-th/9405113] [INSPIRE].

[45] D.P. Sorokin, V.I. Tkach and D.V. Volkov, Superparticles, Twistors and Siegel Symmetry, Mod. Phys. Lett. A 4 (1989) 901 [inSPIRE].

[46] F. Delduc, A. Galperin, P.S. Howe and E. Sokatchev, A Twistor formulation of the heterotic $D=10$ superstring with manifest $(8,0)$ world sheet supersymmetry, Phys. Rev. D 47 (1993) 578 [hep-th/9207050] [INSPIRE].

[47] F. Delduc, E. Ivanov and E. Sokatchev, Twistor like superstrings with $D=3, D=4, D=6$ target superspace and $N=(1,0), N=(2,0), N=(4,0)$ world sheet supersymmetry, Nucl. Phys. B 384 (1992) 334 [hep-th/9204071] [INSPIRE].

[48] D.P. Sorokin, Superbranes and superembeddings, Phys. Rept. 329 (2000) 1 [hep-th/9906142] [INSPIRE].

[49] I.A. Bandos, D.P. Sorokin, M. Tonin, P. Pasti and D.V. Volkov, Superstrings and supermembranes in the doubly supersymmetric geometrical approach, Nucl. Phys. B 446 (1995) 79 [hep-th/9501113] [INSPIRE].

[50] P.S. Howe and E. Sezgin, $D=11, p=5$, Phys. Lett. B 394 (1997) 62 [hep-th/9611008] [INSPIRE].

[51] I.A. Bandos, K. Lechner, A. Nurmagambetov, P. Pasti, D.P. Sorokin and M. Tonin, Covariant action for the superfive-brane of M-theory, Phys. Rev. Lett. 78 (1997) 4332 [hep-th/9701149] [INSPIRE].

[52] M. Aganagic, J. Park, C. Popescu and J.H. Schwarz, World volume action of the M-theory five-brane, Nucl. Phys. B 496 (1997) 191 [hep-th/9701166] [INSPIRE].

[53] I. Bandos, An analytic superfield formalism for tree superamplitudes in $D=10$ and $D=11$, JHEP 05 (2018) 103 [arXiv: 1705.09550] [INSPIRE].

[54] S. Mandelstam, Light Cone Superspace and the Ultraviolet Finiteness of the $N=4$ Model, Nucl. Phys. B 213 (1983) 149 [INSPIRE]. 
[55] L. Brink, O. Lindgren and B.E.W. Nilsson, $N=4$ Yang-Mills Theory on the Light Cone, Nucl. Phys. B 212 (1983) 401 [InSPIRE].

[56] L. Brink, O. Lindgren and B.E.W. Nilsson, The Ultraviolet Finiteness of the $N=4$ Yang-Mills Theory, Phys. Lett. B 123 (1983) 323 [INSPIRE].

[57] M.B. Green, J.H. Schwarz and L. Brink, Superfield Theory of Type II Superstrings, Nucl. Phys. B 219 (1983) 437 [InSPIRE].

[58] P.S. Howe, K.S. Stelle and P.K. Townsend, Miraculous Ultraviolet Cancellations in Supersymmetry Made Manifest, Nucl. Phys. B 236 (1984) 125 [InSPIRE].

[59] G. Bossard, P.S. Howe and K.S. Stelle, The Ultra-violet question in maximally supersymmetric field theories, Gen. Rel. Grav. 41 (2009) 919 [arXiv:0901.4661] [INSPIRE].

[60] P.S. Howe and U. Lindström, Higher Order Invariants in Extended Supergravity, Nucl. Phys. B 181 (1981) 487 [INSPIRE].

[61] R.E. Kallosh, Counterterms in extended supergravities, Phys. Lett. B 99 (1981) 122 [INSPIRE].

[62] N. Berkovits, Super Poincaré covariant quantization of the superstring, JHEP 04 (2000) 018 [hep-th/0001035] [INSPIRE].

[63] N. Berkovits, Multiloop amplitudes and vanishing theorems using the pure spinor formalism for the superstring, JHEP 09 (2004) 047 [hep-th/0406055] [INSPIRE].

[64] N. Berkovits and N. Nekrasov, Multiloop superstring amplitudes from non-minimal pure spinor formalism, JHEP 12 (2006) 029 [hep-th/0609012] [INSPIRE].

[65] N. Berkovits and H. Gomez, An Introduction to Pure Spinor Superstring Theory, in 9th Summer School on Geometric, Algebraic and Topological Methods for Quantum Field Theory, Villa de Leyva, Colombia, July 20-31, 2015, pp. 221-246 (2017) [DOI:10.1007/978-3-319-65427-0_6] [arXiv: 1711.09966] [INSPIRE].

[66] J. Bjornsson, Multi-loop amplitudes in maximally supersymmetric pure spinor field theory, JHEP 01 (2011) 002 [arXiv: 1009.5906] [InSPIRE].

[67] J. Bjornsson and M.B. Green, 5 loops in 24/5 dimensions, JHEP 08 (2010) 132 [arXiv: 1004.2692] [INSPIRE].

[68] C.R. Mafra and O. Schlotterer, Towards one-loop SYM amplitudes from the pure spinor BRST cohomology, Fortsch. Phys. 63 (2015) 105 [arXiv:1410.0668] [INSPIRE].

[69] C.R. Mafra and O. Schlotterer, Two-loop five-point amplitudes of super Yang-Mills and supergravity in pure spinor superspace, JHEP 10 (2015) 124 [arXiv:1505.02746] [INSPIRE].

[70] M. Cederwall, D = 11 supergravity with manifest supersymmetry, Mod. Phys. Lett. A 25 (2010) 3201 [arXiv: 1001.0112] [INSPIRE].

[71] M. Cederwall and A. Karlsson, Loop amplitudes in maximal supergravity with manifest supersymmetry, JHEP 03 (2013) 114 [arXiv: 1212.5175] [INSPIRE].

[72] N. Berkovits and M. Guillen, Equations of motion from Cederwall's pure spinor superspace actions, JHEP 08 (2018) 033 [arXiv: 1804.06979] [INSPIRE].

[73] A. Karlsson, Ultraviolet divergences in maximal supergravity from a pure spinor point of view, JHEP 04 (2015) 165 [arXiv: 1412.5983] [INSPIRE].

[74] N. Berkovits and S.A. Cherkis, Higher-dimensional twistor transforms using pure spinors, JHEP 12 (2004) 049 [hep-th/0409243] [INSPIRE]. 
[75] N. Berkovits, Ten-Dimensional Super-Twistors and Super-Yang-Mills, JHEP 04 (2010) 067 [arXiv:0910.1684] [INSPIRE].

[76] N. Berkovits, Twistor Origin of the Superstring, JHEP 03 (2015) 122 [arXiv:1409.2510] [INSPIRE].

[77] S.J. Parke and T.R. Taylor, An Amplitude for $n$ Gluon Scattering, Phys. Rev. Lett. 56 (1986) 2459 [INSPIRE].

[78] E. Sokatchev, Light Cone Harmonic Superspace and Its Applications, Phys. Lett. B 169 (1986) 209 [INSPIRE].

[79] E. Sokatchev, Harmonic superparticle, Class. Quant. Grav. 4 (1987) 237 [InSPIRE].

[80] W. Siegel, Hidden Local Supersymmetry in the Supersymmetric Particle Action, Phys. Lett. B 128 (1983) 397 [INSPIRE].

[81] J.A. de Azcarraga and J. Lukierski, Supersymmetric Particles with Internal Symmetries and Central Charges, Phys. Lett. B 113 (1982) 170 [inSPIRE].

[82] A. Galperin, E. Ivanov, S. Kalitsyn, V. Ogievetsky and E. Sokatchev, Unconstrained $N=2$ Matter, Yang-Mills and Supergravity Theories in Harmonic Superspace, Class. Quant. Grav. 1 (1984) 469 [Erratum ibid. 2 (1985) 127] [INSPIRE].

[83] A. Galperin, E. Ivanov, S. Kalitsyn, V. Ogievetsky and E. Sokatchev, Unconstrained Off-Shell $N=3$ Supersymmetric Yang-Mills Theory, Class. Quant. Grav. 2 (1985) 155 [INSPIRE].

[84] A.S. Galperin, E.A. Ivanov, V.I. Ogievetsky and E.S. Sokatchev, Harmonic superspace, Cambridge University Press (2001).

[85] I.A. Bandos, Superparticle in Lorentz harmonic superspace (in Russian), Sov. J. Nucl. Phys. 51 (1990) 906 [INSPIRE].

[86] R.J. Eden, P.V. Landshoff, D.I. Olive and J.C. Polkinghorne, The analytic S-matrix, Cambridge University Press (1966).

[87] P.A.M. Dirac, Lectures on Quantum mechanics, Yeshiva University, New York (1964).

[88] F.A. Berezin and M.S. Marinov, Particle Spin Dynamics as the Grassmann Variant of Classical Mechanics, Annals Phys. 104 (1977) 336 [INSPIRE].

[89] L. Brink, P. Di Vecchia and P.S. Howe, A Lagrangian Formulation of the Classical and Quantum Dynamics of Spinning Particles, Nucl. Phys. B 118 (1977) 76 [INSPIRE].

[90] M.B. Green, M. Gutperle and H.H. Kwon, Light cone quantum mechanics of the eleven-dimensional superparticle, JHEP 08 (1999) 012 [hep-th/9907155] [INSPIRE].

[91] S.S. Schweber, An Introduction to Relativistic Quantum Field Theory, Row, Peterson, Evanston, Ill (1961).

[92] M. Tonin, World sheet supersymmetric formulations of Green-Schwarz superstrings, Phys. Lett. B 266 (1991) 312 [INSPIRE].

[93] M. Tonin, kappa symmetry as world sheet supersymmetry in $D=10$ heterotic superstring, Int. J. Mod. Phys. A 7 (1992) 6013 [inSPIRE].

[94] B.M. Zupnik and D.G. Pak, Differential and Integral Forms in Supergauge Theories and Supergravity, Class. Quant. Grav. 6 (1989) 723 [InSPIRE].

[95] N. Arkani-Hamed and J. Kaplan, On Tree Amplitudes in Gauge Theory and Gravity, JHEP 04 (2008) 076 [arXiv:0801.2385] [INSPIRE]. 
[96] C. Cheung, On-Shell Recursion Relations for Generic Theories, JHEP 03 (2010) 098 [arXiv:0808.0504] [INSPIRE].

[97] C. Cheung and D. O'Connell, Amplitudes and Spinor-Helicity in Six Dimensions, JHEP 07 (2009) 075 [arXiv: 0902.0981] [inSPIRE].

[98] M.B. Green, M. Gutperle and P. Vanhove, One loop in eleven-dimensions, Phys. Lett. B 409 (1997) 177 [hep-th/9706175] [inSPIRE].

[99] S. Deser and D. Seminara, Tree amplitudes and two loop counterterms in D $=11$ supergravity, Phys. Rev. D 62 (2000) 084010 [hep-th/0002241] [INSPIRE].

[100] M.B. Green and A. Rudra, Type I/heterotic duality and M-theory amplitudes, JHEP 12 (2016) 060 [arXiv: 1604.00324] [INSPIRE].

[101] M.B. Green and J.H. Schwarz, Supersymmetrical Dual String Theory. 2. Vertices and Trees, Nucl. Phys. B 198 (1982) 252 [INSPIRE].

[102] M.B. Green and J.H. Schwarz, Supersymmetrical Dual String Theory. 3. Loops and Renormalization, Nucl. Phys. B 198 (1982) 441 [INSPIRE].

[103] J.H. Schwarz, Superstring Theory, Phys. Rept. 89 (1982) 223 [INSPIRE].

[104] D.J. Gross and E. Witten, Superstring Modifications of Einstein's Equations, Nucl. Phys. B 277 (1986) 1 [INSPIRE].

[105] S. Sannan, Gravity as the Limit of the Type II Superstring Theory, Phys. Rev. D 34 (1986) 1749 [INSPIRE].

[106] A. Galperin, E. Ivanov, V. Ogievetsky and E. Sokatchev, Harmonic Supergraphs. Feynman Rules and Examples, Class. Quant. Grav. 2 (1985) 617 [INSPIRE].

[107] L. Mason and D. Skinner, Ambitwistor strings and the scattering equations, JHEP 07 (2014) 048 [arXiv: 1311.2564] [inSPIRE].

[108] T. Adamo, E. Casali and D. Skinner, A Worldsheet Theory for Supergravity, JHEP 02 (2015) 116 [arXiv: 1409.5656] [inSPIRE].

[109] F. Cachazo, S. He and E.Y. Yuan, Scattering of Massless Particles in Arbitrary Dimensions, Phys. Rev. Lett. 113 (2014) 171601 [arXiv:1307.2199] [InSPIRE].

[110] F. Cachazo, S. He and E.Y. Yuan, Scattering of Massless Particles: Scalars, Gluons and Gravitons, JHEP 07 (2014) 033 [arXiv:1309.0885] [INSPIRE].

[111] T. Adamo, E. Casali and D. Skinner, Ambitwistor strings and the scattering equations at one loop, JHEP 04 (2014) 104 [arXiv: 1312.3828] [INSPIRE].

[112] I. Bandos, Twistor/ambitwistor strings and null-superstrings in spacetime of $D=4,10$ and 11 dimensions, JHEP 09 (2014) 086 [arXiv: 1404.1299] [INSPIRE].

[113] I.A. Bandos, J.A. de Azcarraga and C. Miquel-Espanya, Superspace formulations of the (super)twistor string, JHEP 07 (2006) 005 [hep-th/0604037] [INSPIRE].

[114] N. Berkovits, An Alternative string theory in twistor space for $N=4$ superYang-Mills, Phys. Rev. Lett. 93 (2004) 011601 [hep-th/0402045] [INSPIRE].

[115] W. Siegel, Untwisting the twistor superstring, hep-th/0404255 [INSPIRE].

[116] Y. Geyer, A.E. Lipstein and L.J. Mason, Ambitwistor Strings in Four Dimensions, Phys. Rev. Lett. 113 (2014) 081602 [arXiv: 1404.6219] [INSPIRE].

[117] A. Lipstein and V. Schomerus, Towards a Worldsheet Description of $N=8$ Supergravity, arXiv: 1507.02936 [INSPIRE]. 
[118] L.V. Bork and A.I. Onishchenko, Four dimensional ambitwistor strings and form factors of local and Wilson line operators, Phys. Rev. D 97 (2018) 126013 [arXiv:1704.04758] [INSPIRE].

[119] J.A. Farrow and A.E. Lipstein, From $4 d$ Ambitwistor Strings to On Shell Diagrams and Back, JHEP 07 (2017) 114 [arXiv: 1705. 07087] [INSPIRE].

[120] E. Casali and P. Tourkine, On the null origin of the ambitwistor string, JHEP 11 (2016) 036 [arXiv: 1606. 05636] [INSPIRE].

[121] E. Casali, Y. Herfray and P. Tourkine, The complex null string, Galilean conformal algebra and scattering equations, JHEP 10 (2017) 164 [arXiv: 1707.09900] [INSPIRE].

[122] T. Adamo, E. Casali, L. Mason and S. Nekovar, Amplitudes on plane waves from ambitwistor strings, JHEP 11 (2017) 160 [arXiv:1708.09249] [INSPIRE]. 